\title{
Llull and Copeland Voting Computationally Resist Bribery and Constructive Control
}

\author{
Piotr Faliszewski \\ Department of Computer Science, AGH University of Science and Technology \\ Kraków, Poland
}

\section{Edith Hemaspaandra}

Department of Computer Science, Rochester Institute of Technology

Rochester, NY 14623 USA

Lane A. Hemaspaandra

Department of Computer Science, University of Rochester

Rochester, NY 14627 USA

Jörg Rothe

Institut für Informatik, Heinrich-Heine-Universität Düsseldorf

40225 Düsseldorf, Germany
FALISZEw@AGH.EDU.PL

EH@CS.RIT.EDU

LANE@CS.ROCHESTER.EDU

ROTHE@CS.UNI-DUESSELDORF.DE

\begin{abstract}
Control and bribery are settings in which an external agent seeks to influence the outcome of an election. Constructive control of elections refers to attempts by an agent to, via such actions as addition/deletion/partition of candidates or voters, ensure that a given candidate wins. Destructive control refers to attempts by an agent to, via the same actions, preclude a given candidate's victory. An election system in which an agent can sometimes affect the result and it can be determined in polynomial time on which inputs the agent can succeed is said to be vulnerable to the given type of control. An election system in which an agent can sometimes affect the result, yet in which it is NP-hard to recognize the inputs on which the agent can succeed, is said to be resistant to the given type of control.

Aside from election systems with an NP-hard winner problem, the only systems previously known to be resistant to all the standard control types were highly artificial election systems created by hybridization. This paper studies a parameterized version of Copeland voting, denoted by Copeland ${ }^{\alpha}$, where the parameter $\alpha$ is a rational number between 0 and 1 that specifies how ties are valued in the pairwise comparisons of candidates. In every previously studied constructive or destructive control scenario, we determine which of resistance or vulnerability holds for Copeland ${ }^{\alpha}$ for each rational $\alpha, 0 \leq \alpha \leq 1$. In particular, we prove that Copeland ${ }^{0.5}$, the system commonly referred to as "Copeland voting," provides full resistance to constructive control, and we prove the same for Copeland ${ }^{\alpha}$, for all rational $\alpha, 0<\alpha<1$. Among systems with a polynomial-time winner problem, Copeland voting is the first natural election system proven to have full resistance to constructive control. In addition, we prove that both Copeland ${ }^{0}$ and Copeland ${ }^{1}$ (interestingly, Copeland ${ }^{1}$ is an election system developed by the thirteenth-century mystic Llull) are resistant to all standard types of constructive control other than one variant of addition of candidates. Moreover, we show that for each rational $\alpha, 0 \leq \alpha \leq 1$, Copeland ${ }^{\alpha}$ voting is fully resistant to bribery attacks, and we establish fixed-parameter tractability of bounded-case control for Copeland ${ }^{\alpha}$.

We also study Copeland ${ }^{\alpha}$ elections under more flexible models such as microbribery and extended control, we integrate the potential irrationality of voter preferences into many of our results, and we prove our results in both the unique-winner model and the nonunique-winner model. Our vulnerability results for microbribery are proven via a novel technique involving min-cost network flow.
\end{abstract}




\section{Introduction}

This section gives some history and an outline of our results.

\subsection{Some Historical Remarks: Llull's and Copeland's Election Systems}

Elections have played an important role in human societies for thousands of years. For example, elections were of central importance in the democracy of ancient Athens. There citizens typically could only agree (vote yes) or disagree (vote no) with the speaker, and simple majority-rule was in effect. The mathematical study of elections, give or take a few discussions by the ancient Greeks and Romans, was until recently thought to have been initiated only a few hundred years ago, namely in the breakthrough work of Borda and Condorcet - later in part reinvented by Dodgson (see, e.g., McLean and Urken, 1995, for reprints of these classic papers). One of the most interesting results of this early work is Condorcet's (1785) observation that if one conducts elections with more than two alternatives then even if all voters have rational (i.e., transitive) preferences, the society in aggregate can be irrational (indeed, can have cycles of strict preference). Nonetheless, Condorcet believed that if there exists a candidate $c$ such that $c$ defeats each other candidate in head-to-head contests then $c$ should win the election (see, e.g., page 114 of McLean and Urken, 1995). Such a candidate is called a Condorcet winner. Clearly, there can be at most one Condorcet winner in any election, and there might be none.

This understanding of history has been reconsidered during the past few decades, as it has been discovered that the study of elections was considered deeply as early as the thirteenth century (see Hägele and Pukelsheim, 2001, and the citations therein regarding Ramon Llull and the fifteenthcentury figure Cusanus, especially the citations that in Hägele and Pukelsheim, 2001, are numbered 3, 5, and 24-27). Ramon Llull (b. 1232, d. 1315), a Catalan mystic, missionary, and philosopher developed an election system that (a) has an efficient winner-determination procedure and (b) elects a Condorcet winner whenever one exists and otherwise elects candidates that are, in a certain sense, closest to being Condorcet winners.

Llull's motivation for developing an election system was to obtain a method of choosing abbesses, abbots, bishops, and perhaps even the pope. His election ideas never gained public acceptance in medieval Europe and were long forgotten.

It is interesting to note that Llull allowed voters to have so-called irrational preferences. Given three candidates, $c, d$, and $e$, it was perfectly acceptable for a voter to prefer $c$ to $d, d$ to $e$, and $e$ to $c$. On the other hand, in modern studies of voting and election systems each voter's preferences are most typically modeled as a linear order over all candidates. (In this paper, as is common when discussing elections, "linear order" implies strictness, i.e., no tie in the ordering; that is, by "linear order" we mean a strict, complete order, i.e., an irreflexive, antisymmetric, complete, transitive relation.) Yet allowing irrationality is very tempting and natural. Consider Bob, who likes to eat out but is often in a hurry. Bob prefers diners to fast food because he is willing to wait a little longer to get better food. Also, given a choice between a fancy restaurant and a diner he prefers the fancy restaurant, again because he is willing to wait somewhat longer to get better quality. However, given the choice between a fast-food place and a fancy restaurant Bob might reason that he is not willing to wait so much longer to be served at the fancy restaurant and so will choose fast food instead. Thus regarding catering options, Bob's preferences are irrational in our sense, i.e., intransitive. When voters make their choices based on multiple criteria-a very common and natural occurrence both among humans and software agents—such irrationalities can 
occur. Another example where irrationality might naturally occur, as suggested by a referee, is the case when each voter is a delegate of some group (having an odd number of members), and between each pair of alternatives each delegate votes for whichever alternative a majority of his or her constituents prefers among that pair.

Llull's election system is remarkably similar to what is now known as "Copeland elections" (Copeland, 1951), a more than half-century old voting procedure that is based on pairwise comparisons of candidates: The winner (by a majority of votes-in this paper "majority" always, as is standard, means strict majority) of each such head-to-head contest is awarded one point and the loser is awarded zero points; in ties, both parties are (in the most common interpretation of Copeland's meaning) awarded half a point; whoever collects the most points over all these contests (including tie-related points) is the election's winner. In fact, the point value awarded for ties in such head-to-head majority-rule contests is treated in two ways in the literature when speaking of Copeland elections: half a point (most common) and zero points (less common). To provide a framework that can capture both those notions, as well as capturing Llull's system and the whole family of systems created by choices of how we value ties, we propose and introduce a parameterized version of Copeland elections, denoted by Copeland ${ }^{\alpha}$, where the parameter $\alpha$ is a rational number, $0 \leq \alpha \leq 1$, and in the case of a tie both candidates receive $\alpha$ points. So the system widely referred to in the literature as "Copeland elections" is Copeland ${ }^{0.5}$, where tied candidates receive half a point each (see, e.g., Saari and Merlin, 1996, and Merlin and Saari, 1997; the definition used by Conitzer, Sandholm, \& Lang, 2007, can be scaled to be equivalent to Copeland ${ }^{0.5}$ ). Copeland ${ }^{0}$, where tied candidates come away empty-handed, has sometimes also been referred to as "Copeland elections" (see, e.g., Procaccia, Rosenschein, and Kaminka, 2007, and Faliszewski, Hemaspaandra, Hemaspaandra, and Rothe, 2007, an early version of this paper). The above-mentioned election system proposed by Ramon Llull in the thirteenth century is in this notation Copeland ${ }^{1}$, where tied candidates are awarded one point each, just like winners of head-to-head contests. ${ }^{1}$ The group stage of the FIFA World Cup finals is in essence a collection of Copeland ${ }^{\frac{1}{3}}$ tournaments.

At first glance, one might be tempted to think that the definitional perturbation due to the parameter $\alpha$ in Copeland ${ }^{\alpha}$ elections is negligible. However, it in fact can make the dynamics of Llull's system quite different from those of, for instance, Copeland ${ }^{0.5}$ or Copeland ${ }^{0}$. Specific examples witnessing this claim, both regarding complexity results and regarding their proofs, are given at the end of Section 1.3.

Finally, we mention that a probabilistic variant of Copeland voting (known as the Jech method) was defined already in 1929 by Zermelo (1929) and later on was reintroduced by several other researches (see, e.g., Levin and Nalebuff, 1995, for further references and a description of the Jech method). We note in passing that the Jech method is applicable even when it is fed incomplete information. In the present paper, however, we do not consider incomplete-information or probabilistic scenarios, although we commend such settings as interesting for future work.

1. Page 23 of Hägele and Pukelsheim 2001 indicates in a way we find deeply convincing (namely by a direct quote of Llull's in-this-case-very-clear words from his Artifitium Electionis Personarum-which was rediscovered by those authors in the year 2000) that at least one of Llull's election systems was Copeland ${ }^{1}$, and so in this paper we refer to the both-candidates-score-a-point-on-a-tie variant as Llull voting.

In some settings Llull required the candidate and voter sets to be identical and had an elaborate two-stage tiehandling rule ending in randomization. We disregard these issues here and cast his system into the modern idiom for election systems. (However, we note in passing that there do exist some modern papers in which the voter and candidate sets are taken to be identical, see for example the work of and references in Altman and Tennenholtz, 2007.) 


\subsection{Computational Social Choice}

In general it is impossible to design a perfect election system. Arrow (1963) famously showed that there is no social choice system that satisfies a certain small set of arguably reasonable requirements, and later Gibbard (1973), Satterthwaite (1975), and Duggan and Schwartz (2000) showed that any natural election system can sometimes be manipulated by strategic voting, i.e., by a voter revealing different preferences than his or her true ones in order to affect an election's result in his or her favor. Also, no natural election system with a polynomial-time winner-determination procedure has yet been shown to be resistant to all types of control via procedural changes. Control refers to attempts by an external agent (called "the chair") to, via such actions as addition/deletion/partition of candidates or voters, make a given candidate win the election (in the case of constructive control, Bartholdi, Tovey, and Trick, 1992) or preclude a given candidate's victory (in the case of destructive control, Hemaspaandra, Hemaspaandra, and Rothe, 2007a).

These obstacles are very discouraging, but the field of computational social choice theory grew in part from the realization that computational complexity provides a potential shield against manipulation/control/etc. In particular, around 1990, Bartholdi, Tovey, and Trick (1989a), Bartholdi and Orlin (1991), and Bartholdi et al. (1992) brilliantly observed that while we perhaps might not be able to make manipulation (i.e., strategic voting) and control of elections impossible, we could at least try to make such manipulation and control so computationally difficult that neither voters nor election organizers will attempt it. For example, if there is a way for a committee's chair to set up an election within the committee in such a way that his or her favorite option is guaranteed to win, but the chair's computational task would take a million years, then for all practical purposes we may feel that the chair is prevented from finding such a set-up.

Since the seminal work of Bartholdi, Orlin, Tovey, and Trick, a large body of research has been dedicated to the study of computational properties of election systems. Some topics that have received much attention are the complexity of manipulating elections (Conitzer \& Sandholm, 2003, 2006; Conitzer et al., 2007; Elkind \& Lipmaa, 2005; Hemaspaandra \& Hemaspaandra, 2007; Procaccia \& Rosenschein, 2007; Meir, Procaccia, Rosenschein, \& Zohar, 2008) and of controlling elections via procedural changes (Hemaspaandra et al., 2007a; Hemaspaandra, Hemaspaandra, \& Rothe, 2007b; Meir et al., 2008; Erdélyi, Nowak, \& Rothe, 2008b). Recently, Faliszewski, Hemaspaandra, and Hemaspaandra (2006a) introduced the study of the complexity of bribery in elections. Bribery shares some features of manipulation and some features of control. In particular, the briber picks the voters he or she wants to affect (as in voter control problems) and asks them to vote as he or she wishes (as in manipulation). (For additional citations and pointers, see the recent survey Faliszewski, Hemaspaandra, Hemaspaandra, and Rothe, 2009.)

In this paper we study Copeland ${ }^{\alpha}$ elections with respect to the computational complexity of bribery and procedural control; see Faliszewski, Hemaspaandra, and Schnoor 2008 for a study of manipulation within Copeland ${ }^{\alpha}$.

The study of election systems and their computational properties, such as the complexity of their manipulation, control, and bribery problems, is an important topic in multiagent systems. Agents/voters may have different, often conflicting, individual preferences over the given alternatives (or candidates) and voting rules (or, synonymously, election systems) provide a useful method for agents to come to a "reasonable" decision on which alternative to choose. Thus elections can be employed in multiagent settings and also in other contexts to solve many practical problems. As just a few examples, we mention that Ephrati and Rosenschein (1997) use elections for planning, Ghosh, 
Mundhe, Hernandez, and Sen (1999) develop a recommender system for movies that is based on voting, and Dwork, Kumar, Naor, and Sivakumar (2001) use elections to aggregate results from multiple web-search engines. In a multiagent setting we may have hundreds of elections happening every minute and we cannot hope to carefully check in each case whether the party that organized the election attempted some procedural change to skew the results. However, if it is computationally hard to find such procedural changes then we can hope it is practically infeasible for the organizers to undertake them.

A standard technique for showing that a particular election-related problem (for example, the problem of deciding whether the chair can make his or her favorite candidate a winner by influencing at most $k$ voters not to cast their votes) is computationally intractable is to show that it is NP-hard. This approach is taken in almost all the papers on computational social choice cited above, and it is the approach that we take in this paper. One of the justifications for using NP-hardness as a barrier against manipulation and control of elections is that in multiagent settings any attempts to influence the election's outcome are made by computationally bounded software agents that have neither human intuition nor the computational ability to solve NP-hard problems.

Recently, Conitzer and Sandholm (2006), Procaccia and Rosenschein (2007), Homan and Hemaspaandra (to appear), and McCabe-Dansted, Pritchard, and Slinko (2008) have studied the frequency (or sometimes, probability weight) of correctness of heuristics for voting problems. Although this is a fascinating and important direction, it does not at this point remove the need to study worst-case hardness. Indeed, we view worst-case study as a natural prerequisite to a frequency-ofhardness attack: After all, there is no point in seeking frequency-of-hardness results if the problem at hand is in $\mathrm{P}$ to begin with. And if one cannot even prove worst-case hardness for a problem, then proving "average-case" hardness is even more beyond reach. Also, current frequency results have debilitating limitations (for example, being locked into specific distributions; depending on unproven assumptions; and adopting "tractability" notions that declare undecidable problems tractable and that are not robust under even linear-time reductions). These models are arguably not ready for prime time and, contrary to some people's impression, do not imply (and do not have the goal of implying, since they are studying frequency of hardness) average-case polynomial runtime claims. Erdélyi, Hemaspaandra, Rothe, and Spakowski (2007) and Homan and Hemaspaandra (to appear) provide discussions of some of these issues. Regarding the recent work of Friedgut, Kalai, and Nisan (2008) (see also Xia and Conitzer, 2008a, 2008b), that very interesting work is not on control, and the lower bounds proven there do not show that one can manipulate most of the time, but rather that work provides lower bounds that unfortunately go to zero as the number of voters increases, for the case there studied. Of course, the limitations of current results on frequency of hardness surely do not mean that the direction is not interesting; clearly, the field should do its best to go beyond those limitations.

\subsection{Outline of Our Results}

The goal of this paper is to study Copeland ${ }^{\alpha}$ elections from the point of view of computational social choice theory, in the setting where voters are rational and in the setting where voters are allowed to have irrational preferences. (Note: When we henceforward say "irrational voters," we mean that the voters may have irrational preferences, not that they each must.) We study the issues of bribery and control and we point the reader to Faliszewski et al. 2008 for work on manipulation. (Very briefly summarized, the work of Faliszewski et al., 2008, on manipulation of Copeland ${ }^{\alpha}$ elections 
shows that for all rational $\alpha, 0<\alpha<1, \alpha \neq \frac{1}{2}$, the coalitional manipulation problem in unweighted Copeland ${ }^{\alpha}$ elections, even for coalitions of just two manipulators, is NP-complete. Some of the constructions of the present paper have been adopted or adapted in that paper in order to prove results about manipulation.)

Bribery and control problems have some very natural real-life interpretations. For example, during presidential elections a candidate might want to encourage as many of his or her supporters as possible to vote ("get-out-the-vote" efforts): control via addition of voters; elections can be held at an inconvenient date for a group of voters (e.g., a holiday) or at a hard-to-reach location (e.g., requiring one to own a car, or such that getting to the location involves passing dangerous areas): control via deleting voters; one can choose voting districts in a way favorable to a particular candidate or party (gerrymandering): control via partitioning voters; one can introduce a new candidate to the election in the hope that he or she will steal votes away from the opponents of one's favorite candidate without affecting the favorite candidate's performance: control via adding candidates. All the other control scenarios that we study also have natural interpretations.

Similarly, bribery is a natural and important issue in the context of elections. We stress, however, that bribery problems do not necessarily need to correspond to cheating or any sort of illegal action. One could view bribery problems as, for example, problems of finding the minimum number of voters who can switch the result of the election and, thus, as problems of finding coalitions, especially if one assigns prices to voters to measure the difficulty of convincing a particular voter to join the coalition (see, e.g., Faliszewski, 2008, for an example of a bribery problem where such an interpretation is very natural).

It is quite natural to study control and bribery both in constructive settings (where we want to make our favorite candidate a winner) and in destructive settings (where we try to prevent a candidate from winning). In the context of real-life elections, one often hears voters speaking of which candidate they hope will win, but one also often hears voters expressing the sentiment "Anyone but him." The constructive and destructive settings correspond to actions that agents belonging to these groups might be interested in.

One of the main achievements of this paper is to classify which of resistance or vulnerability holds for Copeland ${ }^{\alpha}$ in every previously studied control scenario for each rational value of $\alpha$, $0 \leq \alpha \leq 1$. In doing so, we provide an example of a control problem where the complexity of Copeland $^{0.5}$ (which is the system commonly referred to as "Copeland") differs from that of both Copeland $^{0}$ and Copeland ${ }^{1}$ : While the latter two problems are vulnerable to constructive control by adding (an unlimited number of) candidates, Copeland ${ }^{0.5}$ is resistant to this control type (see Section 2 for definitions and Theorem 4.10 for this result).

In fact, Copeland (i.e., Copeland ${ }^{0.5}$ ) is the first natural election system (with a polynomial-time winner problem) proven to be resistant to every type of constructive control that has been proposed in the literature to date. This result closes a 15 -year quest for a natural election system fully resistant to constructive control. ${ }^{2}$

2. A referee wondered whether (and speculated that) virtually every common rule (other than plurality and Condorcet, said the referee, although actually plurality displays breathtakingly many resistances itself, albeit not all the constructive resistances) would display just as broad resistance to control as does Copeland, were one to obtain results for those rules. This of course is an open issue, but we see no reason to think it will be the case (and approval voting already provides a counterexample, see Hemaspaandra et al., 2007a). And even if that were the case and most other rules resisted as many control types, we suspect that the pattern of which types are resisted will differ among the rules, although it is the case that the four "quadrants" (of constructive/destructive and voter/candidate do seem to often stand or fall as a block). That pattern seems to us something that is of natural importance, since one's choice 
We also show that Copeland ${ }^{\alpha}$ is resistant to both constructive and destructive bribery, for both the case of rational voters and the case of irrational voters. Our hardness proofs work for the case of unweighted voters without price tags (see Faliszewski et al., 2006a) and thus, naturally, apply as well to the more involved scenarios of weighted unpriced voters, unweighted priced voters, and weighted priced voters.

To prove our bribery results, we introduce a method of controlling the relative performances of certain voters in such a way that, if one sets up other restrictions appropriately, the legal possibilities for bribery actions are sharply constrained. We call our approach "the UV technique," since it is based on dummy candidates $u$ and $v$. The proofs of Theorems 3.2 and 3.4 are particular applications of this method. We feel that the UV technique will be useful, even beyond the scope of this paper, for the analysis of bribery in other election systems based on head-to-head contests.

We also study Copeland ${ }^{\alpha}$ elections under more flexible models such as "microbribery" (see Section 3.2) and "extended control" (see Section 4.3). We show that Copeland ${ }^{\alpha}$ (with irrational voters allowed) is vulnerable to destructive microbribery and to destructive candidate control via providing fairly simple greedy algorithms. In contrast, our polynomial-time algorithms for constructive microbribery are proven via a technique involving min-cost network flows. To the best of our knowledge, this is the first application of min-cost flows to election problems. We believe that the range of applicability of flow networks to election problems extends well beyond microbribery for Copeland ${ }^{\alpha}$ elections and we point the reader to a recent, independent paper by Procaccia, Rosenschein, and Zohar $(2008)^{3}$ and to a paper by Faliszewski (2008) for examples of such applications.

We also mention that during our study of Copeland control we noticed that the proof of an important result of Bartholdi et al. (1992, Theorem 12), namely, that Condorcet voting is resistant to constructive control by deleting voters, is invalid. The invalidity is due to the proof centrally using nonstrict voters, in violation of Bartholdi et al.'s (1992) (and our) model, and the invalidity seems potentially daunting or impossible to fix with the proof approach taken there. We note also that Theorem 14 of the same paper has a similar flaw. In Section 5 we validly reprove their claimed results using our techniques.

As mentioned in Section 1.1, Copeland ${ }^{\alpha}$ elections may behave quite differently depending on the value of the tie-rewarding parameter $\alpha$. We now give concrete examples to make this case. Specifically, proofs of results for Copeland ${ }^{\alpha}$ occasionally differ considerably for distinct values of $\alpha$, and in some cases even the computational complexity of various control and manipulation problems (for the manipulation case see Faliszewski et al., 2008) may jump between P membership and NP-completeness depending on $\alpha$. Regarding control, we have already noted that Theorem 4.10 shows that some control problem (namely, control by adding an unlimited number of candidates) for Copeland ${ }^{\alpha}$ is NP-complete for each rational $\alpha$ with $0<\alpha<1$, yet Theorem 4.11 shows that same control problem to be in $\mathrm{P}$ for $\alpha \in\{0,1\}$. To give another example involving a different

of election rule should probably (along with many other factors that should influence rule choice) be shaped by the strength of the rule with respect to resisting the types of attacks one expects the system to be faced with. For example, Copeland is exceedingly strong — in fact, perfect—with respect to the constructive control types studied here. In contrast, plurality, Condorcet, and approval are not (Bartholdi et al., 1992; Hemaspaandra et al., 2007a), and we can't speak to the issue of as yet unstudied systems. And as to what holds for other rules, we suspect that the dream-case path would be to find broad results that in one stroke reveal the control-resistance patterns of whole classes of election systems. For example, see Hemaspaandra and Hemaspaandra 2007, which does essentially that for manipulation of scoring systems.

3. Procaccia et al. (2008) independently of our work in Faliszewski et al. 2007 used a similar technique in their work regarding the complexity of achieving proportional representation. 
control problem, namely control by partition of candidates with the ties-eliminate tie-handling rule (see Section 2), we note that the proofs of Theorem 4.15 (which applies to $\alpha=1$ for this control problem within Copeland ${ }^{\alpha}$ ) and of Theorem 4.16 (which applies to all rational $\alpha$ with $0 \leq \alpha<1$ for the same problem) differ substantially. Regarding constructive microbribery, the vulnerability constructions for $\alpha=0$ (see Lemma 3.13) and $\alpha=1$ (see Lemma 3.15) significantly differ from each other, and neither of them works for tie-rewarding values other than 0 and 1 . The above remarks notwithstanding, for most of our results we show that it is possible to obtain a unifiedthough due to this uniformity sometimes rather involved-construction that works for Copeland ${ }^{\alpha}$ for every rational $\alpha, 0 \leq \alpha \leq 1$.

\subsection{Organization}

This paper is organized as follows. In Section 2, we formalize the notion of elections and in particular of Copeland ${ }^{\alpha}$ elections, we introduce some useful notation, and we formally define the control and bribery problems we are interested in. In Section 3, we show that for each rational $\alpha, 0 \leq \alpha \leq 1$, Copeland ${ }^{\alpha}$ elections are fully resistant to bribery, both in the case of rational voters and in the case of irrational voters. On the other hand, if one changes the bribery model to allow "microbribes" of voters (a fine-grained approach to bribery, in which the more one changes a voter's vote, the more one has to pay the voter), we prove vulnerability for each rational $\alpha, 0 \leq \alpha \leq 1$, in the irrationalvoters destructive case and for some specific values of $\alpha$ in the irrational-voters constructive case. In Sections 4.1 and 4.2, we present our results on procedural control for Copeland ${ }^{\alpha}$ elections for each rational $\alpha$ with $0 \leq \alpha \leq 1$. We will see that very broad resistance holds for the constructive-control cases. Section 4.3 presents our results on fixed-parameter tractability of bounded-case control for Copeland ${ }^{\alpha}$. Section 5 provides valid proofs for several control problems for Condorcet elections (studied by Bartholdi et al., 1992) whose original proofs were invalid due to being at odds with the model of elections used in Bartholdi et al. 1992. We conclude the paper with a brief summary in Section 6 and by stating some open problems.

If every proof were included in this paper, it would be extremely long and difficult to read. Nonetheless, it is of course important to make proofs available for our claims. We have handled this as follows. We have made available as Faliszewski, Hemaspaandra, Hemaspaandra, and Rothe 2008b a full technical report version of this paper, with complete and detailed proofs of essentially every result. And in the current article, for proofs that would be repetitive or tedious relative to other proofs that we do include here, we simply have not included those proofs here and have instead included in their place a pointer to the detailed proof of the result in the full technical report version.

\section{Preliminaries}

This section defines many of the notions we use in this paper, such as various election systems, election problems, and hardness notions.

\subsection{Elections: The Systems of Llull and Copeland}

An election $E=(C, V)$ consists of a finite candidate set $C=\left\{c_{1}, \ldots, c_{m}\right\}$ and a finite collection $V$ of voters, where each voter is represented (individually, except later when we discuss succinct inputs) 
via his or her preferences over the candidates. An election system (or an election rule) is a rule that determines the winner(s) of each given election, i.e., a mapping from pairs $(C, V)$ to subsets of $C$.

We consider two ways in which voters can express their preferences. In the rational case (our default case), each voter's preferences are represented as a linear order over the set $C$, ${ }^{4}$ i.e., each voter $v_{i}$ has a preference list $c_{i_{1}}>c_{i_{2}}>\cdots>c_{i_{m}}$, with $\left\{i_{1}, i_{2}, \ldots, i_{m}\right\}=\{1,2, \ldots, m\}$. In the irrational case, each voter's preferences are represented as a preference table that for every unordered pair of distinct candidates $c_{i}$ and $c_{j}$ in $C$ indicates whether the voter prefers $c_{i}$ to $c_{j}$ (i.e., $c_{i}>c_{j}$ ) or prefers $c_{j}$ to $c_{i}$ (i.e., $c_{j}>c_{i}$ ).

Some well-known election rules for the case of rational voters are plurality, Borda count, and Condorcet. Plurality elects the candidate(s) that are ranked first by the largest number of voters. Borda count elects the candidate(s) that receive the most points, where each voter $v_{i}$ gives each candidate $c_{j}$ as many points as the number of candidates $c_{j}$ is preferred to with respect to $v_{i}$ 's preferences. A candidate $c_{i}$ is a Condorcet winner if for every other candidate $c_{j}$ it holds that $c_{i}$ is preferred to $c_{j}$ by a majority of voters. Note that each election instance will have at most one Condorcet winner.

In this paper, we introduce a parameterized version of Copeland's (1951) election system, which we denote by Copeland ${ }^{\alpha}$, where the parameter $\alpha$ is a rational number between 0 and 1 that specifies how ties are rewarded in the head-to-head majority-rule contests between any two distinct candidates.

Definition 2.1 Let $\alpha, 0 \leq \alpha \leq 1$, be a rational number. In a Copeland ${ }^{\alpha}$ election, for each head-tohead contest between two distinct candidates, if some candidate is preferred by a majority of voters then he or she obtains one point and the other candidate obtains zero points, and if a tie occurs then both candidates obtain $\alpha$ points. Let $E=(C, V)$ be an election. For each $c \in C$, $\operatorname{score}_{E}^{\alpha}(c)$ is (by definition) the sum of c's Copeland ${ }^{\alpha}$ points in E. Every candidate $c$ with maximum $\operatorname{score}_{E}^{\alpha}(c)$ (i.e., every candidate c satisfying $\left.(\forall d \in C)\left[\operatorname{score}_{E}^{\alpha}(c) \geq \operatorname{score}_{E}^{\alpha}(d)\right]\right)$ wins.

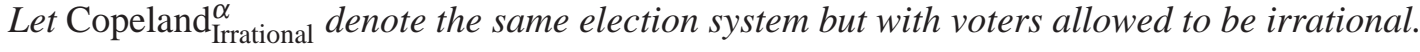

As mentioned earlier, in the literature the term "Copeland elections" is most often used for the system Copeland $^{0.5}$ (e.g., Saari and Merlin, 1996, Merlin and Saari, 1997, and a rescaled version of Conitzer et al., 2007), but has occasionally been used for Copeland ${ }^{0}$ (e.g., Procaccia et al., 2007, and Faliszewski et al., 2007, an early version of this paper). As mentioned earlier, the system Copeland ${ }^{1}$ was proposed by Llull in the thirteenth century (see the literature pointers given in the introduction) and so is called Llull voting.

We now define some notation to help in the discussion of Copeland ${ }^{\alpha}$ elections. Informally put, if $E=(C, V)$ is an election and if $c_{i}$ and $c_{j}$ are any two candidates in $C$ then by $\operatorname{vs}_{E}\left(c_{i}, c_{j}\right)$ we mean the surplus of votes that candidate $c_{i}$ has over $c_{j}$. Formally, we define this notion as follows.

Definition 2.2 Let $E=(C, V)$ be an election and let $c_{i}$ and $c_{j}$ be two arbitrary candidates from $C$. Define the relative vote-score of $c_{i}$ with respect to $c_{j}$ by

$$
\operatorname{vs}_{E}\left(c_{i}, c_{j}\right)= \begin{cases}0 & \text { if } c_{i}=c_{j} \\ \|\left\{v \in V \mid v \text { prefers } c_{i} \text { to } c_{j}\right\}\|-\|\left\{v \in V \mid v \text { prefers } c_{j} \text { to } c_{i}\right\} \| & \text { otherwise } .\end{cases}
$$

4. In this paper, we take "linear order" to mean a strict total order. This is a common convention within voting theory, see, e.g., the book Austen-Smith and Banks 2000. However, we mention that in the field of mathematics the term "linear order" is typically taken to allow nonstrictness, i.e., to allow ties. 
So, if $c_{i}$ defeats $c_{j}$ in a head-to-head contest in $E$ then $\operatorname{vs}_{E}\left(c_{i}, c_{j}\right)>0$, if they are tied then $\operatorname{vs}_{E}\left(c_{i}, c_{j}\right)=0$, and if $c_{j}$ defeats $c_{i}$ then $\operatorname{vs}_{E}\left(c_{i}, c_{j}\right)<0$. (Throughout this paper, "defeats" excludes the possibility of a tie, i.e., "defeats" means "(strictly) defeats." We will say "ties-or-defeats" when we wish to allow a tie to suffice.) Clearly, $\operatorname{vs}_{E}\left(c_{i}, c_{j}\right)=-\mathrm{vs}_{E}\left(c_{j}, c_{i}\right)$. We often speak, in the plural, of relative vote-scores when we mean a group of results of head-to-head contests between particular candidates.

Let $\alpha, 0 \leq \alpha \leq 1$, be a rational number. Definition 2.1 introduced $\operatorname{score}_{E}^{\alpha}(c)$, the Copeland ${ }^{\alpha}$ score of candidate $c$ in election $E$. Note that for each candidate $c_{i} \in C$,

$$
\begin{aligned}
\operatorname{score}_{E}^{\alpha}\left(c_{i}\right)= & \|\left\{c_{j} \in C \mid c_{i} \neq c_{j} \text { and } \operatorname{vs}_{E}\left(c_{i}, c_{j}\right)>0\right\} \| \\
& +\alpha \|\left\{c_{j} \in C \mid c_{i} \neq c_{j} \text { and } \operatorname{vs}_{E}\left(c_{i}, c_{j}\right)=0\right\} \| .
\end{aligned}
$$

In particular, we have $\operatorname{score}_{E}^{0}\left(c_{i}\right)=\|\left\{c_{j} \in C \mid c_{i} \neq c_{j}\right.$ and $\left.\operatorname{vs}_{E}\left(c_{i}, c_{j}\right)>0\right\} \|$, and $\operatorname{score}_{E}^{1}\left(c_{i}\right)=\|\left\{c_{j} \in\right.$ $C \mid c_{i} \neq c_{j}$ and $\left.\operatorname{vs}_{E}\left(c_{i}, c_{j}\right) \geq 0\right\} \|$. Note further that the highest possible Copeland ${ }^{\alpha}$ score in any election $E=(C, V)$ is $\|C\|-1$.

Recall that a candidate $c_{i} \in C$ is a Copeland ${ }^{\alpha}$ winner of $E=(C, V)$ if for all $c_{j} \in C$ it holds that $\operatorname{score}_{E}^{\alpha}\left(c_{i}\right) \geq \operatorname{score}_{E}^{\alpha}\left(c_{j}\right)$. (Clearly, some elections can have more than one Copeland ${ }^{\alpha}$ winner.) A candidate $c_{i}$ is a Condorcet winner of $E$ if $\operatorname{score}_{E}^{0}\left(c_{i}\right)=\|C\|-1$, that is, if $c_{i}$ defeats all other candidates in head-to-head contests.

In many of our constructions to be presented in the upcoming proofs, we use the following notation for rational voters.

Notation 2.3 Within every election we fix some arbitrary order over the candidates. Any occurrence of a subset $D$ of candidates in a preference list means the candidates from $D$ are listed with respect to that fixed order. Occurrences of $\overleftarrow{D}$ mean the same except that the candidates from $D$ are listed in the reverse order.

For example, if $C=\{a, b, c, d, e\}$, with the alphabetical order being used, and $D=\{a, c, e\}$ then $b>D>d$ means $b>a>c>e>d$, and $b>\overleftarrow{D}>d$ means $b>e>c>a>d$.

\subsection{Bribery and Control Problems}

We now describe the computational problems that we study in this paper. Our problems come in two flavors: constructive and destructive. In the constructive version the goal is to determine whether, via the bribery or control action type under study, it is possible to make a given candidate a winner of the election. In the destructive case the goal is to determine whether it is possible to prevent a given candidate from being a winner of the election.

Let $\mathscr{E}$ be an election system. In our case, $\mathscr{E}$ will be either Copeland ${ }^{\alpha}$ or Copeland ${ }_{\text {Irrational }}^{\alpha}$, where $\alpha, 0 \leq \alpha \leq 1$, is a rational number. The bribery problem for $\mathscr{E}$ with rational voters is defined as follows (Faliszewski et al., 2006a).

Name: $\mathscr{E}$-bribery and $\mathscr{E}$-destructive-bribery.

Given: A set $C$ of candidates, a collection $V$ of voters specified via their preference lists over $C$, a distinguished candidate $p \in C$, and a nonnegative integer $k$.

Question (constructive): Is it possible to make $p$ a winner of the $\mathscr{E}$ election resulting from $(C, V)$ by modifying the preference lists of at most $k$ voters? 
Question (destructive): Is it possible to ensure that $p$ is not a winner of the $\mathscr{E}$ election resulting from $(C, V)$ by modifying the preference lists of at most $k$ voters?

The version of this problem for elections with irrational voters allowed is defined exactly like the rational one, with the only difference being that voters are represented via preference tables rather than preference lists, and the briber may completely change a voter's preference table at unit cost. At the end of the present section, Section 2.2, we will describe the variants based on seeking to make $p$ be (or to preclude $p$ from being) a unique winner. Later in the paper we will study another variant of bribery problems - a variant in which one is allowed to perform microbribes: bribes for which the cost depends on each preference-table entry change, and the briber pays separately for each such change.

Bribery problems seek to change the outcome of elections via modifying the reported preferences of some of the voters. In contrast, control problems seek to change the outcome of an election by modifying the election's structure via adding/deleting/partitioning either candidates or voters. When formally defining these control types, we use the following naming conventions for the corresponding control problems. The name of a control problem starts with the election system used (when clear from context, it may be omitted), followed by CC for "constructive control" or by DC for "destructive control," followed by the acronym of the type of control: AC for "adding (a limited number of) candidates," $\mathrm{AC}_{\mathrm{u}}$ for "adding (an unlimited number of) candidates," DC for "deleting candidates," PC for "partition of candidates," RPC for "run-off partition of candidates," AV for "adding voters," DV for "deleting voters," and PV for "partition of voters." All the partitioning cases (PC, RPC, and PV) are two-stage elections, and we here use both tie-handling rules of Hemaspaandra et al. (2007a) for first-stage subelections in these two-stage elections. In particular, for all the partitioning cases, the acronym PC, RPC, and PV, respectively, is followed by the acronym of the tie-handling rule used in first-stage subelections, namely TP for "ties promote" (i.e., all winners of first-stage subelections are promoted to the final round of the election) and TE for "ties eliminate" (i.e., only unique winners of first-stage subelections are promoted to the final round of the election, so if there is more than one winner in a given first-stage subelection or there is no winner in a given first-stage subelection then that subelection does not move any of its candidates forward).

We now formally define our control problems. These definitions are due to Bartholdi et al. (1992) for constructive control and to Hemaspaandra et al. (2007a) for destructive control.

Let $\mathscr{E}$ be an election system. Again, $\mathscr{E}$ will here be either Copeland ${ }^{\alpha}$ or Copeland Irrational $^{\alpha}$, where $\alpha, 0 \leq \alpha \leq 1$, is a rational number. We describe our control problems as if they were for the case of rational preferences, but the irrational cases are perfectly analogous, except for replacing preference lists with preference tables.

\section{Control via Adding Candidates}

We start with two versions of control via adding candidates. In the unlimited version the goal of the election chair is to introduce candidates from a pool of spoiler candidates so as to make his or her favorite candidate a winner of the election (in the constructive case) or prevent his or her despised candidate from being a winner (in the destructive case). As suggested by the name of the problem, in the unlimited version the chair can introduce any subset of the spoiler candidates (none, some, or all are all legal options) into the election.

Name: $\mathscr{E}-\mathrm{CCAC}_{\mathrm{u}}$ and $\mathscr{E}-\mathrm{DCAC}_{\mathrm{u}}$ (control via adding an unlimited number of candidates). 
Given: Disjoint sets $C$ and $D$ of candidates, a collection $V$ of voters specified via their preference lists over the candidates in the set $C \cup D$, and a distinguished candidate $p \in C$.

Question $\left(\mathscr{E}-\mathrm{CCAC}_{\mathrm{u}}\right)$ : Is there a subset $E$ of $D$ such that $p$ is a winner of the $\mathscr{E}$ election with voters $V$ and candidates $C \cup E$ ?

Question $\left(\mathscr{E}-\mathrm{DCAC}_{\mathrm{u}}\right)$ : Is there a subset $E$ of $D$ such that $p$ is not a winner of the $\mathscr{E}$ election with voters $V$ and candidates $C \cup E$ ?

The definition of $\mathscr{E}-\mathrm{CCAC}_{\mathrm{u}}$ was (using different notation) introduced by Bartholdi et al. (1992). In contrast with the other control problems involving adding or deleting candidates or voters, in the adding candidates problem Bartholdi, Tovey, and Trick did not introduce a nonnegative integer $k$ that bounds the number of candidates (from the set $D$ ) the chair is allowed to add. We feel this asymmetry in their definitions is not well justified, ${ }^{5}$ and thus we define a with-change-parameter version of the control-by-adding-candidates problems, which we denote by $\mathrm{AC}_{1}$ (where the "l" stands for the fact that part of the problem instance is a limit on the number of candidates that can be added, in contrast with the model of Bartholdi et al., 1992, which we denote by $\mathrm{AC}_{\mathrm{u}}$ with the "u" standing for the fact that the number of added candidates is $u$ nlimited, at least in the sense of not being limited via a separately input integer). The with-parameter version is the long-studied case for $\mathrm{AV}, \mathrm{DV}$, and $\mathrm{DC}$, and we in this paper will use $\mathrm{AC}$ as being synonymous with $\mathrm{AC}_{1}$, and will thus use the notation $\mathrm{AC}$ for the rest of this paper when speaking of $\mathrm{AC}_{1}$. We suggest this as a natural regularization of the definitions and we hope this version will become the "normal" version of the adding-candidates problem for further study. However, we caution the reader that in earlier papers $\mathrm{AC}$ is used to mean $\mathrm{AC}_{\mathrm{u}}$.

In the present paper, we will obtain results not just for $\mathrm{AC}_{1}$ but also for the $\mathrm{AC}_{\mathrm{u}}$ case, in order to allow comparisons between the results of this paper and those of earlier works.

Turning now to what we mean by $\mathrm{AC}$ (equivalently, $\mathrm{AC}_{1}$ ), as per the above definition in $\mathscr{E}$-CCAC (i.e., $\mathscr{E}-\mathrm{CCAC}_{1}$ ) we ask whether it is possible to make the distinguished candidate $p$ a winner of some $\mathscr{E}$ election obtained by adding at most $k$ candidates from the spoiler candidate set $D$. (Note that $k$ is part of the input.) We define the destructive version, $\mathscr{E}$-DCAC (i.e., $\mathscr{E}$-DCAC ), analogously.

Name: $\mathscr{E}$-CCAC and $\mathscr{E}$-DCAC (control via adding a limited number of candidates).

Given: Disjoint sets $C$ and $D$ of candidates, a collection $V$ of voters specified via their preference lists over the candidates in the set $C \cup D$, a distinguished candidate $p \in C$, and a nonnegative integer $k$.

Question $(\mathscr{E}-\mathrm{CCAC})$ : Is there a subset $E$ of $D$ such that $\|E\| \leq k$ and $p$ is a winner of the $\mathscr{E}$ election with voters $V$ and candidates $C \cup E$ ?

Question $(\mathscr{E}$-DCAC): Is there a subset $E$ of $D$ such that $\|E\| \leq k$ and $p$ is not a winner of the $\mathscr{E}$ election with voters $V$ and candidates $C \cup E$ ?

5. Bartholdi et al. (1992) are aware of this asymmetry. They write: "To a certain extent the exact formalization of a problem is a matter of taste. [...] we could equally well have formalized [the problem of control via adding candidates] to be whether there are $K$ or fewer candidates to be added [...] It does not much matter for the problems we discuss, since both versions are of the same complexity" (Bartholdi et al., 1992). In contrast, the complexity of the problems studied here crucially hinges on which formalization is used, and we thus define both versions formally. 


\section{Control via Deleting CANDidates}

In constructive control via deleting candidates, the chair seeks to ensure that his or her favorite candidate $p$ is a winner of the election by suppressing at most $k$ candidates. In the destructive variant of this problem, the chair's goal is to block $p$ from winning by suppressing at most $k$ candidates other than $p .^{6}$

Name: $\mathscr{E}$-CCDC and $\mathscr{E}$-DCDC (control via deleting candidates).

Given: A set $C$ of candidates, a collection $V$ of voters represented via preference lists over $C$, a distinguished candidate $p \in C$, and a nonnegative integer $k$.

Question ( $\mathscr{E}-\mathrm{CCDC})$ : Is it possible to by deleting at most $k$ candidates ensure that $p$ is a winner of the resulting $\mathscr{E}$ election?

Question ( $\mathscr{E}$-DCDC): Is it possible to by deleting at most $k$ candidates other than $p$ ensure that $p$ is not a winner of the resulting $\mathscr{E}$ election?

\section{Control via PARTITION AND Run-OFF PARTITION OF CANDIDATES}

Bartholdi et al. (1992) gave two types of control of elections via partition of candidates. In both cases the candidate set $C$ is partitioned into two groups, $C_{1}$ and $C_{2}$ (i.e., $C_{1} \cup C_{2}=C$ and $C_{1} \cap C_{2}=\emptyset$ ), and the election is conducted in two stages. For control via run-off partition of candidates, the election's first stage is conducted separately on each group of candidates, $C_{1}$ and $C_{2}$, and the group winners that survive the tie-handling rule compete against each other in the second stage. In control via partition of candidates, the first-stage election is performed on the candidate set $C_{1}$ and those of that election's winners that survive the tie-handling rule compete against all candidates from $C_{2}$ in the second stage.

In the ties-promote (TP) model, all first-stage winners within a group are promoted to the final round. In the ties-eliminate (TE) model, a first-stage winner within a group is promoted to the final round if and only if he or she is the unique winner within that group.

Although these only loosely correspond to real-world settings, let us give a rough example regarding the case of run-off partition of candidates. Consider a department, with a powerful director, that is trying to decide among a collection of alternatives. It is certainly plausible that the director might announce that she had divided the candidates into two groups, that the entire department would vote separately among the candidates in each group, and that then only those candidates who moved forward from those votes (under whatever tie-handling rule was being used, if there were ties) would compete in the final election, in which the entire department would again vote. (How

6. A referee asked whether control by adding candidates, if redefined to require adding not at most a certain number of candidates but instead at least a certain number of candidates, can cover the forthcoming notion (which is the standard notion) of control by deleting (at most a certain number of) candidates. The answer is that that seems not to be the case. Consider an election with thirty candidates in which we ask whether a certain constructive control goal can be reached via deleting at most five candidates. Note that reframing this as a twenty-candidate election in which one tries to reach some goal by adding at least five candidates from a ten-candidate spoiler set doesn't make sense, as there is no one particular twenty-candidate election from which to start; there are far too many possibilities. The referee similarly asked about representing addition of candidates by a new notion of deleting candidates that put a lower bound on the number of deletions, but that attempt seems also to fail, in that case for the different reason that in the deletion case one might delete not just what originally were spoiler candidates but one might delete candidates from the core election of the addition case, and that is not allowed. 
convincingly the director could do this would of course depend on the director's power and how well the director could think up a justification for her partition of the candidates. Clearly some partitions may be easy to justify, e.g., "Let's regarding whom to hire in our academic computer science department first vote separately among the fresh-Ph.D. candidates and among the more senior hiring candidates," and some may be harder to justify except as executive fiat.)

Name: $\mathscr{E}$-CCRPC and $\mathscr{E}$-DCRPC (control via run-off partition of candidates).

Given: A set $C$ of candidates, a collection $V$ of voters represented via preference lists over $C$, and a distinguished candidate $p \in C$.

Question ( $\mathscr{E}$-CCRPC): Is there a partition of $C$ into $C_{1}$ and $C_{2}$ such that $p$ is a winner of the twostage election where the winners of subelection $\left(C_{1}, V\right)$ that survive the tie-handling rule compete against the winners of subelection $\left(C_{2}, V\right)$ that survive the tie-handling rule? Each subelection (in both stages) is conducted using election system $\mathscr{E}$.

Question ( $\mathscr{E}$-DCRPC): Is there a partition of $C$ into $C_{1}$ and $C_{2}$ such that $p$ is not a winner of the two-stage election where the winners of subelection $\left(C_{1}, V\right)$ that survive the tie-handling rule compete against the winners of subelection $\left(C_{2}, V\right)$ that survive the tie-handling rule? Each subelection (in both stages) is conducted using election system $\mathscr{E}$.

The above description defines four computational problems for a given election system $\mathscr{E}$ : $\mathscr{E}$-CCRPC-TE, $\mathscr{E}$-CCRPC-TP, $\mathscr{E}$-DCRPC-TE, and $\mathscr{E}$-DCRPC-TP. Note that it is in concept possible in the TE case for all candidates, due to ties, to be eliminated in the first round here, in which case the overall election would have no winner.

Name: $\mathscr{E}$-CCPC and $\mathscr{E}$-DCPC (control via partition of candidates).

Given: A set $C$ of candidates, a collection $V$ of voters represented via preference lists over $C$, and a distinguished candidate $p \in C$.

Question ( $\mathscr{E}$-CCPC): Is there a partition of $C$ into $C_{1}$ and $C_{2}$ such that $p$ is a winner of the two-stage election where the winners of subelection $\left(C_{1}, V\right)$ that survive the tie-handling rule compete against all candidates in $C_{2}$ ? Each subelection (in both stages) is conducted using election system $\mathscr{E}$.

Question ( $\mathscr{E}$-DCPC): Is there a partition of $C$ into $C_{1}$ and $C_{2}$ such that $p$ is not a winner of the two-stage election where the winners of subelection $\left(C_{1}, V\right)$ that survive the tie-handling rule compete against all candidates in $C_{2}$ ? Each subelection (in both stages) is conducted using election system $\mathscr{E}$.

This description defines four computational problems for a given election system $\mathscr{E}$ : $\mathscr{E}$-CCPC-TE, $\mathscr{E}$-CCPC-TP, $\mathscr{E}$-DCPC-TE, and $\mathscr{E}$-DCPC-TP.

\section{CONTROL Via AdDing Voters}

In the scenario of control via adding voters, the chair's goal is to either ensure that $p$ is a winner (in the constructive case) or ensure that $p$ is not a winner (in the destructive case) via causing up to $k$ 
additional voters to participate in the election. The chair can draw the voters to add to the election from a prespecified collection of voters (with given preferences).

This can very loosely model such real-world situations as get-out-the-vote efforts. For example, suppose a campaign has enough money and volunteers to drive up to one hundred from a set of a thousand car-less elderly people to the polling place, and has to decide which ones to choose.

Name: $\mathscr{E}-\mathrm{CCAV}$ and $\mathscr{E}$-DCAV (control via adding voters).

Given: A set $C$ of candidates, two disjoint collections of voters, $V$ and $W$, represented via preference lists over $C$, a distinguished candidate $p$, and a nonnegative integer $k$.

Question (E्E-CCAV): Is there a subset $Q,\|Q\| \leq k$, of voters in $W$ such that the voters in $V \cup Q$ jointly elect $p \in C$ as a winner according to system $\mathscr{E}$ ?

Question ( $\mathscr{E}$-DCAV): Is there a subset $Q,\|Q\| \leq k$, of voters in $W$ such that the voters in $V \cup Q$ do not elect $p$ as a winner according to system $\mathscr{E}$ ?

The reason we do not have an "unlimited" control notion here, or anywhere else except for $\mathrm{AC}_{\mathrm{u}}$, is that $\mathrm{AC}_{\mathrm{u}}$ is historically a special case. The seminal paper Bartholdi et al. 1992 defined all addition/deletion problems in (only) the limited version, in which there is number $k$ limiting the additions/deletions, except that their paper, describing this as a matter of individual taste, defined addition of candidates in (only) the unlimited version. We consider the limited versions of all the addition/deletion problems by far the more natural, and so we study those, as did Bartholdi, Tovey, and Trick in every case other than addition of candidates. However, to allow comparison with earlier papers, we keep as a defined control type the case of $\mathrm{AC}_{\mathrm{u}}$.

\section{CONTROl Via Deleting Voters}

In the control via deleting voters case the chair seeks to either ensure that $p$ is a winner (in the constructive case) or prevent $p$ from being a winner (in the destructive case) via blocking up to $k$ voters from participating in the election.

This very loosely models vote suppression. For example, consider the case where a given campaign can afford to send to the doors of at most $k$ voters a smooth-talking operative who will so demoralize them that they won't bother to vote.

Name: $\mathscr{E}$-CCDV and $\mathscr{E}$-DCDV (control via deleting voters).

Given: A set $C$ of candidates, a collection $V$ of voters represented via preference lists over $C$, a distinguished candidate $p \in C$, and a nonnegative integer $k$.

Question ( $\mathscr{E}-\mathrm{CCDV}):$ Is it possible to by deleting at most $k$ voters ensure that $p$ is a winner of the resulting $\mathscr{E}$ election?

Question ( $\mathscr{E}$-DCDV): Is it possible to by deleting at most $k$ voters ensure that $p$ is not a winner of the resulting $\mathscr{E}$ election? 


\section{CONTROL VIA PARTITION OF VOTERS}

In the case of control via partition of voters, the following two-stage election is performed. First, the voter set $V$ is partitioned into two subcommittees, $V_{1}$ and $V_{2}$. The winners of election $\left(C, V_{1}\right)$ that survive the tie-handling rule compete against the winners of $\left(C, V_{2}\right)$ that survive the tie-handling rule. Again, our tie-handling rules are TE and TP (ties-eliminate and ties-promote).

This control type is a bit harder than most others to imagine in the real world, but as a somewhat contrived example, consider the following case. In a given organization, the director splits her workers into two study groups (and let us say she can choose the partition as she likes, either because she is a powerful director, or because she is a good enough manager to make up a justification for any division) to each study a problem and to each propose what it thinks is the best alternative. And then the entire organization comes together to vote among those alternatives chosen in the first round (that survive the tie-handling rule in the case of ties).

Name: $\mathscr{E}$-CCPV and $\mathscr{E}$-DCPV (control via partition of voters).

Given: A set $C$ of candidates, a collection $V$ of voters represented via preference lists over $C$, and a distinguished candidate $p \in C$.

Question ( $\mathscr{E}$-CCPV): Is there a partition of $V$ into $V_{1}$ and $V_{2}$ such that $p$ is a winner of the twostage election where the winners of election $\left(C, V_{1}\right)$ that survive the tie-handling rule compete against the winners of $\left(C, V_{2}\right)$ that survive the tie-handling rule? Each subelection (in both stages) is conducted using election system $\mathscr{E}$.

Question ( $\mathscr{E}$-DCPV): Is there a partition of $V$ into $V_{1}$ and $V_{2}$ such that $p$ is not a winner of the twostage election where the winners of election $\left(C, V_{1}\right)$ that survive the tie-handling rule compete against the winners of $\left(C, V_{2}\right)$ that survive the tie-handling rule? Each subelection (in both stages) is conducted using election system $\mathscr{E}$.

\section{UNIQUE WINNERS AND IRRATIONALITY}

Our bribery and control problems were each defined above only for rational voters and in the nonunique-winner model, i.e., asking whether a given candidate can be made, or prevented from being, $a$ winner. Nonetheless, we have proven all our control results both for the case of nonunique winners and (to be able to fairly compare them with existing control results, which are in the uniquewinner model) unique winners (a candidate is a unique winner if he or she is a winner and is the only winner). Similarly, all our bribery results are proven both in the unique-winner model and (to be able to fairly compare them with existing bribery results in the literature) in the nonunique-winner model. In addition to the rational-voters case, we also study these problems for the case of voters who are allowed to be irrational. As mentioned earlier, in the case of irrational voters, voters are represented via preference tables rather than preference lists.

\subsection{Graphs}

An undirected graph $G$ is a pair $(V(G), E(G))$, where $V(G)$ is the set of vertices and $E(G)$ is the set of edges and each edge is an unordered pair of distinct vertices. ${ }^{7}$ A directed graph is defined

7. In this paper, the symbols $E$ and $V$ are generally reserved for elections and voters, except the just-introduced "overloading" of them to mean sets of edges and vertices in a given graph. The intended meaning of $E$ and $V$ will be clear from the context, even when our proofs involve multiple elections and graphs. 
analogously, except that the edges are represented as ordered pairs. For example, if $u$ and $v$ are distinct vertices in an undirected graph $G$ then $G$ either has an edge $e=\{u, v\}$ that connects $u$ and $v$ or it doesn't. On the other hand, if $G$ is a directed graph then $G$ either has an edge $e^{\prime}=(u, v)$ from $u$ to $v$, or an edge $e^{\prime \prime}=(v, u)$ from $v$ to $u$, or both $e^{\prime}$ and $e^{\prime \prime}$, or neither $e^{\prime}$ nor $e^{\prime \prime}$.

For a directed graph $G$, the indegree of a vertex $u \in V(G)$ is the number of $G$ 's edges that enter $u$ (i.e., the number of edges of the form $(v, u)$ in $E(G)$ ). Similarly, the outdegree of $u \in V(G)$ is the number of edges that leave $u$ (i.e., the number of edges of the form $(u, v)$ in $E(G)$ ).

\subsection{NP-Complete Problems and Reductions}

Without loss of generality, we assume that all problems that we consider are encoded in a natural, efficient way over the alphabet $\Sigma=\{0,1\}$. We use the standard notion of NP-completeness, defined via polynomial-time many-one reductions. We say that a computational problem A polynomial-time many-one reduces to a problem $B$ if there exists a polynomial-time computable function $f$ such that

$$
\left(\forall x \in \Sigma^{*}\right)[x \in A \Longleftrightarrow f(x) \in B]
$$

A problem is NP-hard if all members of NP polynomial-time many-one reduce to it. Thus, if an NP-hard problem $A$ polynomial-time many-one reduces to a problem $B$, then $B$ is NP-hard as well. A problem is NP-complete if it is NP-hard and is a member of NP. When clear from context we will use "reduce" and "reduction" as shorthands for "polynomial-time many-one reduce" and "polynomial-time many-one reduction."

Our NP-hardness results typically follow via a reduction from either the exact-cover-by-3-sets problem or from the vertex cover problem (see, e.g., Garey and Johnson, 1979). These are wellknown NP-complete problems, but we define them here for the sake of completeness.

Name: $\mathrm{X} 3 \mathrm{C}$ (exact cover by 3 -sets).

Given: A set $B=\left\{b_{1}, \ldots, b_{3 k}\right\}, k \geq 1$, and a family of sets $\mathscr{S}=\left\{S_{1}, \ldots, S_{n}\right\}$ such that for each $i$, $1 \leq i \leq n$, it holds that $S_{i} \subseteq B$ and $\left\|S_{i}\right\|=3$.

Question: Is there a set $A \subseteq\{1, \ldots, n\},\|A\|=k$, such that $\bigcup_{i \in A} S_{i}=B$ ?

The set $A$ about which we ask in the above problem is called an exact cover of $B$. It is a "cover" because every member of $B$ belongs to some $S_{i}$ such that $i \in A$, and it is "exact" because each member of $B$ belongs to exactly one $S_{i}$ such that $i \in A$.

Whenever we consider instances of the $\mathrm{X} 3 \mathrm{C}$ problem, we assume that they are well-formed, that is, we assume that they follow the syntactic requirements stated in the above "Given" field (e.g., the cardinality of the set $B$ is indeed a multiple of three). We apply this convention of considering only syntactically correct inputs to all other problems as well. Let $A$ be some computational problem and let $x$ be an instance of $A$. When we consider an algorithm for $A$, and input $x$ is malformed, then we can immediately reject. When we are building a reduction from $A$ to some problem $B$, then whenever we hit a malformed input $x$ we can output a fixed $y$ not in $B$. (In our reductions $B$ is never $\Sigma^{*}$, so this is always possible.)

Copeland ${ }^{\alpha}$ elections can often be considered in terms of appropriate graphs. This representation is particularly useful when we face control problems that modify the structure of the candidate set, since in this case operations on an election directly translate into suitable operations on the 
corresponding graph. For candidate control problems, we-instead of using reductions from $\mathrm{X} 3 \mathrm{C}-$ construct reductions from the vertex cover problem. A vertex cover of an undirected graph $G$ is a subset of $G$ 's vertices such that each edge of $G$ is adjacent to at least one vertex from that subset.

Name: VertexCover.

Given: An undirected graph $G$ and a nonnegative integer $k$.

Question: Is there a set $W$ such that $W \subseteq V(G),\|W\| \leq k$, and for every edge $e \in E(G)$ it holds that $e \cap W \neq \emptyset$ ?

\subsection{Resistance and Vulnerability}

Not all election systems can be affected by each control type; if not, the system is said to be immune to this type of control. For example, if a candidate $c$ is not a Condorcet winner then it is impossible to make him or her a Condorcet winner by adding candidates (see Bartholdi et al., 1992, and Hemaspaandra et al., 2007a, for more such immunity results). However, for Copeland ${ }^{\alpha}$ elections it is easy to see that for each type of control defined in Section 2.2 there is a scenario in which the outcome of the election can indeed be changed via conducting the corresponding control action. If an election system is not immune to some type of control (as witnessed by such a scenario), the election system is said to be susceptible to that control type.

Proposition 2.4 For each rational number $\alpha, 0 \leq \alpha \leq 1$, Copeland ${ }^{\alpha}$ is susceptible to each type of control defined in Section 2.2.

We say that an election system $\left(\right.$ Copeland $^{\alpha}$ or Copeland $\mathrm{Irrational}^{\alpha}$, in our case) is resistant to a particular attack (be it a type of control or of bribery) if the appropriate computational problem is NP-hard and susceptibility holds. ${ }^{8}$ On the other hand, if the computational problem is in $\mathrm{P}$ and susceptibility holds, then we say the system is vulnerable to this attack. Because of how our bribery and control problems are defined, the vulnerability definition merely requires that there exist a polynomial-time algorithm that determines whether a successful bribe or control action exists on a given input. However, in every single one of our vulnerability proofs we will provide something far stronger. We will provide a polynomial-time algorithm that actually finds a successful bribe or control action on each input for which a successful bribe or control action exists, and on each input where no successful bribe or control action exists will announce that fact.

The notions of resistance and vulnerability (and of immunity and susceptibility) for control problems in election systems were introduced by Bartholdi et al. (1992), and we here follow the definition alteration of Hemaspaandra et al. (2007b) of resistance from "NP-complete" to "NPhard," as that change is compelling (because under the old definition, NP-completeness, things could

8. It is true that for some unnatural election systems immunity to bribery holds, e.g., the election system "Every candidate is a winner" is immune to all types of bribery. However, our Copeland ${ }^{\alpha}$-type systems are all susceptible to all the bribery types we look at in this paper, so we won't further explicitly discuss or state susceptibility for the bribery cases.

A referee asked whether the definition of resistance could be equivalently stated as simply requiring the appropriate computational problem to be NP-hard. That seems not to yield the same notion, both because $\mathrm{P} \neq \mathrm{NP}$ is not yet a known result, and so one doesn't know that NP-hard problems cannot possibly be in P, and more subtly because susceptibility is defined in terms of changing outcomes while the corresponding control problem's NP-hardness (which in part determines its resistance) is related to what the outcome is (regardless of what it started as). 
actually become nonresistant by being too hard, which is not natural). However, for all resistance claims in this paper NP-membership is clear, and so NP-completeness in fact does hold.

\section{Bribery}

In this section we present our results on the complexity of bribery for the Copeland ${ }^{\alpha}$ election systems, where $\alpha$ is a rational number with $0 \leq \alpha \leq 1$. Our main result, which will be presented in Section 3.1, is that each such system is resistant to bribery, regardless of voters' rationality and of our mode of operation (constructive versus destructive). In Section 3.2, we will provide vulnerability results for Llull and Copeland ${ }^{0}$ with respect to "microbribery."

\subsection{Resistance to Bribery}

Theorem 3.1 For each rational $\alpha, 0 \leq \alpha \leq 1$, Copeland ${ }^{\alpha}$ and Copeland $_{\text {Irrational }}^{\alpha}$ are resistant to both constructive and destructive bribery, in both the nonunique-winner model and the unique-winner model.

We prove Theorem 3.1 via Theorems 3.2, 3.4, and 3.5 below. Our proofs employ an approach that we call the UV technique. For the constructive cases, this technique proceeds by constructing bribery instances where the only briberies that could possibly ensure that our favorite candidate $p$ is a winner involve only voters who rank a group of special candidates (often the group will contain exactly two candidates, $u$ and $v$ ) above $p$. The remaining voters, the bystanders so to speak, can be used to create appropriate padding and structure within the election. The destructive cases follow via a cute observation regarding the dynamics of our constructive cases.

The remainder of this section is devoted to proving Theorem 3.1. We start with the case of rational voters in Theorems 3.2 and 3.4 below and then argue that the analogous results for the case of irrational voters follow via, essentially, the same proof.

Theorem 3.2 For each rational number $\alpha, 0 \leq \alpha \leq 1$, Copeland ${ }^{\alpha}$ is resistant to constructive bribery in the unique-winner model and to destructive bribery in the nonunique-winner model.

Proof. Fix an arbitrary rational number $\alpha$ with $0 \leq \alpha \leq 1$. Our proof provides reductions from the $\mathrm{X} 3 \mathrm{C}$ problem to, respectively, the unique-winner variant of constructive bribery and to the nonunique-winner variant of destructive bribery. Our reductions will differ regarding only the specification of the goal (i.e., regarding which candidate we attempt to make a unique winner or which candidate we prevent from being a winner) and thus we describe them jointly as, essentially, a single reduction.

Our reduction will produce an instance of an appropriate bribery problem with an odd number of voters, and so we will never have ties in head-to-head contests. Thus our proof works regardless of which rational number $\alpha$ with $0 \leq \alpha \leq 1$ is chosen.

Let $(B, \mathscr{S})$ be an instance of X3C, where $B=\left\{b_{1}, b_{2}, \ldots, b_{3 k}\right\}, \mathscr{S}$ is a collection $\left\{S_{1}, S_{2}, \ldots, S_{n}\right\}$ of three-element subsets of $B$ with $\bigcup_{j=1}^{n} S_{j}=B$, and $k \geq 1$. If our input does not meet these conditions then we output a fixed instance of our bribery problem having a negative answer.

Construct a Copeland ${ }^{\alpha}$ election $E=(C, V)$ as follows. The candidate set $C$ is $\{u, v, p\} \cup B$, where none of $u, v$, and $p$ is in $B$. The voter set $V$ contains $2 n+4 k+1$ voters of the following types. 
1. For each $S_{i}$, we introduce one voter of type (i) and one voter of type (ii):

$$
\begin{aligned}
& \text { (i) } u>v>S_{i}>p>B-S_{i} \text {, } \\
& \text { (ii) } \overleftarrow{B-S_{i}}>p>u>v>\overleftarrow{S_{i}}
\end{aligned}
$$

2. We introduce $k$ voters for each of the types (iii)-1, (iii)-2, (iv)-1, and (iv)-2:

$$
\begin{array}{ll}
\text { (iii)-1 } & u>v>p>B \\
\text { (iii)-2 } & v>u>p>B \\
\text { (iv)-1 } & u>\overleftarrow{B}>p>v \\
\text { (iv)-2 } & v>\overleftarrow{B}>p>u
\end{array}
$$

3. We introduce a single type (v) voter:

$$
\text { (v) } B>p>u>v \text {. }
$$

We have the following relative vote-scores:

1. $\operatorname{vs}_{E}(u, v)=2 n+1 \geq 2 k+1$, where the inequality follows from our assumption $\bigcup_{j=1}^{n} S_{j}=B$ (which implies $n \geq\|B\| / 3=k$ ),

2. $\operatorname{vs}_{E}(u, p)=\operatorname{vs}_{E}(v, p)=2 k-1$,

3. for each $i \in\{1,2, \ldots, 3 k\}, \operatorname{vs}_{E}\left(u, b_{i}\right)=\operatorname{vs}_{E}\left(v, b_{i}\right) \geq 2 k+1$,

4. for each $i \in\{1,2, \ldots, 3 k\}, \operatorname{vs}_{E}\left(b_{i}, p\right)=1$, and

5. for each $i, j \in\{1,2, \ldots, 3 k\}$ with $i \neq j,\left|\operatorname{vs}_{E}\left(b_{i}, b_{j}\right)\right|=1$.

For example, to see that $\operatorname{vs}_{E}\left(u, b_{i}\right) \geq 2 k+1$ for each $i \in\{1,2, \ldots, 3 k\}$, note that each $b_{i}$ is in at least one $S_{j}$ because of $\bigcup_{j=1}^{n} S_{j}=B$, so the voters of types (i) and (ii) give $u$ an advantage of at least two votes over $b_{i}$. Furthermore, the voters of types (iii)-1, (iii)-2, (iv)-1, and (iv)-2 give $u$ an advantage of $2 k$ additional votes over each $b_{i}$, and the single type (v) voter gives each $b_{i}$ a one-vote advantage over $u$. Summing up, we obtain $\operatorname{vs}_{E}\left(u, b_{i}\right) \geq 2+2 k-1=2 k+1$. The other relative vote-scores are similarly easy to verify.

These relative vote-scores yield the following Copeland ${ }^{\alpha}$ scores or upper bounds on such scores:

1. $\operatorname{score}_{E}^{\alpha}(u)=3 k+2$,

2. $\operatorname{score}_{E}^{\alpha}(v)=3 k+1$,

3. for each $i \in\{1,2, \ldots, 3 k\}$, $\operatorname{score}_{E}^{\alpha}\left(b_{i}\right) \leq 3 k$, and

4. $\operatorname{score}_{E}^{\alpha}(p)=0$.

To prove our theorem, we need the following claim.

Claim 3.3 The following three statements are equivalent:

1. $(B, \mathscr{S}) \in X 3 C$. 


\section{Candidate u can be prevented from winning via bribing at most $k$ voters of $E$.}

3. Candidate $p$ can be made a unique winner via bribing at most $k$ voters of $E$.

Proof of Claim 3.3. (1) implies (2): It is easy to see that if $(B, \mathscr{S}) \in \mathrm{X} 3 \mathrm{C}$ then there is a bribe involving $k$ or fewer voters that prevents $u$ from being a winner: It is enough to bribe those type (i) voters that correspond to a cover of size $k$ to report $p$ as their top choice (while not changing anything else in their preference lists): $p>u>v>S_{i}>B-S_{i}$. Call the resulting election $E^{\prime}$. In $E^{\prime}$ the following relative vote-scores change: $\mathrm{vs}_{E^{\prime}}(p, u)=\mathrm{vs}_{E^{\prime}}(p, v)=n+k-(n-k)-2 k+1=1$ and $\operatorname{vs}_{E^{\prime}}\left(p, b_{i}\right) \geq 1$ for each $i \in\{1,2, \ldots, 3 k\}$, while all other relative vote-scores remain unchanged. Thus $\operatorname{score}_{E^{\prime}}^{\alpha}(p)=3 k+2, \operatorname{score}_{E^{\prime}}^{\alpha}(u)=3 k+1, \operatorname{score}_{E^{\prime}}^{\alpha}(v)=3 k$, and $\operatorname{score}_{E^{\prime}}^{\alpha}\left(b_{i}\right)<3 k$ for each $i \in\{1,2, \ldots, 3 k\}$, so $p$ defeats all other candidates and is the unique winner. In particular, this bribe (of at most $k$ voters in $E$ ) ensures that $u$ is not a winner.

(2) implies (3): Suppose that there is a bribe involving $k$ or fewer voters that prevents $u$ from being a winner. Note that $u$ defeats everyone except $p$ by more than $2 k$ votes in $E$. This means that via bribery of at most $k$ voters $u$ 's score can decrease by at most one. Thus, to prevent $u$ from being a winner via such a bribery, we need to ensure that $u$ receives a Copeland ${ }^{\alpha}$ score of $3 k+1$ and some candidate other than $u$ gets a Copeland ${ }^{\alpha}$ score of $3 k+2$, that is, that candidate defeats everyone. Neither $v$ nor any of the $b_{i}$ 's can possibly obtain a Copeland ${ }^{\alpha}$ score of $3 k+2$ via such a bribery, since bribery of at most $k$ voters can affect only head-to-head contests where the relative vote-scores of the participants are at most $2 k$. Thus, via such a bribery, $u$ can be prevented from winning only if $p$ can be made a (in fact, the unique) winner of our election.

(3) implies (1): Let $W$ be a set of at most $k$ voters whose bribery ensures that $p$ is a unique winner of our election. Thus we know that $\|W\|=k$ and that $W$ contains only voters who rank both $u$ and $v$ above $p$ (as otherwise $p$ would not defeat both $u$ and $v$ ), which is the case only for voters of types (i), (iii)-1, and (iii)-2. Furthermore, a bribery that makes $p$ the unique winner has to ensure that $p$ defeats all members of $B$; note that the type (iii)-1 and (iii)-2 voters in $E$ already rank $p$ above all of $B$. Thus, via a simple counting argument, $W$ must contain exactly $k$ type (i) voters that correspond to a size- $k$ cover of $B$.

ㄷaim 3.3

Since our reduction is computable in polynomial time, Claim 3.3 completes the proof of Theorem 3.2.

Theorem 3.4 For each rational $\alpha, 0 \leq \alpha \leq 1$, Copeland ${ }^{\alpha}$ is resistant to constructive bribery in the nonunique-winner model and to destructive bribery in the unique-winner model.

The proof of Theorem 3.4, which follows the same general structure as the proof of Theorem $3.2,{ }^{9}$ for reasons of space and nonrepetitiveness is not included here but can be found in the full TR version (Faliszewski et al., 2008b).

9. Since the proof of Theorem 3.4 is slightly more involved, let us briefly mention its key differences from the proof of Theorem 3.2. Starting from an $\mathrm{X} 3 \mathrm{C}$ instance $(B, \mathscr{S})$ with $\|B\|=3 k$, we in this case construct an election $E=(C, V)$ with two more candidates (i.e., $C=\{p, s, t, u, v\} \cup B$ ) and with $V$ having, in addition to the voter types similar to those in the proof of Theorem 3.2, $20 \mathrm{k}$ "normalizing" voters of eight subtypes. The unique winner of $E$ is $s$, and the only candidate who is able to prevent $s$ from being the unique winner via at most $k$ voters being bribed is $p$. The construction ensures that $(B, \mathscr{S}) \in \mathrm{X} 3 \mathrm{C}$ exactly if at most $k$ voters can be bribed such that $p$ and $s$ tie for winner, which simultaneously handles the nonunique-winner constructive case and the unique-winner destructive case. 
The proofs of the above theorems have an interesting feature. When we discuss bribery, we never rely on the fact that the voters are rational. Thus we can allow the voters to be irrational and form Copeland ${ }_{\text {Irrational }}^{\alpha}$-bribery and Copeland $\mathrm{Irrational}^{\alpha}$-destructive-bribery instances simply by deriving the voters' preference tables from the voters' preference lists given in the above proofs. It is easy to see that the proofs remain valid after this change; in the proofs we assume that each bribed voter, after the bribery, prefers $p$ to all other candidates, but we do not make any further assumptions (and, in particular, we do not use linearity of the preferences). Thus we have the following corollary to the proofs of Theorems 3.2 and 3.4.

Theorem 3.5 For each rational number $\alpha, 0 \leq \alpha \leq 1$, Copeland $\mathrm{d}_{\text {Irrational }}^{\alpha}$ is resistant to both constructive bribery and destructive bribery, in both the nonunique-winner model and the uniquewinner model.

Theorems 3.2, 3.4, and Theorem 3.5 together constitute a proof of Theorem 3.1 and establish that for each rational $\alpha, 0 \leq \alpha \leq 1$, Copeland ${ }^{\alpha}$ and Copeland Irrational $^{\alpha}$ possess broad-essentially perfect - resistance to bribery regardless of whether we are interested in constructive or destructive results. However, the next section shows that this perfect picture is, in fact, only near-perfect when we consider microbribes, which don't allow changing the complete preferences of voters at once but rather change the results of head-to-head contests between candidates in the voters' preferences. We will show that there is an efficient way of finding optimal microbriberies for the case of irrational voters in Copeland ${ }^{\alpha}$ elections.

\subsection{Vulnerability to Microbribery for Irrational Voters}

In this section we explore the problems related to microbribery of irrational voters. In standard bribery problems, which were considered in Section 3.1, we ask whether it is possible to ensure that a designated candidate $p$ is a winner (or, in the destructive case, to ensure that $p$ is not a winner) via modifying the preference tables of at most $k$ voters. That is, we can at unit cost completely redefine the preference table of each voter bribed. So in this model, we pay for a service (namely, the modification of the reported preference table) and we pay for it in bulk (when we buy a voter, we have secured his or her total obedience). However, sometimes it may be far more reasonable to adopt a more local approach in which we have to pay separately for each preference-table entry flip-to pay more the more we alter a vote.

Throughout the remainder of this section we will use the term microbribe to refer to flipping an entry in a preference table, and we will use the term microbribery to refer to bribing possibly irrational voters via microbribes. Recall that by "irrational voters" we simply mean that they are allowed to have, but not that they must have, irrational preferences.

For the study of microbribery, we consider irrational voters to clearly be the natural model to study. After all, one is changing (and measuring the overall change in terms of the number of changes in) pairwise preferences, and such changes can easily move one from a rational preference to an irrational preference. (We mention in passing that one could define versions of this problem for the case of rational voters in various ways, e.g., allowing only changes that stay on rational profiles. But that seems a far less natural model to use for the microbribery problem.)

For each rational $\alpha, 0 \leq \alpha \leq 1$, we define the following two problems.

Name: Copeland ${ }_{\text {Irrational }}^{\alpha}$-microbribery and Copeland $\mathrm{Irrational}^{\alpha}{ }^{- \text {destructive-microbribery. }}$ 
Given: A set $C$ of candidates, a collection $V$ of voters specified via their preference tables over $C$, a distinguished candidate $p \in C$, and a nonnegative integer $k$.

Question (constructive): Is it possible, by flipping at most $k$ entries in the preference tables of voters in $V$, to ensure that $p$ is a winner of the resulting election?

Question (destructive): Is it possible, by flipping at most $k$ entries in the preference tables of voters in $V$, to guarantee that $p$ is not a winner of the resulting election?

We can flip multiple entries in the preference table of the same voter, but we have to pay separately for each flip. The microbribery problems for Copeland ${ }_{\text {Irrational }}^{\alpha}$ are very similar in flavor to the so-called bribery' problems for approval voting that were studied by Faliszewski et al. (2006a), where unit cost for flipping approvals or disapprovals of voters are paid. However, the proofs for Copeland $_{\text {Irrational }}^{\alpha}$ seem to be much more involved than their counterparts for approval voting. The reason is that Copeland ${ }_{\text {Irrational }}^{\alpha}$ elections allow for very subtle and complicated interactions between the candidates' scores.

Before we proceed with our results, let us define some notation that will be useful throughout this section. Let $E$ be an election with candidate set $C=\left\{c_{1}, c_{2}, \ldots, c_{m}\right\}$ and voter collection $V=$ $\left\{v_{1}, v_{2}, \ldots, v_{n}\right\}$. We define two functions, wincost $_{E}$ and tiecost $_{E}$, that describe the costs of ensuring a victory or a tie of a given candidate in a particular head-to-head contest.

Definition 3.6 Let $E=(C, V)$ be an election and let $c_{i}$ and $c_{j}$ be two distinct candidates in $C$.

1. By wincost ${ }_{E}\left(c_{i}, c_{j}\right)$ we mean the minimum number of microbribes that ensure that $c_{i}$ defeats $c_{j}$ in their head-to-head contest. If $c_{i}$ already wins this contest then wincost $E_{E}\left(c_{i}, c_{j}\right)=0$.

2. By tiecost ${ }_{E}\left(c_{i}, c_{j}\right)$ we mean the minimum number of microbribes that ensure that $c_{i}$ ties with $c_{j}$ in their head-to-head contest, or $\infty$ if $E$ has an odd number of voters and thus ties are impossible.

Our first result regarding microbribery is that destructive microbribery is easy for Copeland $\mathrm{Irrational}^{\alpha}$. Since this is the paper's first vulnerability proof, we take this opportunity to remind the reader (recall Section 2.5) that although the definition of vulnerability requires only that there be a polynomial-time algorithm to determine whether a successful action (in the present case, a destructive microbribery) exists, we will in each vulnerability proof provide something far stronger, namely a polynomial-time algorithm that both determines whether a successful action exists and that, when so, finds a successful action (e.g., for our flow algorithms later on, the successful action will be implicit in the flow computed).

Theorem 3.7 For each rational $\alpha, 0 \leq \alpha \leq 1$, Copeland ${ }_{\text {Irrational }}^{\alpha}$ is vulnerable to destructive microbribery in both the nonunique-winner model and the unique-winner model.

Proof. Fix an arbitrary rational number $\alpha$ with $0 \leq \alpha \leq 1$. We give an algorithm for Copeland ${ }_{\text {Irrational }}^{\alpha}$, for destructive microbribery in the nonunique-winner model. (We omit the analogous algorithm for the unique-winner case.)

Let $E=(C, V)$ be the input election where $C=\left\{p, c_{1}, c_{2}, \ldots, c_{m}\right\}$ and $V=\left\{v_{1}, v_{2}, \ldots, v_{n}\right\}$, and let $k$ be the number of microbribes that we are allowed to make. We define the predicate 
$M\left(E, p, c_{i}, k\right)$ to be true if and only if there is a microbribery of cost at most $k$ that ensures that $c_{i}$ 's score is higher than that of $p$. Our algorithm computes $M\left(E, p, c_{i}, k\right)$ for each $c_{i} \in C$ and accepts if and only if it is true for at least one of them. We now describe how to compute $M\left(E, p, c_{i}, k\right){ }^{10}$

By applying appropriate minimum-cost microbriberies to $E$, we obtain elections $E_{1}, E_{2}$, and $E_{3}$ that are identical to $E$ except that

1. in $E_{1}, p$ defeats $c_{i}$ in their head-to-head contest,

2. in $E_{2}, p$ loses to $c_{i}$ in their head-to-head contest, and

3. in $E_{3}, p$ ties $c_{i}$ in their head-to-head contest (we disregard $E_{3}$ if the number of voters is odd and thus ties are impossible).

Let $k_{1}, k_{2}$, and $k_{3}$ be the minimum costs of microbriberies that transform $E$ to $E_{1}, E$ to $E_{2}$, and $E$ to $E_{3}$, respectively. Such microbriberies involve only the head-to-head contest between $p$ and $c_{i}$. We define the predicate $M^{\prime}\left(E^{\prime}, p, c_{i}, k^{\prime}\right)$, where $E^{\prime} \in\left\{E_{1}, E_{2}, E_{3}\right\}$ and where $k^{\prime}$ is an integer, to be true if and only if there is a microbribery of cost at most $k^{\prime}$ that does not involve the head-to-head contest between $p$ and $c_{i}$ but that ensures that $c_{i}$ 's Copeland ${ }_{\text {Irrational }}^{\alpha}$ score is higher than $p$ 's. It is easy to see that

$$
M\left(E, p, c_{i}, k\right) \Longleftrightarrow\left(M^{\prime}\left(E_{1}, p, c_{i}, k-k_{1}\right) \vee M^{\prime}\left(E_{2}, p, c_{i}, k-k_{2}\right) \vee M^{\prime}\left(E_{3}, p, c_{i}, k-k_{3}\right)\right) .
$$

Thus it is enough to focus on the problem of computing $M^{\prime}\left(E^{\prime}, p, c_{i}, k^{\prime}\right)$.

Let $\left(E^{\prime}, k^{\prime}\right)$ be one of $\left(E_{1}, k-k_{1}\right),\left(E_{2}, k-k_{2}\right)$, and $\left(E_{3}, k-k_{3}\right)$. Define promote $_{E^{\prime}}\left(c_{i}, w^{\prime}, w^{\prime \prime}, t\right)$, where $c_{i} \in C$ is a candidate and $w^{\prime}, w^{\prime \prime}$, and $t$ are nonnegative integers, to be the minimum cost of a microbribery that, when applied to $E^{\prime}$, increases $c_{i}$ 's Copeland ${ }_{\text {Irrational }}^{\alpha}$ score by $w^{\prime}+(1-\alpha) w^{\prime \prime}+\alpha t$ via ensuring that

1. $c_{i}$ wins an additional $w^{\prime}$ head-to-head contests against candidates in $C-\{p\}$ that used to defeat $c_{i}$ originally,

2. $c_{i}$ wins an additional $w^{\prime \prime}$ head-to-head contests against candidates in $C-\{p\}$ with whom $c_{i}$ used to tie originally, and

3. $c_{i}$ ties an additional $t$ head-to-head contests with candidates in $C-\{p\}$ that used to defeat $c_{i}$ originally.

If such a microbribery does not exist then we set promote $_{E^{\prime}}\left(c_{i}, w^{\prime}, w^{\prime \prime}, t\right)$ to be $\infty$. It is an easy

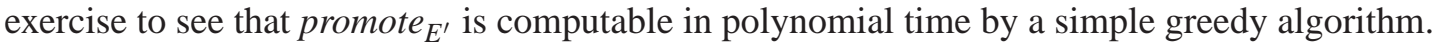

We define demote $_{E^{\prime}}\left(c_{i}, \ell^{\prime}, \ell^{\prime \prime}, t\right)$ to be the minimum cost of a microbribery that, when applied to election $E^{\prime}$, decreases $p$ 's score by $\ell^{\prime}+\alpha \ell^{\prime \prime}+(1-\alpha) t$ via ensuring that

1. $p$ loses an additional $\ell^{\prime}$ head-to-head contests to candidates in $C-\left\{c_{i}\right\}$ whom $p$ used to defeat originally,

2. $p$ loses an additional $\ell^{\prime \prime}$ head-to-head contests to candidates in $C-\left\{c_{i}\right\}$ with whom $p$ used to tie originally, and

10. We stress that we have optimized our algorithm for simplicity rather than for performance. 
3. $p$ ties an additional $t$ head-to-head contests with candidates in $C-\left\{c_{i}\right\}$ whom $p$ used to defeat originally.

If such a microbribery does not exist then we set demote $_{E^{\prime}}\left(c_{i}, \ell^{\prime}, \ell^{\prime \prime}, t\right)$ to be $\infty$. Note that demote $_{E^{\prime}}$ can be computed in polynomial time using an algorithm similar to that for promote $E_{E^{\prime}}$.

Naturally, the microbriberies used implicitly within promote $_{E^{\prime}}\left(c_{i}, w^{\prime}, w^{\prime \prime}, t^{\prime}\right)$, within demote $_{E^{\prime}}\left(c_{i}, \ell^{\prime}, \ell^{\prime \prime}, t^{\prime \prime}\right)$, and within transforming $E$ to $E^{\prime}$ are "disjoint," i.e., they never involve the same pair of candidates. Thus $M^{\prime}\left(E^{\prime}, p, c_{i}, k^{\prime}\right)$ is true if and only if there exist integers $w^{\prime}, w^{\prime \prime}, \ell^{\prime}, \ell^{\prime \prime}, t^{\prime}, t^{\prime \prime} \in\{0,1, \ldots, m\}$ such that

$$
\operatorname{score}_{E^{\prime}}^{\alpha}\left(c_{i}\right)+\left(w^{\prime}+\ell^{\prime}+(1-\alpha)\left(t^{\prime \prime}+w^{\prime \prime}\right)+\alpha\left(t^{\prime}+\ell^{\prime \prime}\right)\right)-\operatorname{score}_{E^{\prime}}^{\alpha}(p)>0
$$

and

$$
\text { promote }_{E^{\prime}}\left(c_{i}, w^{\prime}, w^{\prime \prime}, t^{\prime}\right)+\text { demote }_{E^{\prime}}\left(c_{i}, \ell^{\prime}, \ell^{\prime \prime}, t^{\prime \prime}\right) \leq k .
$$

There are only polynomially many combinations of such $w^{\prime}, w^{\prime \prime}, \ell^{\prime}, \ell^{\prime \prime}, t^{\prime}$, and $t^{\prime \prime}$, and we can try them all. Thus we have given a polynomial-time algorithm for $M^{\prime}\left(E^{\prime}, p, c_{i}, k^{\prime}\right)$. Via the observations given at the beginning of our proof this implies that $M\left(E, p, c_{i}, k\right)$ is computable in polynomial time and the proof is complete.

The above destructive-case algorithm and approach is fairly straightforward; in the destructive case we do not need to worry about any side effects of promoting $c$ and demoting $p$. The constructive case is more complicated, but we still are able to obtain polynomial-time algorithms via a fairly involved use of flow networks to model how particular points shift between candidates. In the remainder of this section we restrict ourselves to the values $\alpha \in\{0,1\}$ or settings where the number of voters is odd and so ties never happen. We remind the reader that Copeland ${ }^{1}$ and Copeland ${ }_{\text {Irrational }}^{1}$, respectively, refer to Llull voting.

A flow network is a network of nodes with directed edges through which we want to transport some amount of flow from the source to the sink (these are two designated nodes). Each edge $e$ can carry up to $c(e)$ units of flow, and transporting each unit of flow through $e$ costs $a(e)$. In the min-cost-flow problem we have a target flow value $F$, and the goal is to find a way of transporting $F$ units of flow from the source to the sink, while minimizing the cost. (If there is no way of achieving target flow $F$, the cost in effect is infinite.)

We now define the notions related to flow networks more formally. Let $\mathbb{N}=\{0,1,2, \ldots\}$ and $\mathbb{Z}=\{\ldots,-2,-1,0,1,2, \ldots\}$.

Definition 3.8 1. A flow network is a quintuple $(K, s, t, c, a)$, where $K$ is a set of nodes that includes the source $s$ and the sink $t, c: K^{2} \rightarrow \mathbb{N}$ is the capacity function, and $a: K^{2} \rightarrow \mathbb{N}$ is the cost function. We assume that $c(u, u)=a(u, u)=0$ for each node $u \in K$, and that at most one of $c(u, v)$ and $c(v, u)$ is nonzero for each pair of distinct nodes $u, v \in K$. We also assume that if $c(u, v)=0$ then $a(u, v)=0$ as well.

2. Given a flow network $(K, s, t, c, a)$, a flow is a function $f: K^{2} \rightarrow \mathbb{Z}$ that satisfies the following conditions:

(a) For each $u, v \in K$, we have $f(u, v) \leq c(u, v)$, i.e., capacities limit the flow. 
(b) For each $u, v \in K$, we have $f(u, v)=-f(v, u) .{ }^{11}$

(c) For each $u \in K-\{s, t\}$, we have $\sum_{v \in K} f(u, v)=0$, i.e., the flow is conserved in all nodes except the source and the sink.

3. The value of flow $f$ is:

$$
\text { flowvalue }(f)=\sum_{v \in K} f(s, v) \text {. }
$$

The particular flow network we have in mind will always be clear from the context and so we will not indicate it explicitly (we will not write it explicitly as a subscript to the function flowvalue).

4. The cost of flow $f$ is defined as:

$$
\text { flowcost }(f)=\sum_{u, v \in K} a(u, v) f(u, v) .
$$

That is, we pay the price $a(u, v)$ for each unit of flow that passes from node u to node $v$.

Given a flow network $(K, s, t, c, a)$ we will often use the term edges to refer to pairs of distinct nodes $(u, v) \in K^{2}$ for which $c(u, v)>0$.

Below we define the min-cost-flow problem, which is well known from the literature. The definition we employ here is not the most general one but will suffice for our needs. (Readers seeking a broader discussion of the problem may wish to see, for example, the monograph Ahuja, Magnanti, and Orlin, 1993.)

Definition 3.9 We define the min-cost-flow problem as follows: Given a flow network $(K, s, t, c, a)$ and a target flow value $F$, find a flow $f$ that has value $F$ (if one exists) and has minimum cost among all such flows, or otherwise indicate that no such flow $f$ exists.

The min-cost-flow problem has a polynomial-time algorithm. ${ }^{12}$ There is a large body of work devoted to flow problems and we will not even attempt to provide a complete list of references here. Instead, we again point the reader to the excellent monograph Ahuja et al. 1993, which provides descriptions of polynomial-time algorithms, theoretical analysis, and numerous references to previous work on flow-related problems. We also mention that the issue of flows is so prevalent in the study of algorithms that the textbook Cormen, Leiserson, Rivest, and Stein 2001, on its page 787, contains an exposition of the min-cost-flow problem.

Coming back to the study of constructive microbribery for Llull and Copeland ${ }^{0}$, with irrational voters allowed, we now present the following result.

Theorem 3.10 For $\alpha \in\{0,1\}$, Copeland ${ }_{\text {Irrational }}^{\alpha}$ is vulnerable to constructive microbribery, in both the nonunique-winner model and the unique-winner model.

11. Note that each flow is fully defined via its nonnegative values. Whenever we speak of a flow (e.g., when defining some particular flows) we will just speak of its nonnegative part.

12. The min-cost-flow problem is often defined in terms of capacity and cost functions that are not necessarily limited to nonnegative integer values and so the corresponding flows are not restricted to integer values either. However, crucially for us, it is known that if the capacity and cost functions have integral values (as we have assumed) then there exist optimal solutions to the min-cost-flow problem that use only integer-valued flows and that can be found in polynomial time. 


\begin{tabular}{|l|l|}
\hline \multicolumn{1}{|c|}{ Edge } & \multicolumn{1}{c|}{ Parameters } \\
\hline \hline$e=\left(s, c_{i}\right)$, & $c(e)=$ score $_{E}^{\alpha}\left(c_{i}\right)$ \\
where $c_{i} \in C$ & $a(e)=0$ \\
\hline$e=\left(c_{i}, c_{j}\right)$, & $c(e)=1$ \\
where $c_{i}, c_{j} \in C$ and $\operatorname{vs}_{E}\left(c_{i}, c_{j}\right)>0$ & $a(e)=$ wincost $_{E}\left(c_{j}, c_{i}\right)$ \\
\hline \multirow{2}{*}{$e=\left(c_{0}, t\right)$} & $c(e)=T$ \\
& $a(e)=0$ \\
\hline$e=\left(c_{i}, t\right)$, & $c(e)=T$ \\
where $i>0$ and $c_{i} \in C$ & $a(e)=B$ \\
\hline \multirow{2}{*}{ every other edge $e$} & $c(e)=0$ \\
& $a(e)=0$ \\
\hline
\end{tabular}

Figure 1: Edge capacities and costs for min-cost-flow instance $I(T)$, built from election $E$.

We prove Theorem 3.10 via Lemmas 3.11 through 3.16 below, which cover three cases: (a) an odd number of voters, where all Copeland ${ }_{\text {Irrational }}^{\alpha}$ elections with $0 \leq \alpha \leq 1$ are identical due to the lack of ties, (b) Copeland $\mathrm{Irrational}^{1}$ with an even number of voters, and (c) Copeland $\mathrm{d}_{\text {Irrational }}^{0}$ with an even number of voters. These lemmas only discuss the nonunique-winner model but in each case it is easy to see how to change the algorithms and proofs to make them work for the unique-winner model.

Lemma 3.11 For each rational $\alpha$ with $0 \leq \alpha \leq 1$, there is a polynomial-time algorithm that solves the constructive microbribery problem for Copeland ${ }_{\text {Irrational }}^{\alpha}$ elections with an odd number of voters (in the nonunique-winner model).

Proof. Our input is a nonnegative integer $k$ (the budget) and an election $E=(C, V)$, where the candidate set $C$ is $\left\{c_{0}, c_{1}, \ldots, c_{m}\right\}$, the number of voters is odd, and $p=c_{0}$ is the candidate whose victory we want to ensure via at most $k$ microbribes. Note that we interchangeably use $p$ and $c_{0}$ to refer to the same candidate, since it is sometimes convenient to be able to speak of $p$ and all other candidates uniformly. As the number of voters is odd, ties never occur. Thus any candidate $c_{i}$ has the same Copeland ${ }_{\text {Irrational }}^{\alpha}$ score for each rational value of $\alpha, 0 \leq \alpha \leq 1$. Fix an arbitrary such $\alpha$.

We give a polynomial-time algorithm for the constructive microbribery problem. A high-level overview is that we try to find a threshold value $T$ such that there is a microbribery of cost at most $k$ that transforms $E$ into $E^{\prime}$ such that (a) $p$ has $\operatorname{score}_{E^{\prime}}^{\alpha}$ exactly $T$, and (b) every other candidate has score $_{E^{\prime}}^{\alpha}$ at most $T$.

Let $B$ be a number that is greater than the cost of any possible microbribery within $E$ (e.g., $\left.B=\|V\| \cdot\|C\|^{2}+1\right)$. For each possible threshold $T$, we consider a min-cost-flow instance $I(T)$ with node set $K=C \cup\{s, t\}$, where $s$ is the source and $t$ is the sink, the edge capacities and costs are specified in Figure 1, and the target flow value is

$$
F=\sum_{c_{i} \in C} \operatorname{score}_{E}^{\alpha}\left(c_{i}\right)=\frac{\|C\|(\|C\|-1)}{2} .
$$


Voter 1: $\quad c_{0}>c_{1}>c_{2}>c_{3}$

Voter 2: $\quad c_{3}>c_{2}>c_{1}>c_{0}$

\begin{tabular}{|c|rrrr|}
\hline $\operatorname{vs}_{E}\left(c_{i}, c_{j}\right)$ & $c_{0}$ & $c_{1}$ & \multicolumn{1}{c|}{$c_{2}$} & \multicolumn{1}{c|}{$c_{3}$} \\
\hline$c_{0}$ & 0 & 1 & -1 & 1 \\
$c_{1}$ & -1 & 0 & -1 & -1 \\
$c_{2}$ & 1 & 1 & 0 & 1 \\
$c_{3}$ & -1 & 1 & -1 & 0 \\
\hline
\end{tabular}

Figure 2: Sample election $E$ for Example 3.12 in the proof of Lemma 3.11.

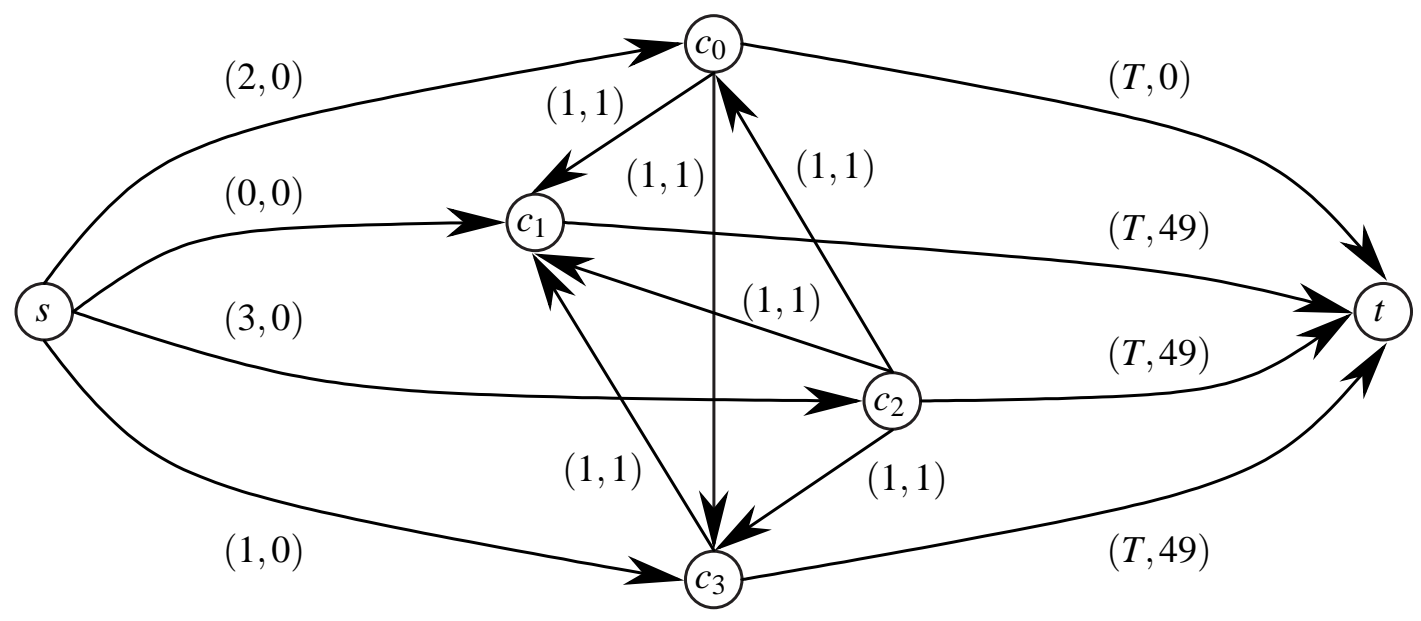

Figure 3: Flow network $I(T)$ corresponding to the instance $\left(E, c_{0}, k\right)$ of Example 3.12.

Example 3.12 For illustration, consider the following example. Suppose the given election $E$ has four candidates and three voters, and the preference tables of the voters (who each happen to be rational in this example) can be obtained from their preference orders that are shown in Figure 2, which also gives the corresponding values of $\operatorname{vs}_{E}\left(c_{i}, c_{j}\right)$ for each pair of candidates. Thus we have $\operatorname{score}_{E}^{\alpha}\left(c_{0}\right)=2$, $\operatorname{score}_{E}^{\alpha}\left(c_{1}\right)=0$, score $E_{E}^{\alpha}\left(c_{2}\right)=3$, and score $_{E}^{\alpha}\left(c_{3}\right)=1$. Suppose further that we are allowed to perform one microbribe, so $k=1$. Clearly, one microbribe that changes the preference of the third voter from $c_{2}>c_{0}$ to $c_{0}>c_{2}$ will flip the outcome of their head-to-head contest from $c_{2}$ winning to $c_{0}$ winning, which is enough to reach our goal of making $c_{0}$ win the election, and this is of course the cheapest possible successful microbribery. Finally, note that in this example we have $B=49$.

For each threshold $T$ with $0 \leq T \leq 3$, the flow network $I(T)$ corresponding to this instance $\left(E, c_{0}, k\right)$ of the constructive microbribery problem is shown in Figure 3, and we have a target flow value of $F=6$. Every edge $e$ in this flow network is labeled by the pair $(c(e), a(e))$ of numbers that give the capacity and the cost of edge e, respectively.

To continue the proof of Lemma 3.11, note that with an odd number of voters, constructive microbribery in Copeland $\mathrm{Irrational}^{\alpha}$ simply requires us to choose for which pairs of distinct candidates we want to flip the outcome of their head-to-head contest in order to ensure $p$ 's victory. Thus it is sufficient to represent a microbribery $M$ as a collection of pairs $\left(c_{i}, c_{j}\right)$ of distinct candidates for whom we need to flip the result of their head-to-head contest from $c_{i}$ winning to $c_{j}$ winning. Clearly, 
given such a collection $M$, the cheapest way to implement it costs

$$
\sum_{\left(c_{i}, c_{j}\right) \in M} \operatorname{wincost}_{E}\left(c_{j}, c_{i}\right)
$$

A crucial observation for our algorithm is that we can directly translate flows to microbriberies using the following interpretation. Let $f$ be a flow (as per Definition 3.8 with all edge flows being integers) of value $F$ within instance $I(T)$. The units of flow that travel through the network correspond to Copeland $\alpha_{\text {Irrational }}^{\alpha}$ points. For each $c_{i} \in C$, we interpret the amount of flow that goes directly from $s$ to $c_{i}$ as the number of Copeland ${ }_{\text {Irrational }}^{\alpha}$ points that $c_{i}$ has before any microbribery is attempted, ${ }^{13}$ and the amount of flow that goes directly from $c_{i}$ to $t$ as the number of Copeland $d_{\text {Irrational }}^{\alpha}$ points that $c_{i}$ has after the microbribery (defined by the flow). The units of flow that travel between distinct $c_{i}$ 's (i.e., through edges of the form $\left(c_{i}, c_{j}\right), i \neq j$ ) correspond to the microbribes exerted: A unit of flow traveling from node $c_{i}$ to $c_{j}$ corresponds to changing the result of the head-to-head contest between $c_{i}$ and $c_{j}$ from $c_{i}$ winning to $c_{j}$ winning. In this case, the Copeland $\mathrm{Irrational}^{\alpha}$ point moves from $c_{i}$ to $c_{j}$ and the cost of the flow increases by $a\left(c_{i}, c_{j}\right)=\operatorname{wincost}\left(c_{j}, c_{i}\right)$, exactly the minimum cost of a microbribery that flips this contest's result. Let $M_{f}$ be the microbribery defined, as just described, by flow $f$. It is easy to see that

$$
\text { flowcost }(f)=B \cdot\left(F-f\left(c_{0}, t\right)\right)+\sum_{\left(c_{i}, c_{j}\right) \in M_{f}} \text { wincost }_{E}\left(c_{j}, c_{i}\right) .
$$

Thus we can easily extract the cost of microbribery $M_{f}$ from the cost of flow $f$.

Our algorithm crucially depends on this correspondence between flows and microbriberies. (Also, in the proofs of Lemmas 3.14 and 3.16 that cover the case of an even number of voters we simply show how to modify the instances $I(T)$ to handle ties, and we show correspondences between the new networks and microbriberies; the rest of these proofs is the same as here.)

Note that for small values of $T$ no flow of value $F$ exists for $I(T)$. The reason for this is that the edges coming into the sink $t$ might not have enough capacity so as to hold a flow of value $F$. In such a situation it is impossible to guarantee that every candidate gets at most $T$ points; there are too many Copeland ${ }_{\text {Irrational }}^{\alpha}$ points to distribute.

Figure 4 gives our algorithm for constructive microbribery in Copeland ${ }_{\text {Irrational }}^{\alpha}$. This algorithm runs in polynomial time since, as we have already mentioned, the min-cost-flow problem is solvable in polynomial time.

Let us now prove that this algorithm is correct. We have presented above how a flow $f$ of value $F$ within the flow network $I(T)$ (with $0 \leq T \leq F$ ) defines a microbribery. Based on this, it is clear that if our algorithm accepts then there is a microbribery of cost at most $k$ that ensures $p$ 's victory.

On the other hand, suppose now that there exists a microbribery of cost at most $k$ that ensures $p$ 's victory in the election. We will show that our algorithm accepts in this case.

Let $M$ be a minimum-cost bribery (of cost at most $k$ ) that ensures $p$ 's victory. As pointed out above, $M$ can be represented as a collection of pairs $\left(c_{i}, c_{j}\right)$ of distinct candidates for whom we flip the result of the head-to-head contest from $c_{i}$ winning to $c_{j}$ winning. The cost of $M$ is

$$
\sum_{\left(c_{i}, c_{j}\right) \in M} \operatorname{wincost}_{E}\left(c_{j}, c_{i}\right)
$$

13. Note that for each $c_{i} \in C$ any flow of value $F$ within $I(T)$ needs to send exactly $\operatorname{score}_{E}^{\alpha}\left(c_{i}\right)$ units from $s$ to $c_{i}$. 


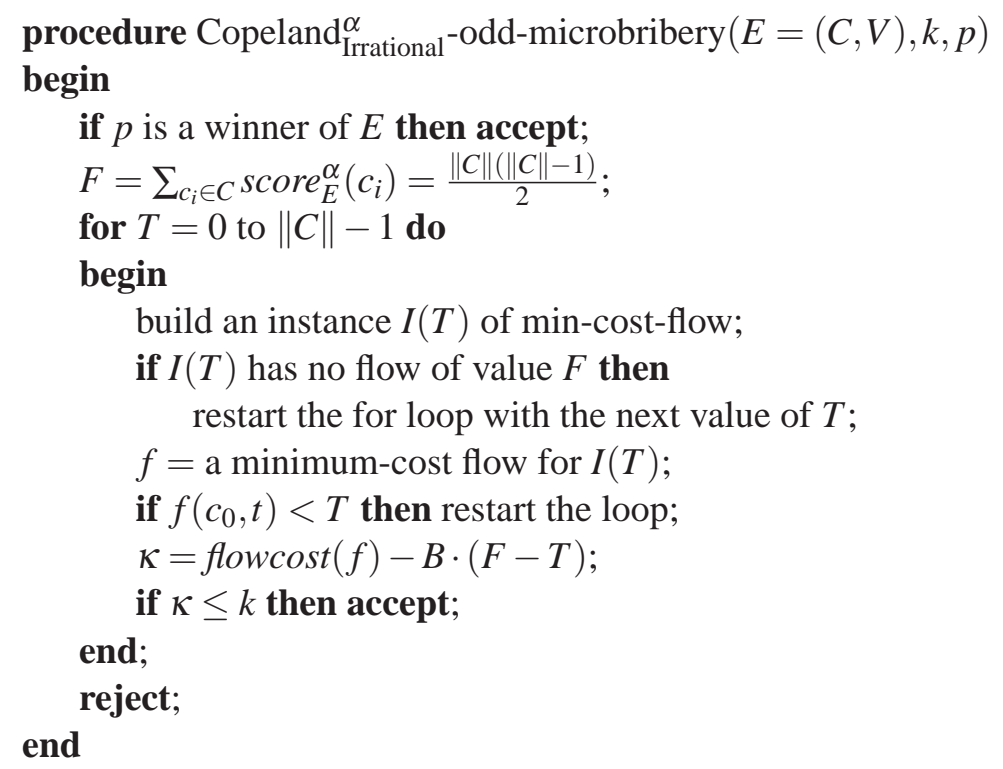

Figure 4: The constructive microbribery algorithm for Copeland ${ }_{\text {Irrational }}^{\alpha}$ elections with an odd number of voters.

Since applying microbribery $M$ ensures that $p$ is a winner, we have that each candidate among $c_{1}, c_{2}, \ldots, c_{m}$ has at most as many Copeland ${ }_{\text {Irrational }}^{\alpha}$ points as $p$ does. Let $E^{\prime}$ be the election that results from $E$ after applying microbribery $M$ to $E$ (i.e., after flipping the results of the contests specified by $M$ in an optimal way, as given by wincost $\left._{E}\right)$. Let $T^{\prime}$ be $\operatorname{score}_{E^{\prime}}^{\alpha}(p), p$ 's Copeland Irrational $^{\alpha}$ score after implementing $M$. Clearly, $0 \leq T^{\prime} \leq\|C\|-1$.

Consider instance $I\left(T^{\prime}\right)$ and let $f_{M}$ be the flow that corresponds to the microbribery $M$. In this flow each edge of the form $\left(s, c_{i}\right)$ carries flow of its maximum capacity, $\operatorname{score}_{E}^{\alpha}\left(c_{i}\right)$, each edge of the form $\left(c_{i}, c_{j}\right)$ carries one unit of flow exactly if $e$ is listed in $M$ and carries zero units of flow otherwise, and each edge of the form $\left(c_{i}, t\right)$ carries $\operatorname{score}_{E^{\prime}}^{\alpha}\left(c_{i}\right)$ units of flow. It is easy to see that this is a legal flow. The cost of $f_{M}$ is

$$
\text { flowcost }\left(f_{M}\right)=B \cdot\left(F-T^{\prime}\right)+\sum_{\left(c_{i}, c_{j}\right) \in M} \operatorname{wincost}_{E}\left(c_{j}, c_{i}\right) .
$$

After applying $M, p$ gets $T^{\prime}$ Copeland ${ }_{\text {Irrational }}^{\alpha}$ points that travel to the sink $t$ via edge $\left(c_{0}, t\right)$ with cost $a\left(c_{0}, t\right)=0$, and all the remaining $F-T^{\prime}$ points travel via edges $\left(c_{i}, t\right), i \in\{1,2, \ldots, m\}$, with cost $a\left(c_{i}, t\right)=B$. The remaining part of flowcost $\left(f_{M}\right)$ is the cost of the units of flow traveling through the edges $\left(c_{i}, c_{j}\right)$ that directly correspond to the cost of microbribery $M$.

Now consider some minimum-cost flow $f_{\min }$ for $I\left(T^{\prime}\right)$. Since $f_{M}$ exists, a minimum-cost flow must exist as well. Clearly, we have

$$
\text { flowcost }\left(f_{\min }\right) \leq \operatorname{flow\operatorname {cost}}\left(f_{M}\right) \text {. }
$$

Let $T^{\prime \prime}$ be the number of units of flow that $f_{\min }$ assigns to travel over the edge $\left(c_{0}, t\right)$, i.e., $T^{\prime \prime}=f_{\min }\left(c_{0}, t\right)$. The only edges with nonzero cost for sending flow through them are those in the 
set $\left\{\left(c_{i}, c_{j}\right) \mid c_{i}, c_{j} \in C \wedge \operatorname{vs}_{E}\left(c_{i}, c_{j}\right)>0\right\} \cup\left\{\left(c_{i}, t\right) \mid i \in\{1, \ldots, m\}\right\}$ and thus the cost of $f_{\min }$ can be expressed as (recall that $\operatorname{vs}_{E}\left(c_{i}, c_{j}\right)>0$ implies $i \neq j$ )

$$
\text { flowcost }\left(f_{\min }\right)=B \cdot\left(F-T^{\prime \prime}\right)+\sum_{c_{i}, c_{j} \in C \wedge \mathrm{vs}_{E}\left(c_{i}, c_{j}\right)>0} f_{\min }\left(c_{i}, c_{j}\right) \cdot \text { wincost }_{E}\left(c_{j}, c_{i}\right) .
$$

It holds that (1) $B>\sum_{i, j, i \neq j}$ wincost $_{E}\left(c_{i}, c_{j}\right)$, (2) for each $c_{i}, c_{j} \in C$ such that $\operatorname{vs}_{E}\left(c_{i}, c_{j}\right)>0$ we have $f_{\min }\left(c_{i}, c_{j}\right) \in\{0,1\}$, and (3) flowcost $\left(f_{\min }\right) \leq \operatorname{flowcost}\left(f_{M}\right)$. So $T^{\prime \prime}=T^{\prime}$ must hold ${ }^{14}$ and it must hold that

$$
\sum_{c_{i}, c_{j} \in C \wedge \mathrm{vs}_{E}\left(c_{i}, c_{j}\right)>0} f_{\min }\left(c_{i}, c_{j}\right) \cdot \text { wincost }_{E}\left(c_{j}, c_{i}\right) \leq \sum_{\left(c_{i}, c_{j}\right) \in M} \text { wincost }_{E}\left(c_{j}, c_{i}\right) .
$$

Thus flow $f_{\min }$ corresponds to a microbribery that guarantees $p$ 's victory and has cost at most as high as that of $M$. Since $M$ was chosen to have minimum cost among all such microbriberies, flow $f_{\min }$ corresponds to a microbribery of minimum cost and our algorithm correctly accepts within the for loop of Figure 4, at the very latest when in the for loop $T$ is set to $T^{\prime}$.

We now turn to the algorithms showing that Llull and Copeland ${ }^{0}$, with irrational voters allowed, are vulnerable to constructive microbribery when the number of voters is even. Here we need to take into account that it sometimes is more desirable to have some candidates tie each other in a head-to-head contest than to have one of them win the contest. We handle the cases of Llull and Copeland ${ }^{0}$ separately, but in each case our proofs follow the same general structure. In each case we first provide a lemma that restricts the set of microbriberies to model, and then we use a slightly modified version of the algorithm from Theorem 3.11, on a modified set of min-cost-flow instances, to solve the thus limited microbribery problem. We omit the proofs of the remaining four lemmas of this section as they are somewhat lengthy and repetitive. However, these proofs can be found in the full TR version of this paper (Faliszewski et al., 2008b).

Lemma 3.13 Let $E=(C, V)$ be an election with candidate set $C=\left\{c_{0}, c_{1}, \ldots, c_{m}\right\}$ and with an even number of voters, specified via preference tables over $C$. If the election is conducted using Copeland $\mathrm{Irrational}^{0}$ then no minimum-cost microbribery that ensures victory for $c_{0}$ involves either (a) flipping a result of a head-to-head contest between any two distinct candidates $c_{i}, c_{j} \in C-\left\{c_{0}\right\}$ from $c_{i}$ winning to $c_{j}$ winning, or $(b)$ changing a result of a head-to-head contest between any two distinct candidates in $C-\left\{c_{0}\right\}$ from a tie to one of them winning.

14. Let us explain why $T^{\prime \prime}=T^{\prime}$. In $I\left(T^{\prime}\right)$, by definition, $c\left(c_{0}, t\right)=T^{\prime}$, so we know that $T^{\prime \prime}=f_{\min }\left(c_{0}, t\right) \leq T^{\prime}$. We will now show that $T^{\prime \prime}=T^{\prime}$. For the sake of contradiction, let us assume that $T^{\prime \prime}<T^{\prime}$. We have

$$
\begin{aligned}
\text { flowcost }\left(f_{\min }\right) & =B \cdot\left(F-T^{\prime \prime}\right)+\sum_{c_{i}, c_{j} \in C \wedge \mathrm{vs}_{E}\left(c_{i}, c_{j}\right)>0} f_{\min }\left(c_{i}, c_{j}\right) \cdot \text { wincost }_{E}\left(c_{j}, c_{i}\right) \\
& \geq B \cdot\left(F-T^{\prime}\right)+B+\sum_{c_{i}, c_{j} \in C \wedge \mathrm{vs}_{E}\left(c_{i}, c_{j}\right)>0} f_{\min }\left(c_{i}, c_{j}\right) \cdot \text { wincost }_{E}\left(c_{j}, c_{i}\right) \\
& >B \cdot\left(F-T^{\prime}\right)+\sum_{\left(c_{i}, c_{j}\right) \in M} \text { wincost }_{E}\left(c_{j}, c_{i}\right) \\
& =\operatorname{flowcost}\left(f_{M}\right),
\end{aligned}
$$

where the last inequality follows from the fact that $B$ is greater than the cost of any microbribery within $E$. We have reached a contradiction, since $f_{\min }$ is a minimum-cost flow in $I\left(T^{\prime}\right)$. Thus $T^{\prime \prime}=T^{\prime}$. 
Lemma 3.14 There is a polynomial-time algorithm that solves the constructive microbribery problem for Copeland $\mathrm{Irrational}^{0}$ elections with an even number of voters (in the nonunique-winner model).

Lemma 3.15 Let $E=(C, V)$ be an election with candidate set $C=\left\{c_{0}, c_{1}, \ldots, c_{m}\right\}$ and with an even number of voters, specified via preference tables over $C$. If the election is conducted using Copeland $\mathrm{Irrational}_{1}^{1}$ then no minimum-cost microbribery that ensures victory for $c_{0}$ involves obtaining a tie in a head-to-head contest between any two distinct candidates in $C-\left\{c_{0}\right\}$.

Lemma 3.16 There is a polynomial-time algorithm that solves the constructive microbribery problem for Copeland $\mathrm{I}_{\text {Irrational }}$ elections with an even number of voters (in the nonunique-winner model).

Together, Theorem 3.7 and Lemmas 3.11, 3.14, and 3.16 show that, in particular, both Copeland $\mathrm{Irrational}^{1}$ and Copeland ${ }_{\text {Irrational }}^{0}$ are vulnerable to microbribery, both in the constructive and the destructive settings. It is interesting to note that all our microbribery proofs would work just as well if we considered a slight twist on the definition of the microbribery problem, namely, if instead of saying that each flip in a voter's preference table has unit cost we would allow each voter to have a possibly different price for flipping each separate entry in his or her preference table. This change would affect only the computing of the values of the functions wincost and tiecost (or, strictly speaking, their analogues in the priced setting). (Technically, we would also have to modify Lemmas 3.13 and 3.15, which in our unit-cost setting say that an optimal microbribery never involves certain specified pairs of candidates, whereas in the priced setting we would need to rephrase them to state that there exist optimal microbriberies that do not involve those specified pairs of candidates.)

An interesting direction for further study of the complexity of bribery within Copeland ${ }^{\alpha}$ systems is to consider a version of the microbribery problem for the case of rational voters. There, one would pay unit cost for a switch of two adjacent candidates on a given voter's preference list.

For Copeland ${ }_{\text {Irrational }}^{\alpha}$, we would also like to know the complexity of constructive microbribery when $\alpha$ is a rational number strictly between 0 and 1 . Our network-flow-based approach does not seem to generalize easily to values of $\alpha$ strictly between 0 and 1 (when the number of voters is even) because in a flow network it is hard to "split" a unit of flow in a tie. A promising approach would be to have several units of flow model one Copeland $\mathrm{Irrational}^{\alpha}$ point (e.g., for the case of $\alpha=\frac{1}{2}$ we could try to use two units of flow to model a single Copeland ${ }^{0.5}$ point), but then it seems very difficult (if not impossible) to find edge costs that appropriately model the microbribery. (It is possible to do so in a very restricted setting, namely where $\alpha=\frac{1}{2}$ and there are exactly two voters that can be bribed.) Also, the results regarding hardness of manipulation of Faliszewski et al. (2008) suggest that microbribery for $\alpha$ strictly between 0 and 1 might be NP-hard. However, again, it is nontrivial to translate their reduction to the world of microbribery.

On a related note, Kern and Paulusma (2001) have shown that the following problem, which they call $\mathrm{SC}(0, \alpha, 1)$, is NP-complete. Let $\alpha$ be a rational number such that $0<\alpha<1$ and $\alpha \neq \frac{1}{2}$. We are given an undirected graph $G=(V(G), E(G))$, where each vertex $u \in V(G)$ is assigned a rational value $c_{u}$ of the form $i+j \alpha$, for nonnegative integers $i$ and $j$. The question, which we have rephrased to state in terms of (a variant of) our notion of Copeland ${ }^{\alpha}$, is whether it is possible to (possibly partially) orient the edges of $G$ such that for each vertex $u \in V(G)$ it holds that $u$ 's Copeland ${ }^{\alpha}$ score is at most $c_{u}$. Here, by "Copeland ${ }^{\alpha}$ score of a vertex $u$ " we mean, as is natural, the number of vertices $u$ "defeats" (i.e., the number of vertices $v$ such that there is a directed edge from $u$ to $v$ ) plus $\alpha$ times the number of vertices that $u$ "ties" with (i.e., the number of vertices such that there is an undirected edge between $u$ and $v$ ). 
Problem $\operatorname{SC}(0, \alpha, 1)$ is very closely related to our microbribery problem. However, we do not see an immediate reduction from $\mathrm{SC}(0, \alpha, 1)$ to microbribery. A natural approach would be to embed graph $G$ into an election (in the sense that will be explored in Section 4) in such a way that our preferred candidate $p$ can become a winner, via a microbribery, if and only if it is possible to orient the edges of $G$ in a way respecting the constraints defined by the values $c_{u}$ (for each $u$ in $V(G)$ ). We would, of course, have to set the budget of our microbribery high enough to allow modifying each of the edges in $G$ and none of the edges outside of $G$. However, this is difficult. The proof of Kern and Paulusma uses values $c_{u}$ that can be implemented only via using tied headto-head contests. The agent performing microbribery could, potentially, affect those head-to-head contests, thus spoiling our reduction.

\section{Control}

In this section we focus on the complexity of control in Copeland ${ }^{\alpha}$ elections. In control problems we are trying to ensure that our preferred candidate $p$ is a winner (or, in the destructive case, that our despised candidate is not a winner) of a given election via affecting this election's structure (namely, via adding, deleting, or partitioning either candidates or voters). In contrast with bribery problems, in control problems we are never allowed to change any of the votes and, consequently, the issues that we encounter and the proof techniques we use are quite different from those presented in the previous section. For the same reason as previously for each standard type of control a resistance result in the rational-voters case implies an analogous resistance result in the irrational-voters case, and a vulnerability result in the irrational-voters case implies an analogous vulnerability result in the rational-voters case.

The literature regarding the complexity of control problems is not large. To the best of our knowledge, the only election systems for which a comprehensive analysis has been conducted previously are plurality, Condorcet, and (variants of) approval voting (see Bartholdi et al., 1992; Hemaspaandra et al., 2007a, 2007b; Betzler and Uhlmann, 2008; Erdélyi et al., 2008b; see also Meir et al., 2008 , for some results on (variants of) approval voting, single nontransferable vote, and cumulative voting with respect to constructive control via adding voters). Among plurality, Condorcet, and (the standard variant of) approval voting, plurality appears to be the least vulnerable to control and so it is natural to compare our new results with those for plurality. However, we mention in passing that Hemaspaandra et al. (2007b) show how to construct hybrid election systems that are resistant to all standard types of control (including both $\mathrm{AC}$ and $\mathrm{AC}_{\mathrm{u}}$; $\mathrm{AC}$ is not discussed or proven in Hemaspaandra et al., 2007b-the " $\mathrm{AC}$ " there is our " $\mathrm{AC}_{\mathrm{u}}$ " - but we mention that the techniques clearly can handle it without any problem). (It should also be noted that these hybrid systems were not designed as "natural" systems to be applied in real-world elections but rather their purpose was to prove a certain impossibility theorem impossible.)

Our main result in this section is Theorem 4.1.

Theorem 4.1 Let $\alpha$ be a rational number with $0 \leq \alpha \leq 1$. Copeland ${ }^{\alpha}$ elections are resistant and vulnerable to control types as indicated in Table 1 in both the nonunique-winner model and the unique-winner model, for both the rational and the irrational voter model.

In particular, we will prove in this section that the notion widely referred to in the literature simply as "Copeland elections," which we here for clarity call Copeland ${ }^{0.5}$, possesses all ten of our basic types (see Table 1) of constructive resistance (and in addition, even has constructive $\mathrm{AC}_{\mathrm{u}}$ 


\begin{tabular}{|l|c|c|c|c|c|c||c|c|}
\hline \multirow{2}{*}{} & \multicolumn{9}{|c||}{ Copeland $^{\alpha}$} & \multicolumn{2}{c|}{ Plurality } \\
\cline { 2 - 7 } & \multicolumn{2}{|c|}{$\alpha=0$} & \multicolumn{2}{c|}{$0<\alpha<1$} & \multicolumn{2}{c|}{$\alpha=1$} & \multicolumn{1}{c|}{} \\
\hline Control type & $\mathrm{CC}$ & $\mathrm{DC}$ & $\mathrm{CC}$ & $\mathrm{DC}$ & $\mathrm{CC}$ & $\mathrm{DC}$ & $\mathrm{CC}$ & $\mathrm{DC}$ \\
\hline $\mathrm{AC}_{\mathrm{u}}$ & $\mathrm{V}$ & $\mathrm{V}$ & $\mathrm{R}$ & $\mathrm{V}$ & $\mathrm{V}$ & $\mathrm{V}$ & $\mathrm{R}$ & $\mathrm{R}$ \\
$\mathrm{AC}$ & $\mathrm{R}$ & $\mathrm{V}$ & $\mathrm{R}$ & $\mathrm{V}$ & $\mathrm{R}$ & $\mathrm{V}$ & $\mathrm{R}$ & $\mathrm{R}$ \\
$\mathrm{DC}$ & $\mathrm{R}$ & $\mathrm{V}$ & $\mathrm{R}$ & $\mathrm{V}$ & $\mathrm{R}$ & $\mathrm{V}$ & $\mathrm{R}$ & $\mathrm{R}$ \\
RPC-TP & $\mathrm{R}$ & $\mathrm{V}$ & $\mathrm{R}$ & $\mathrm{V}$ & $\mathrm{R}$ & $\mathrm{V}$ & $\mathrm{R}$ & $\mathrm{R}$ \\
RPC-TE & $\mathrm{R}$ & $\mathrm{V}$ & $\mathrm{R}$ & $\mathrm{V}$ & $\mathrm{R}$ & $\mathrm{V}$ & $\mathrm{R}$ & $\mathrm{R}$ \\
PC-TP & $\mathrm{R}$ & $\mathrm{V}$ & $\mathrm{R}$ & $\mathrm{V}$ & $\mathrm{R}$ & $\mathrm{V}$ & $\mathrm{R}$ & $\mathrm{R}$ \\
PC-TE & $\mathrm{R}$ & $\mathrm{V}$ & $\mathrm{R}$ & $\mathrm{V}$ & $\mathrm{R}$ & $\mathrm{V}$ & $\mathrm{R}$ & $\mathrm{R}$ \\
\hline PV-TE & $\mathrm{R}$ & $\mathrm{R}$ & $\mathrm{R}$ & $\mathrm{R}$ & $\mathrm{R}$ & $\mathrm{R}$ & $\mathrm{V}$ & $\mathrm{V}$ \\
PV-TP & $\mathrm{R}$ & $\mathrm{R}$ & $\mathrm{R}$ & $\mathrm{R}$ & $\mathrm{R}$ & $\mathrm{R}$ & $\mathrm{R}$ & $\mathrm{R}$ \\
AV & $\mathrm{R}$ & $\mathrm{R}$ & $\mathrm{R}$ & $\mathrm{R}$ & $\mathrm{R}$ & $\mathrm{R}$ & $\mathrm{V}$ & $\mathrm{V}$ \\
DV & $\mathrm{R}$ & $\mathrm{R}$ & $\mathrm{R}$ & $\mathrm{R}$ & $\mathrm{R}$ & $\mathrm{R}$ & $\mathrm{V}$ & $\mathrm{V}$ \\
\hline
\end{tabular}

Table 1: Comparison of control results for Copeland ${ }^{\alpha}$ elections, where $\alpha$ with $0 \leq \alpha \leq 1$ is a rational number, and for plurality-rule elections. $\mathrm{R}$ means resistance to a particular control type and $\mathrm{V}$ means vulnerability. The results regarding plurality are due to Bartholdi et al. (1992) and Hemaspaandra et al. (2007a). (Note that CCAC and DCAC resistance results for plurality, not handled explicitly in Bartholdi et al., 1992, or Hemaspaandra et al., 2007a, follow immediately from the respective $\mathrm{CCAC}_{\mathrm{u}}$ and $\mathrm{DCAC}_{\mathrm{u}}$ results.)

resistance). (As to why we consider $\mathrm{AC}$ more basic than $\mathrm{AC}_{\mathrm{u}}$, see the discussion in the "Control via Adding Candidates" subpart of Section 2.2. Nonetheless, we do obtain $\mathrm{AC}_{\mathrm{u}}$ results, and so fans of the naturalness of $\mathrm{AC}_{\mathrm{u}}$ will know how things fare under that control type.) And we will establish that the other notion that in the literature is occasionally referred to as "Copeland elections," namely Copeland $^{0}$, as well as Llull elections, which are here denoted by Copeland ${ }^{1}$, both possess all ten of our basic types of constructive resistance. However, we will show that Copeland ${ }^{0}$ and Copeland ${ }^{1}$ are vulnerable to an eleventh type of constructive control, the incongruous but historically resonant notion of constructive control by adding an unlimited number of candidates (i.e., $\mathrm{CCAC}_{\mathrm{u}}$ ).

Note that Copeland ${ }^{0.5}$ has a higher number of constructive resistances, by three, than even plurality, which was before this paper the reigning champ among natural election systems with a polynomial-time winner-determination procedure. (Although the results regarding plurality in Table 1 are stated for the unique-winner version of control, for all the table's Copeland ${ }^{\alpha}$ cases, $0 \leq \alpha \leq 1$, our results hold both in the cases of unique winners and of nonunique winners, so that regardless of which of the two winner models one finds more natural, one will know what holds in that model.) Admittedly, plurality does perform better with respect to destructive candidate control problems, but still our study of Copeland ${ }^{\alpha}$ makes significant steps forward in the quest for a fully control-resistant natural election system with an easy winner problem.

Among the systems with a polynomial-time winner problem, Copeland ${ }^{0.5}$ —and indeed all Copeland $^{\alpha}, 0<\alpha<1$-have the most resistances currently known for any natural election system whose voters vote by giving preference lists. We mention that after our work, Erdélyi et al. (2008b) have shown that their variant of a variant of approval voting proposed by Brams and Sanver (2006) - a certain rather subtle election system with a richer voter preference type (each voter 
specifies both a permutation and a set) that combines approval with preference-based voting-has nineteen (out of a possible twenty-two) control resistances.

This section is organized as follows. The next two sections are devoted to proving Theorem 4.1, and Section 4.3 considers the case of control in elections with a bounded number of candidates or voters. In particular, Section 4.1 focuses on the upper part of Table 1 and studies control problems that affect the candidate structure. Section 4.2 is devoted to voter control and covers the lower part of Table 1. Finally, in Section 4.3 we study the fixed-parameter complexity of control problems. In particular, we take the role of someone who tries to solve in-general-resistant control problems and we devise some efficient algorithms for the case where the number of candidates or the number of voters is bounded.

All our resistance results regarding candidate control follow via reductions from vertex cover and all our vulnerability results follow via greedy algorithms. Our resistance results for the case of control by modifying voter structure follow from reductions from the $\mathrm{X} 3 \mathrm{C}$ problem.

\subsection{Candidate Control}

We start our discussion of candidate control for Copeland ${ }^{\alpha}$ with our results on destructive control. It is somewhat disappointing that for each rational $\alpha, 0 \leq \alpha \leq 1$, Copeland ${ }^{\alpha}$ is vulnerable to each type of destructive candidate control. On the positive side, our vulnerability proofs follow via natural greedy algorithms and will allow us to smoothly get into the spirit of candidate-control problems.

\subsubsection{Destructive Candidate Control}

The results for destructive control by adding and deleting candidates use the following observation.

Observation 4.2 Let $(C, V)$ be an election, and let $\alpha$ be a rational number such that $0 \leq \alpha \leq 1$. For every candidate $c \in C$ it holds that

$$
\operatorname{score}_{(C, V)}^{\alpha}(c)=\sum_{d \in C-\{c\}} \operatorname{score}_{(\{c, d\}, V)}^{\alpha}(c) .
$$

Theorem 4.3 For each rational number $\alpha$ with $0 \leq \alpha \leq 1$, Copeland ${ }^{\alpha}$ is vulnerable to destructive control via adding candidates (both limited and unlimited, i.e., DCAC and DCAC $\mathrm{u}_{\mathrm{u}}$ ), in both the nonunique-winner model and the unique-winner model, for both the rational and the irrational voter model.

Proof. Our input is a set $C$ of candidates, a set $D$ of spoiler candidates, a collection $V$ of voters with preferences (either preference lists or preference tables) over $C \cup D$, a candidate $p \in C$, and a nonnegative integer $k$ (for the unlimited version of the problem we let $k=\|D\|$ ). We ask whether there is a subset $D^{\prime}$ of $D$ such that $\left\|D^{\prime}\right\| \leq k$ and $p$ is not a winner (is not a unique winner) of Copeland ${ }^{\alpha}$ election $E^{\prime}=\left(C \cup D^{\prime}, V\right)$. Note that if $k=0$, this amounts to determining whether $p$ is not a winner (is not a unique winner) of election $E$, which can easily be done in polynomial time.

For the remainder of this proof we will assume that $k>0$. Let $c$ be any candidate in $(C \cup D)-$ $\{p\}$. We define $a(c)$ to be the maximum value of the expression

$$
\operatorname{score}_{\left(C \cup D^{\prime}, V\right)}^{\alpha}(c)-\operatorname{score}_{\left(C \cup D^{\prime}, V\right)}^{\alpha}(p)
$$


under the conditions that $D^{\prime} \subseteq D, c \in C \cup D^{\prime}$, and $\left\|D^{\prime}\right\| \leq k$. From Observation 4.2 it follows that $a(c)$ is the maximum value of

$$
\operatorname{score}_{(C \cup\{c\}, V)}^{\alpha}(c)-\operatorname{score}_{(C \cup\{c\}, V)}^{\alpha}(p)+\sum_{d \in D^{\prime}-\{c\}}\left(\operatorname{score}_{(\{c, d\}, V)}^{\alpha}(c)-\operatorname{score}_{(\{p, d\}, V)}^{\alpha}(p)\right)
$$

under the conditions that $D^{\prime} \subseteq D, c \in C \cup D^{\prime}$, and $\left\|D^{\prime}\right\| \leq k$.

Clearly, $p$ can be prevented from being a winner (a unique winner) if and only if there exists a candidate $c \in(C \cup D)-\{p\}$ such that $a(c)>0$ (such that $a(c) \geq 0$ ).

Given a candidate $c \in(C \cup D)-\{p\}$, it is easy to construct in polynomial time a set $D^{\prime} \subseteq D$, $\left\|D^{\prime}\right\| \leq k$, that yields the value $a(c)$. We start with $D^{\prime}=\emptyset$. If $c \in D$, we add $c$ to $D^{\prime}$. Then we add those candidates $d \in D-D^{\prime}$ to $D^{\prime}$ such that $\operatorname{score}_{(\{c, d\}, V)}^{\alpha}(c)-\operatorname{score}_{(\{p, d\}, V)}^{\alpha}(p)$ is positive, starting with those for whom this value is highest, until $\left\|D^{\prime}\right\|=k$ or no more such candidates exist.

Theorem 4.4 For each rational number $\alpha$ with $0 \leq \alpha \leq 1$, Copeland ${ }^{\alpha}$ is vulnerable to destructive control via deleting candidates $(D C D C)$, in both the nonunique-winner model and the uniquewinner model, for both the rational and the irrational voter model.

The proof of Theorem 4.4 is similar to that of Theorem 4.3, so we do not include it here but instead refer to the full TR version (Faliszewski et al., 2008b).

Destructive control via partitioning of candidates (with or without run-off) is also easy. Since the arguments of that proof are more involved, we present it here.

Theorem 4.5 For each rational number $\alpha$ with $0 \leq \alpha \leq 1$, Copeland ${ }^{\alpha}$ is vulnerable to destructive control via partitioning of candidates and via partitioning of candidates with run-off (in both the TP and TE model, i.e., DCPC-TP, DCPC-TE, DCRPC-TP, and DCRPC-TE), in both the nonuniquewinner model and the unique-winner model, for both the rational and the irrational voter model.

Proof. It is easy to see that in the TP model, $p$ can be prevented from being a winner via partitioning of candidates (with or without run-off) if and only if there is a set $C^{\prime} \subseteq C$ such that $p \in C^{\prime}$ and $p$ is not a winner of $\left(C^{\prime}, V\right)$. It follows that $p$ can be prevented from being a winner if and only if $p$ can be prevented from being a winner by deleting at most $\|C\|-1$ candidates, which can be determined in polynomial time by Theorem 4.4. We will show how to handle the unique-winner variants of DCPC-TP and DCRPC-TP later in this proof.

For the TE model, it is easy to see that if there is a set $C^{\prime} \subseteq C$ such that $p \in C^{\prime}$ and $p$ is not a unique winner of $\left(C^{\prime}, V\right)$ then $p$ can be prevented from being a unique winner via partitioning of candidates (with or without run-off). One simply partitions the candidates into $C^{\prime}$ and $C-C^{\prime}$ and thus $p$ fails to advance to the final stage. On the other hand, if $p$ can be prevented from being a winner (a unique winner) via partitioning of candidates (with or without run-off) in the TE model, then there exists a set $C^{\prime} \subseteq C$ such that $p \in C^{\prime}$ and $p$ is not a unique winner of $\left(C^{\prime}, V\right)$. This is so because then either $p$ does not advance to the final stage (and this means that $p$ is not a unique winner of his or her first-stage election) or $p$ is not a winner (not a unique winner) of the final stage (note that not being a winner implies not being a unique winner).

Thus, $p$ can be prevented from being a winner (a unique winner) via partitioning of candidates (with or without run-off) in the TE model if and only if there is a set $C^{\prime} \subseteq C$ such that $p \in C^{\prime}$ and $p$ 
is not a unique winner of $\left(C^{\prime}, V\right)$. Clearly, such a set exists if and only if $p$ can be prevented from being a unique winner via deleting at most $\|C\|-1$ candidates, which by Theorem 4.4 can be tested in polynomial time.

It remains to show that Copeland ${ }^{\alpha}$ is vulnerable to destructive control via partitioning of candidates (with or without run-off), in both the rational and the irrational voter model, in the uniquewinner model with the TP tie-handling rule. In the argument below we focus on the DCRPC-TP case but it is easy to see that essentially the same reasoning works for DCPC-TP.

First we determine whether $p$ can be precluded from being a winner in our current control scenario. This can be done in polynomial time as explained above. If $p$ can be precluded from being a winner, $p$ can certainly be precluded from being a unique winner, and we are done. For the remainder of the proof, suppose that $p$ cannot be precluded from being a winner in our current control scenario, i.e., for every set $D \subseteq C$ such that $p \in D, p$ is a winner of $(D, V)$. Let

$$
D_{1}=\{c \in C-\{p\} \mid p \text { defeats } c \text { in a head-to-head contest }\}
$$

and let $D_{2}=D-\left(D_{1} \cup\{p\}\right)$. Note that for all $c \in D_{2}, p$ ties $c$ in a head-to-head contest, since otherwise $p$ would not be a winner of $(\{c, p\}, V)$. If $D_{2}=\emptyset$, then $p$ is a Condorcet winner and no partition (with or without run-off) can prevent $p$ from being a unique winner (Hemaspaandra et al., 2007a). For the remainder of the proof, we assume that $D_{2} \neq \emptyset$. We will show that $p$ can be precluded from being a unique winner in our current control scenario.

If $\alpha<1$, we let the first subelection be $\left(D_{1} \cup\{p\}, V\right)$. Note that $p$ is the unique winner of this subelection. The final stage of the election involves $p$ and one or more candidates from $D_{2}$. Note that every pair of candidates in $D_{2} \cup\{p\}$ is tied in a head-to-head election (since if $c$ would defeat $d$ in a head-to-head election, $c$ would be the unique winner of $(\{c, d, p\}, V)$, which contradicts the assumption that $p$ is a winner of every subelection it participates in). It follows that all candidates that participate in the final stage of the election are winners, and so $p$ is not a unique winner.

Finally, consider the case that $\alpha=1$. Then $\operatorname{score}_{(C, V)}^{\alpha}(p)=\|C\|-1$. If there is a candidate $d \in C-\{p\}$ such that $\operatorname{score}_{(C, V)}^{\alpha}(d)=\|C\|-1$, then $d$ will always (i.e., in every subelection containing $d$ ) be a winner, and thus $p$ will not be a unique winner of the final stage of the election, regardless of which partition of $C$ was chosen. Now suppose that $\operatorname{score}_{(C, V)}^{\alpha}(d)<\|C\|-1$ for all $d \in C-\{p\}$. Then $\operatorname{score}_{(C, V)}^{\alpha}(d) \leq\|C\|-2$ for all $d \in C-\{p\}$. Let $c$ be a candidate in $D_{2}$ and let the first subelection be $(C-\{c\}, V)$. Let $C^{\prime}$ be the set of winners of $(C-\{c\}, V)$. Since $\operatorname{score}_{(C-\{c\}, V)}^{\alpha}(p)=\|C\|-2$, it holds that $p \in C^{\prime}$ and for every $d \in C^{\prime}-\{p\}, \operatorname{score}_{(C-\{c\}, V)}^{\alpha}(d)=$ $\|C\|-2$. Since score $_{(C, V)}^{\alpha}(d) \leq\|C\|-2$, it follows that $c$ defeats $d$ in a head-to-head election. The final stage of the election involves candidates $C^{\prime} \cup\{c\}$. Note that $\operatorname{score}_{\left(C^{\prime} \cup\{c\}, V\right)}^{\alpha}(c)=\left\|C^{\prime}\right\|$, and thus $c$ is a winner of the election, and we have precluded $p$ from being a unique winner.

The above vulnerability results for the case of destructive candidate control should be contrasted with the essentially perfect resistance to constructive candidate control (with the exception of constructive control via adding an unlimited number of candidates for Copeland ${ }^{\alpha}$ with $\alpha \in\{0,1\}$ ) that will be shown in Section 4.1.3. But first, in Section 4.1.2, we will provide some technical prerequisites. 


\subsubsection{COnstructing Instances of Elections}

Many of our proofs in the next section require constructing fairly involved instances of Copeland ${ }^{\alpha}$ elections. In this section we provide several lemmas and observations that simplify building such instances.

We first note that each election $E=(C, V)$ induces a directed graph $G(E)$ whose vertices are $E$ 's candidates and whose edges correspond to the results of the head-to-head contests in $E$. That is, for each two distinct vertices of $G(E)$ (i.e., for each two distinct candidates), $a$ and $b$, there is an edge from $a$ to $b$ if and only if $a$ defeats $b$ in their head-to-head contest (i.e., if and only if $\operatorname{vs}_{E}(a, b)>0$ ). Clearly, $G(E)$ does not depend on the value of $\alpha$. The following fundamental result is due to McGarvey. This result allows us to basically identify elections with their election graphs in the proofs of resistance for candidate control. In effect, Copeland ${ }^{\alpha}$ candidate-control problems often can be viewed (with some care regarding ties) as graph-theoretic problems.

Lemma 4.6 (McGarvey, 1953) There is a polynomial-time algorithm that given as input an antisymmetric directed graph $G$ outputs an election $E$ such that $G=G(E)$.

Proof. For the sake of completeness, we give the algorithm. Let $G$ be an antisymmetric directed graph. The algorithm computes the election $E=(C, V)$, where $C=V(G)$ and for each edge $(a, b)$ in $G$ there are exactly two voters, one with preference list $a>b>C-\{a, b\}$ and one with preference list $\overleftarrow{C-\{a, b\}}>a>b$. Since $G$ is antisymmetric, it is easy to see that $G=G(E)$.

The above basic construction of McGarvey was improved upon by Stearns (1959). While McGarvey's construction requires twice as many voters as there are edges in $G$, the construction of Stearns needs at most $\|V(G)\|+2$ voters. Stearns also provides a lower bound on the number of voters that are needed to represent an arbitrary graph via an election. (It is easy to see that any such graph can be modeled via two irrational voters but the lower bound for the case of rational votes is somewhat harder.)

We will often construct complicated elections via combining simpler ones (see, in particular, the rather involved proofs of Theorems 4.12 through 4.16 that can be found in the full TR version, Faliszewski et al., 2008b). Whenever we speak of combining two elections, say $E_{1}=\left(C_{1}, V_{1}\right)$ and $E_{2}=\left(C_{2}, V_{2}\right)$, we mean building, via the algorithm from Lemma 4.6, an election $E=(C, V)$ whose election graph is a disjoint union of the election graphs of $E_{1}$ and $E_{2}$ with, possibly, some edges added between the vertices of $G\left(E_{1}\right)$ and $G\left(E_{2}\right)$ (in each case we will explicitly state which edges, if any, are added). In particular, we will often want to add some padding candidates to an election, without affecting the original election much. In order to do so, we will typically combine our main election with one of the following "padding" elections. Note that this construction, which we originally developed for use in the study of control for Copeland ${ }^{\alpha}$ voting, has also proven useful in the study of manipulation for Copeland ${ }^{\alpha}$ (Faliszewski et al., 2008).

Lemma 4.7 Let $\alpha$ be a rational number such that $0 \leq \alpha \leq 1$. For each positive integer $n$, there is a polynomial-time (in $n$ ) computable election $\operatorname{Pad}_{n}=(C, V)$ such that $\|C\|=2 n+1$ and for each candidate $c_{i} \in C$ it holds that $\operatorname{score}_{\mathrm{Pad}_{n}}^{\alpha}(c)=n$.

Proof. Fix a positive integer $n$. By Lemma 4.6 it is enough to construct (in polynomial time in $n$ ) a directed, antisymmetric graph $G$ with $2 n+1$ vertices, each with its indegree and outdegree equal 
to $n$. We set $G$ 's vertex set to be $\{0,1, \ldots, 2 n\}$ and we put an edge from vertex $i$ to vertex $j(i \neq j)$ if and only if $(j-i) \bmod (2 n+1) \leq n$. As a result there is exactly one directed edge between every two distinct vertices and for each vertex $i$ we have edges going out from $i$ to exactly the vertices $(i+1) \bmod (2 n+1),(i+2) \bmod (2 n+1), \ldots,(i+n) \bmod (2 n+1)$. Thus, both the indegree and the outdegree of each vertex is equal to $n$ and the proof is complete.

Lemma 4.6 (McGarvey, 1953) is very useful when building an election in which we need direct control over the results of all head-to-head contests. However, in many cases explicitly specifying the results of all head-to-head contests would be very tedious. Instead it would be easier to specify the results of only the important head-to-head contests and require all candidates to have certain suitable scores. In the next lemma we show how to construct elections specified in such a way via combining a "small" election containing the important head-to-head contest with a "large" padding election. We mention that a generalized version of this lemma has since been used to study manipulation for Copeland ${ }^{\alpha}$ (Faliszewski et al., 2008).

Lemma 4.8 Let $E=(C, V)$ be an election where $C=\left\{c_{1}, \ldots, c_{n^{\prime}}\right\}$, let $\alpha$ be a rational number such that $0 \leq \alpha \leq 1$, and let $n \geq n^{\prime}$ be an integer. For each candidate $c_{i}$ we denote the number of headto-head ties of $c_{i}$ in $E$ by $t_{i}$. Let $k_{1}, \ldots, k_{n^{\prime}}$ be a sequence of $n^{\prime}$ nonnegative integers such that for each $k_{i}$ we have $0 \leq k_{i} \leq n$. There is an algorithm that in polynomial time in $n$ outputs an election $E^{\prime}=\left(C^{\prime}, V^{\prime}\right)$ such that:

1. $C^{\prime}=C \cup D$, where $D=\left\{d_{1}, \ldots, d_{2 n^{2}}\right\}$,

2. $E^{\prime}$ restricted to $C$ is $E$,

3. the only ties in head-to-head contests in $E^{\prime}$ are between candidates in $C$,

4. for each $i, 1 \leq i \leq n^{\prime}$, $\operatorname{score}_{E^{\prime}}^{\alpha}\left(c_{i}\right)=2 n^{2}-k_{i}+t_{i} \alpha$, and

5. for each $i, 1 \leq i \leq 2 n^{2}$, score $_{E^{\prime}}^{\alpha}\left(d_{i}\right) \leq n^{2}+1$.

Proof. We build $E^{\prime}$ via combining $E$ with a padding election $F$ (see Lemma 4.7 and the paragraph just before it). $F=(D, W)$, where $D=\left\{d_{1}, \ldots, d_{2 n^{2}}\right\}$, is essentially the election $\operatorname{Pad}_{n^{2}}$ with one arbitrary candidate removed. We partition the candidates in $D$ into $n$ groups, $D_{1}, \ldots, D_{n}$, each with exactly $2 n$ candidates and we set the results of head-to-head contests between each $c_{i} \in C$ and the candidates in $D$ according to the following scheme. For each $j \in\left\{1, \ldots, n^{\prime}\right\}$ such that $i \neq j, c_{i}$ defeats all members of $D_{j}$ and $c_{i}$ defeats exactly as many candidates in $D_{i}$ (and loses to all the remaining ones) as needed to ensure that

$$
\operatorname{score}_{E^{\prime}}^{\alpha}\left(c_{i}\right)=2 n^{2}-k_{i}+t_{i} \alpha .
$$

It is easy to see that this is possible: $c_{i}$ 's score in $\left(C^{\prime}-D_{i}, V^{\prime}\right)$ is $2 n^{2}-2 n+k^{\prime}+t_{i} \alpha$ for some $k^{\prime}$ such that $0 \leq k^{\prime} \leq n^{\prime}-t_{i}$. There are $2 n$ candidates in $D_{i}$ and so $c_{i}$ can reach any score of the form $2 n^{2}-k+t_{i} \alpha$, where $k$ is an integer between 0 and $n$, via defeating in head-to-head contests an appropriate number of candidates in $D_{i}$ and losing to all the remaining ones.

Finally, since $F$ is $\operatorname{Pad}_{n^{2}}$ with one candidate removed, each $d_{i}$ gets at most $n^{2}$ points from defeating other members of $D$ and at most one point from possibly defeating some member of $C$. Thus, for each $d_{i} \in D$, it holds that $\operatorname{score}_{E^{\prime}}^{\alpha}\left(d_{i}\right) \leq n^{2}+1$. This completes the proof. 
Instead of invoking Lemma 4.8 directly, we will often simply describe an election in terms of the results of important head-to-head contests and the scores of the important candidates and then mention that such an election can be built, possibly with adding extra padding candidates that do not affect the general structure of the election, using Lemma 4.8. In each such case it will be clear that Lemma 4.8 can indeed be used to build the election we describe.

\subsubsection{Constructive Candidate Control}

Let us now turn to the case of constructive candidate control. Here we show that resistance holds for Copeland ${ }^{\alpha}$ in all cases (i.e., for all rational values of $\alpha$ with $0 \leq \alpha \leq 1$ and for all constructive candidate control scenarios), except for $\mathrm{CCAC}_{\mathrm{u}}$ for $\alpha \in\{0,1\}$ where vulnerability holds (see Theorem 4.11).

All our resistance proofs in this section follow via reductions from the vertex cover problem. Recall that in the vertex cover problem our input is $(G, k)$ where $G$ is an undirected graph and $k$ a nonnegative integer and we accept if and only if $G$ has a vertex cover of size at most $k$. Without the loss of generality, we assume that $V(G)=\{1, \ldots, n\}$ and $E(G)=\left\{e_{1}, \ldots, e_{m}\right\}$. Note that if either $m=0, n=0$, or $k \geq \min (n, m)$ then the instance has a trivial solution and so in our proofs we will always assume that both $n$ and $m$ are nonzero and that $k$ is less than $\min (n, m)$. In each case, if the input to our reduction does not meet these requirements (or is otherwise malformed) the reduction outputs a fixed "yes" instance or a fixed "no" instance depending on the (easily obtained) solution to $(G, k)$ or the malformation of the input. Also note that for every input $(G, k)$ that meets our requirements, $G$ has a vertex cover of size less than or equal to $k$ if and only if $G$ has a vertex cover of size $k$.

Theorem 4.9 Let $\alpha$ be a rational number such that $0 \leq \alpha \leq 1$. Copeland ${ }^{\alpha}$ is resistant to constructive control via adding candidates (CCAC), in both the nonunique-winner model and the uniquewinner model, for both the rational and the irrational voter model.

Proof. We give a reduction from the vertex cover problem. Let $(G, k)$ be an instance of the vertex cover problem, where $G$ is an undirected graph, $k$ is a nonnegative integer, $V(G)=\{1, \ldots, n\}$, $E(G)=\left\{e_{1}, \ldots, e_{m}\right\}, n \neq 0, m \neq 0$, and $k<\min (n, m)$. We construct an instance of CCAC for Copeland $^{\alpha}$ such that a designated candidate $p$ can become a winner after adding at most $k$ candidates if and only if $G$ has a vertex cover of size at most $k$.

Our reduction works as follows. Via Lemma 4.8, we build an election $E^{\prime}=\left(C^{\prime}, V^{\prime}\right)$ such that:

1. $\left\{p, e_{1}, \ldots, e_{m}\right\} \subseteq C^{\prime}$,

2. $\operatorname{score}_{E^{\prime}}^{\alpha}(p)=2 \ell^{2}-1$ in the nonunique-winner case $\left(\operatorname{score}_{E^{\prime}}^{\alpha}(p)=2 \ell^{2}\right.$ in the unique-winner case); $\ell$ is a sufficiently large (but polynomially bounded) integer that takes the role of Lemma 4.8's $n$,

3. for each $e_{i} \in C^{\prime}$, $\operatorname{score}_{E^{\prime}}^{\alpha}\left(e_{i}\right)=2 \ell^{2}$, and

4. the scores of all candidates in $C^{\prime}-\left\{p, e_{1}, \ldots, e_{m}\right\}$ are at most $2 \ell^{2}-n-2$.

We form election $E=(C, V)$ by combining $E^{\prime}$ with candidates $D=\{1, \ldots, n\}$ (corresponding to the vertices of $G$ ). The results of the head-to-head contests within $D$ are set arbitrarily, and the head-to-head contests between the members of $C$ and the members of $D$ are set as follows: All 
candidates in $C-\left\{e_{1}, \ldots, e_{m}\right\}$ defeat all members of $D$, and for each $i \in D$ and each $e_{j} \in\left\{e_{1}, \ldots, e_{m}\right\}$, candidate $i$ defeats $e_{j}$ if $e_{j}$ is an edge incident to $i$ and loses otherwise. Our reduction outputs an instance $(C, D, V, p, k)$ of CCAC and the question is whether it is possible to choose a subset $D^{\prime} \subseteq D$, $\left\|D^{\prime}\right\| \leq k$, such that $p$ is a winner (the unique winner) of Copeland ${ }^{\alpha}$ election $\left(C \cup D^{\prime}, V\right)$. It is clear that this reduction is computable in polynomial time. We will now show that it is correct.

If $G$ does have a vertex cover of size $k$ then add the candidates in $D$ that correspond to the cover. Adding these candidates increases the score of $p$ by $k$, while the scores of the $e_{i}$ 's can increase only by $k-1$ each, since each edge is incident with at least one member of the vertex cover. Clearly, candidates in $C-\left\{p, e_{1}, \ldots, e_{m}\right\}$ can never become winners by adding at most $k$ candidates from $D$, and thus $p$ becomes a winner (the unique winner).

For the converse, assume that $p$ can become a winner (the unique winner) via adding at most $k$ candidates from the set $D$. In order for $p$ to become a winner (the unique winner), it must be the case that via adding candidates each $e_{i}$ gets at least one point less than $p$. However, this is possible only if we add candidates that correspond to a cover.

Interestingly, when the parameter $\alpha$ is strictly between 0 and 1 (i.e., $0<\alpha<1$ ) then Copeland ${ }^{\alpha}$ is resistant to constructive control via adding candidates even if we allow adding an unlimited number of candidates (the $\mathrm{CCAC}_{\mathrm{u}}$ case). The reason for this is that for each rational $\alpha$ strictly between 0 and 1 our construction will ensure, via its structure, that we can add at most $k$ candidates. On the other hand, both Copeland ${ }^{0}$ and Copeland ${ }^{1}$ are vulnerable to constructive control via adding an unlimited number of candidates $\left(\mathrm{CCAC}_{\mathrm{u}}\right.$, see Theorem 4.11).

Theorem 4.10 Let $\alpha$ be a rational number such that $0<\alpha<1$. Copeland ${ }^{\alpha}$ is resistant to constructive control via adding an unlimited number of candidates $\left(C C A C_{\mathrm{u}}\right)$, in both the nonunique-winner model and the unique-winner model, for both the rational and the irrational voter model.

Proof. We give a reduction from the vertex cover problem.

For the unique-winner case, we will need to specify one of the candidates' scores in terms of a number $\varepsilon>0$ such that $1-\varepsilon \geq \alpha$. Let $t_{1}$ and $t_{2}$ be two positive integers such that $\alpha=\frac{t_{1}}{t_{2}}$ and such that their greatest common divisor is 1. Clearly, two such numbers exist because $\alpha$ is rational and greater than 0 . We set $\varepsilon$ to be $\frac{1}{t_{2}}$. By elementary number-theoretic arguments, there are two positive integer constants, $k_{1}$ and $k_{2}$, such that $k_{1} \alpha=k_{2}-\varepsilon$.

Let $(G, k)$ be an instance of the vertex cover problem, where $G$ is an undirected graph and $k$ is a nonnegative integer. Let $\left\{e_{1}, \ldots, e_{m}\right\}$ be $G$ 's edges and let $\{1, \ldots, n\}$ be $G$ 's vertices. As before, we assume that both $n$ and $m$ are nonzero and that $k<\min (n, m)$. Using Lemma 4.8, we can build an election $E^{\prime}=\left(C, V^{\prime}\right)$ with the following properties:

1. $\left\{p, r, e_{1}, \ldots, e_{m}\right\} \subseteq C$ (the remaining candidates in $C$ are used for padding),

2. $\operatorname{score}_{E^{\prime}}^{\alpha}(p)=2 \ell^{2}-1$,

3. $\operatorname{score}_{E^{\prime}}^{\alpha}(r)=2 \ell^{2}-1-k+k \alpha$ in the nonunique-winner case $\left(\operatorname{score}_{E^{\prime}}^{\alpha}(r)=2 \ell^{2}-1-k+k \alpha-\varepsilon\right.$ in the unique-winner case ${ }^{15}$ ),

15. Note that via the second paragraph of the proof it is easy to build an election where $r$ has a score of this form. To obtain the $-\varepsilon$ part of $r$ 's score we could, for example, have $r$ tie with $k_{1}$ padding candidates to obtain $k_{2}-\varepsilon$ points. The $k_{2}$ points could be accounted for as part of $2 \ell^{2}-1$. 
4. for each $e_{i} \in C$, $\operatorname{score}_{E^{\prime}}^{\alpha}\left(e_{i}\right)=2 \ell^{2}-1+\alpha$ in the nonunique-winner case $\left(\operatorname{score}_{E^{\prime}}^{\alpha}\left(e_{i}\right)=2 \ell^{2}-1\right.$ in the unique-winner case), and

5. the scores of all candidates in $C-\left\{p, r, e_{1}, \ldots, e_{m}\right\}$ are at most $2 \ell^{2}-n-2$.

We form election $E=(C \cup D, V)$ via combining $E^{\prime}$ with candidates $D=\{1, \ldots, n\}$ and appropriate voters such that the results of the head-to-head contests are:

1. $p$ ties with all candidates in $D$,

2. for each $e_{j}$, if $e_{j}$ is incident with some $i \in D$ then candidate $i$ defeats candidate $e_{j}$, and otherwise they tie, and

3. all other candidates in $C$ defeat each of the candidates in $D$.

We will now show that $G$ contains a vertex cover of size at most $k$ if and only if there is a set $D^{\prime} \subseteq D$ such that $p$ is a winner (the unique winner) of Copeland ${ }^{\alpha}$ election $\left(C \cup D^{\prime}, V\right)$. It is easy to see that if $D^{\prime}$ corresponds to a vertex cover of size at most $k$ then $p$ is a winner (the unique winner) of Copeland ${ }^{\alpha}$ election $\left(C \cup D^{\prime}, V\right)$. The reason is that adding any member of $D^{\prime}$ increases $p$ 's score by $\alpha$ and increases $r$ 's score by one, and for each $e_{j}$, adding $i \in D^{\prime}$ increases $e_{j}$ 's score by $\alpha$ if and only if $e_{j}$ is not incident with $i$. Thus, via a simple calculation of the scores of the candidates, it is easy to see that $p$ is a winner (the unique winner) of this election.

On the other hand, assume that $p$ can become a winner (the unique winner) of Copeland ${ }^{\alpha}$ election $\left(C \cup D^{\prime}, V\right)$ via adding some subset $D^{\prime}$ of candidates from $D$. First, note that $\left\|D^{\prime}\right\| \leq k$, since otherwise $r$ would end up with more points than (at least as many points as) $p$ and so $p$ would not be a winner (would not be a unique winner). We claim that $D^{\prime}$ corresponds to a vertex cover of $G$. For the sake of contradiction, assume that there is some edge $e_{j}$ incident to vertices $u$ and $v$ such that neither $u$ nor $v$ is in $D^{\prime}$. However, if this were the case then candidate $e_{j}$ would have more points than (at least as many points as) $p$ and so $p$ would not be a winner (would not be a unique winner). Thus, $D^{\prime}$ must form a vertex cover of size at most $k$.

Note that in the above proof it is crucial that $\alpha$ is neither 0 nor 1 . If $\alpha$ were 0 then the proof would fall apart because we would not be able to ensure that $D^{\prime}$ is a vertex cover, and if $\alpha$ were 1 then we would not be able to limit the size of $D^{\prime}$. In fact, we will now show, as Theorem 4.11, that both Copeland ${ }^{0}$ and Copeland ${ }^{1}$ are vulnerable to control via adding an unlimited number of candidates $\left(\mathrm{CCAC}_{\mathrm{u}}\right)$.

Theorem 4.11 Let $\alpha \in\{0,1\}$. Copeland ${ }^{\alpha}$ is vulnerable to constructive control via adding an unlimited number of candidates $\left(C C A C_{\mathrm{u}}\right)$, in both the nonunique-winner model and the unique-winner model, for both the rational and the irrational voter model.

Proof. Our input is candidate set $C$, spoiler candidate set $D$, a collection of voters with preferences (either preference lists or preference tables) over $C \cup D$, and a candidate $p \in C$. Our goal is to check whether there is some subset $D^{\prime} \subseteq D$ such that $p$ is a winner (the unique winner) of $\left(C \cup D^{\prime}, V\right)$ within Copeland ${ }^{\alpha}$. We will show that we can find such a set $D^{\prime}$, if it exists, by the following simple algorithm. 
Let $D_{1}=\left\{d \in D \mid \operatorname{score}_{(\{p, d\}, V)}^{\alpha}(p)=1\right\}$. Initialize $D^{\prime}$ to be $D_{1}$, and delete every $d \in D^{\prime}$ for which score ${ }_{\left(C \cup D^{\prime}, V\right)}^{\alpha}(p)<\operatorname{score}_{\left(C \cup D^{\prime}, V\right)}^{\alpha}(d)$. For the unique-winner problem, delete every $d \in D^{\prime}$ for which $\operatorname{score}_{\left(C \cup D^{\prime}, V\right)}^{\alpha}(p) \leq \operatorname{score}_{\left(C \cup D^{\prime}, V\right)}^{\alpha}(d)$.

Clearly, this algorithm runs in polynomial time. To show that the algorithm works, first note that for all $\widehat{D} \subseteq D$, if $p$ is a winner (the unique winner) of $(C \cup \widehat{D}, V)$, then $p$ is a winner (the unique winner) of $\left(C \cup\left(\widehat{D} \cap D_{1}\right), V\right)$. This is so because, by Observation 4.2,

$$
\begin{aligned}
\operatorname{score}_{(C \cup \widehat{D}, V)}^{\alpha}(p) & =\operatorname{score}_{\left(C \cup\left(\widehat{D} \cap D_{1}\right), V\right)}^{\alpha}(p)+\sum_{d \in \widehat{D}-D_{1}} \operatorname{score}_{(\{p, d\}, V)}^{\alpha}(p) \\
& =\operatorname{score}_{\left(C \cup\left(\widehat{D} \cap D_{1}\right), V\right)}^{\alpha}(p) .
\end{aligned}
$$

Now suppose that for some $\widehat{D} \subseteq D_{1}, p$ is a winner (the unique winner) of ( $\left.C \cup \widehat{D}, V\right)$, but that the algorithm computes a set $D^{\prime}$ such that $p$ is not a winner (not a unique winner) of $\left(C \cup D^{\prime}, V\right)$. We first consider the case that $\widehat{D} \subseteq D^{\prime}$. Since $p$ is not a winner (not a unique winner) of $\left(C \cup D^{\prime}, V\right)$, it follows by the construction of $D^{\prime}$ that there exists a candidate $d \in C-\{p\}$ such that $\operatorname{score}_{\left(C \cup D^{\prime}, V\right)}^{\alpha}(p)<$ $\operatorname{score}_{\left(C \cup D^{\prime}, V\right)}^{\alpha}(d)$ (such that $\operatorname{score}_{\left(C \cup D^{\prime}, V\right)}^{\alpha}(p) \leq \operatorname{score}_{\left(C \cup D^{\prime}, V\right)}^{\alpha}(d)$ ). However, in the nonunique-winner model we then have

$$
\begin{aligned}
\operatorname{score}_{\left(C \cup D^{\prime}, V\right)}^{\alpha}(p) & =\operatorname{score}_{(C \cup \widehat{D}, V)}^{\alpha}(p)+\left\|D^{\prime}\right\|-\|\widehat{D}\| \\
& \geq \operatorname{score}_{(C \cup \widehat{D}, V)}^{\alpha}(d)+\left\|D^{\prime}\right\|-\|\widehat{D}\| \geq \operatorname{score}_{\left(C \cup D^{\prime}, V\right)}^{\alpha}(d),
\end{aligned}
$$

which is a contradiction. In the unique-winner model, the first " $\geq$ " in the above inequality becomes a " $>$ " and we reach a contradiction as well.

Finally, consider the case that $\widehat{D} \nsubseteq D^{\prime}$. Let $d$ be the first candidate in $\widehat{D}$ that is deleted from $D^{\prime}$ in the algorithm. Then there is a set $D^{\prime \prime}$ such that $\widehat{D} \subseteq D^{\prime \prime} \subseteq D_{1}$ and $\operatorname{score}_{\left(C \cup D^{\prime \prime}, V\right)}^{\alpha}(p)<\operatorname{score}_{\left(C \cup D^{\prime \prime}, V\right)}^{\alpha}(d)$ in the nonunique-winner case $\operatorname{score}_{\left(C \cup D^{\prime \prime}, V\right)}^{\alpha}(p) \leq \operatorname{score}_{\left(C \cup D^{\prime \prime}, V\right)}^{\alpha}(d)$ in the unique-winner case). Since $\widehat{D} \subseteq D^{\prime \prime} \subseteq D_{1}$, we have

1. $\operatorname{score}_{(C \cup \widehat{D}, V)}^{\alpha}(p)=\operatorname{score}_{\left(C \cup D^{\prime \prime}, V\right)}^{\alpha}(p)-\left(\left\|D^{\prime \prime}\right\|-\|\widehat{D}\|\right)<\operatorname{score}_{\left(C \cup D^{\prime \prime}, V\right)}^{\alpha}(d)-\left(\left\|D^{\prime \prime}\right\|-\|\widehat{D}\|\right) \leq$ score $_{(C \cup \widehat{D}, V)}^{\alpha}(d)$ in the nonunique-winner case, and

2. $\operatorname{score}_{(C \cup \widehat{D}, V)}^{\alpha}(p)=\operatorname{score}_{\left(C \cup D^{\prime \prime}, V\right)}^{\alpha}(p)-\left(\left\|D^{\prime \prime}\right\|-\|\widehat{D}\|\right) \leq \operatorname{score}_{\left(C \cup D^{\prime \prime}, V\right)}^{\alpha}(d)-\left(\left\|D^{\prime \prime}\right\|-\|\widehat{D}\|\right) \leq$ $\operatorname{score}_{(C \cup \widehat{D}, V)}^{\alpha}(d)$ in the unique-winner case.

It follows that $p$ is not a winner (not a unique winner) of $(C \cup \widehat{D}, V)$. This is again a contradiction.

The remainder of this section is dedicated to showing that for any rational $\alpha$ such that $0 \leq \alpha \leq 1$, Copeland ${ }^{\alpha}$ is resistant to constructive control via deleting candidates and to constructive control via partitioning candidates (with or without run-off and in both the TE and the TP model). For reasons of space and nonrepetitiveness, the proofs of these results are not included here but can be found in the full TR version (Faliszewski et al., 2008b), where we first handle the case of constructive control via deleting candidates (CCDC) and then, using our proof for the CCDC case as a building block, handle the constructive partition-of-candidates cases. 
Theorem 4.12 Let $\alpha$ be a rational number such that $0 \leq \alpha \leq 1$. Copeland ${ }^{\alpha}$ is resistant to constructive control via deleting candidates $(C C D C)$, in both the nonunique-winner model and the unique-winner model, for both the rational and the irrational voter model.

The proof of Theorem 4.13 (which, as mentioned above, is presented in Faliszewski et al., 2008b) employs both the construction used for proving Theorem 4.12 and a construction that combines suitable elections such that the combined election has properties useful for proving various partition-of-candidates cases (with or without run-off). In particular, this construction not only is applied in the proof of Theorem 4.13, but also is designed to be general enough to serve as a key ingredient in proving Theorems 4.14, 4.15, and 4.16 below.

Theorem 4.13 Let $\alpha$ be a rational number such that $0 \leq \alpha \leq 1$. Copeland ${ }^{\alpha}$ is resistant to constructive control via run-off partition of candidates in both the ties-promote model (CCRPC-TP) and the ties-eliminate model (CCRPC-TE), in both the nonunique-winner model and the uniquewinner model, for both the rational and the irrational voter model.

Copeland $^{\alpha}$ is also resistant to constructive control via partition of candidates (without run-off) for each rational value of $\alpha$ between (and including) 0 and 1. However, the proofs for the TP and TE cases (which, again, can be found in the full TR version, Faliszewski et al., 2008b) are not as uniform as in the CCRPC scenario and so-to stay in sync with the structure of Faliszewski et al. 2008b, where the proofs are-we treat these cases separately as Theorems 4.14, 4.15, and 4.16.

Theorem 4.14 Let $\alpha$ be a rational number such that $0 \leq \alpha \leq 1$. Copeland ${ }^{\alpha}$ is resistant to constructive control via partition of candidates with the ties-promote tie-handling rule (CCPC-TP), in both the nonunique-winner model and the unique-winner model, for both the rational and the irrational voter model.

Theorem 4.15 Copeland ${ }^{1}$ is resistant to constructive control via partition of candidates with the ties-eliminate tie-handling rule (CCPC-TE), in both the nonunique-winner model and the uniquewinner model, for both the rational and the irrational voter model.

Theorem 4.16 Let $\alpha$ be a rational number, $0 \leq \alpha<1$. Copeland ${ }^{\alpha}$ is resistant to constructive control via partition of candidates with the ties-eliminate tie-handling rule (CCPC-TE), in both the nonunique-winner model and the unique-winner model, for both the rational and the irrational voter model.

\subsection{Voter Control}

In this section, we show that for each rational $\alpha, 0 \leq \alpha \leq 1$, Copeland $^{\alpha}$ is resistant to all types of voter control. Table 2 lists for each type of voter control, each rational $\alpha, 0 \leq \alpha \leq 1$, and each winner model (i.e., the nonunique-winner model and the unique-winner model) the theorem in which each given case is handled. We start with control via adding voters.

Theorem 4.17 Let $\alpha$ be a rational number such that $0 \leq \alpha \leq 1$. Copeland ${ }^{\alpha}$ is resistant to both constructive and destructive control via adding voters (CCAV and DCAV), in both the nonuniquewinner model and the unique-winner model, for both the rational and the irrational voter model. 


\begin{tabular}{|l|c|c|c|c|c|c|}
\hline \multirow{2}{*}{} & \multicolumn{2}{|c|}{$\alpha=0$} & \multicolumn{3}{c|}{$0<\alpha<1$} & \multicolumn{2}{c|}{$\alpha=1$} \\
\cline { 2 - 7 } & unique & nonunique & unique & nonunique & unique & nonunique \\
\hline CCAV & \multicolumn{5}{c|}{ Thm. 4.17 } \\
\hline DCAV & \multicolumn{7}{|c|}{ Thm. 4.21 } \\
\hline CCDV & Thm. 4.19 & Thm. 4.20 & Thm. 4.19 & Thm. 4.20 & Thm. 4.19 & Thm. 4.18 \\
\hline DCDV & Thm. 4.20 & Thm. 4.19 & Thm. 4.20 & Thm. 4.19 & Thm. 4.18 & Thm. 4.19 \\
\hline CCPV-TP & \multicolumn{7}{|c|}{ Thm-TP } & \multicolumn{7}{|c|}{ Thm. 4.23 } & Thm. 4.24 \\
\hline CCPV-TE & \multicolumn{7}{|c|}{ Thm. 4.26 } & Thm. 4.25 \\
\hline DCPV-TE & \multicolumn{7}{|c|}{}
\end{tabular}

Table 2: Table of theorems covering all resistance results for voter control for Copeland ${ }^{\alpha}$. Each theorem covers both the case of rational voters and the case of irrational voters.

Proof. Our result follows via reductions from the $\mathrm{X} 3 \mathrm{C}$ problem. We will first show how to handle the nonunique-winner constructive case and later we will argue that the construction can be easily modified for each of the remaining cases.

Let $(B, \mathscr{S})$ be an X3C instance where $B=\left\{b_{1}, \ldots, b_{3 k}\right\}$ and $\mathscr{S}=\left\{S_{1}, \ldots, S_{n}\right\}$ is a finite collection of three-element subsets of $B$. Without loss of generality, we assume that $k$ is odd (if it is even, we simply add $b_{3 k+1}, b_{3 k+2}, b_{3(k+1)}$ to $B$ and $S_{n+1}=\left\{b_{3 k+1}, b_{3 k+2}, b_{3(k+1)}\right\}$ to $\mathscr{S}$, and add 1 to $k$ ). The question is whether one can pick $k$ sets $S_{a_{1}}, \ldots, S_{a_{k}}$ such that $B=\bigcup_{j=1}^{k} S_{a_{j}}$.

We build a Copeland ${ }^{\alpha}$ election $E=(C, V)$ as follows. The candidate set $C$ contains candidates $p$ (the preferred candidate), $r$ ( $p$ 's rival), $s$, all members of $B$, and some number of padding candidates. We select the voter collection $V$ such that in their head-to-head contests, $s$ defeats $p, r$ defeats each $b_{i}$, and such that we have the following Copeland ${ }^{\alpha}$ scores for these candidates, where $\ell$ is some sufficiently large (but polynomially bounded in $n$ ) nonnegative integer:

1. $\operatorname{score}_{E}^{\alpha}(p)=\ell-1$,

2. $\operatorname{score}_{E}^{\alpha}(r)=\ell+3 k$, and

3. all other candidates have Copeland ${ }^{\alpha}$ scores below $\ell-1$.

It is easy to see that $E$ can be constructed in polynomial time by Lemma 4.8. In addition, we ensure that we have the following results of head-to-head contests between the candidates in $C$ :

1. $\operatorname{vs}_{E}(s, p)=k-1$,

2. for each $i \in\{1, \ldots, k\}, \operatorname{vs}_{E}\left(r, b_{i}\right)=k-3$, and

3. for all other pairs of candidates $c, d$, we have $\left|\operatorname{vs}_{E}(c, d)\right| \geq k+1$.

This can be done since we can add 2 to $\operatorname{vs}_{E}(c, d)$ and leave all other relative vote scores the same by adding two voters, $c>d>C-\{c, d\}$ and $\overleftarrow{C-\{c, d\}}>c>d$ (see Lemma 4.6). Since $k$ is odd and the number of voters is even (see Lemma 4.8), it is easy to see that we can fulfill these requirements. 
We also specify the set $W$ of voters that the chair can potentially add. For each set $S_{i} \in \mathscr{S}$ we have a single voter $w_{i} \in W$ with preference list

$$
p>B-S_{i}>r>S_{i}>\cdots
$$

(all unmentioned candidates follow in any fixed arbitrary order). We claim that $\mathscr{S}$ contains a $k$ element cover of $B$ if and only if $p$ can become a winner of the above election via adding at most $k$ voters selected from $W$.

If $\mathscr{S}$ contains a $k$-element cover of $B$, say $S_{a_{1}}, \ldots, S_{a_{k}}$, then we can make $p$ a winner via adding the voters from $U=\left\{w_{a_{1}}, \ldots, w_{a_{k}}\right\}$. Adding these $k$ voters increases $p$ 's score by one, since $p$ now defeats $s$ in their head-to-head contest. Since voters in $U$ correspond to a cover, the score of $r$ goes down by $3 k$ points. Why? For each $b_{i} \in B$, adding the $k-1$ voters in $U$ that correspond to the sets in the cover not containing $b_{i}$ increases the relative performance of $b_{i}$ versus $r$ by $k-1$ votes, thus giving $b_{i}$ two votes of advantage over $r$. Adding the remaining voter from $U$ decreases this advantage to 1 , but still $b_{i}$ wins the head-to-head contest with $r$.

We now show that if we can make $p$ a winner by adding at most $k$ voters then $\mathscr{S}$ contains a $k$ element cover of $B$. Note that $p$ is the only candidate that can possibly become a winner by adding at most $k$ voters, that $p$ can at best obtain Copeland ${ }^{\alpha}$ score $\ell$, that $p$ will obtain this score only if we add exactly $k$ voters, and that $r$ can lose at most $3 k$ points via losing his or her head-to-head contests with each of the $b_{i}$ 's. Thus the only way for $p$ to become a winner by adding at most $k$ voters from $W$ is that we add exactly $k$ voters such that $r$ loses his or her head-to-head contest with each $b_{i}$. Assume that $U \subseteq W$ is such a set of voters that does not correspond to a cover of $B$. This means that there is some candidate $b_{i}$ such that at least two voters in $U$ prefer $r$ to $b_{i}$. However, if this is the case then $b_{i}$ cannot defeat $r$ in their head-to-head contest and $p$ is not a winner. $U$ corresponds to a cover. This completes the proof of the nonunique-winner constructive case of the theorem.

For the constructive unique-winner case, we modify election $E$ so that $\operatorname{scor}_{E}^{\alpha}(p)=\ell$. All other listed properties of the relative vote scores and absolute Copeland ${ }^{\alpha}$ scores are unchanged. As in the previous case, it is easy to see that $p$ can become the unique winner via adding $k$ voters that correspond to a cover of $B$. For the converse, we will show that we still need to add exactly $k$ voters if $p$ is to become the unique winner.

If we added fewer than $k-1$ voters then $p$ would not get any extra points and so it would be impossible for $p$ to become the unique winner. Let us now show that adding exactly $k-1$ voters cannot make $p$ the unique winner. If we added exactly $k-1$ voters then $p$ would get $\alpha$ points extra from the tie with $s$. Now consider some candidate $b_{i} \in S_{j}$, where $S_{j}$ corresponds to one of the added voters, $w_{j}$. Since $w_{j}$ prefers $r$ to $b_{i}$, adding $w_{j}$ to the election increases the relative performance of $r$ versus $b_{i}$ to $k-2$. Thus adding the remaining $k-2$ voters can result in $b_{i}$ either tieing or losing his or her head-to-head contest with $r$. In either case $p$ would not have a high enough score to become the unique winner. Thus we know that exactly $k$ candidates must be added if we want $p$ to become the unique winner and, via the same argument as in the previous case, we know that they have to correspond to a cover.

For the destructive cases it suffices to note that the proof for the constructive nonunique-winner case works also as a proof for the destructive unique-winner case (where we are preventing $r$ from being the unique winner) and the constructive unique-winner case works also as a proof for the destructive nonunique-winner case (where we are preventing $r$ from being a winner).

Let us now turn to the case of control via deleting voters. Unfortunately, the proofs here are not as uniform as before and we need in some cases to handle $\alpha=1$ separately from the case where 
$0 \leq \alpha<1$. Also, we cannot use the construction lemma (Lemma 4.8) anymore to so conveniently build our elections. In the case of deleting voters (or partitioning voters) we need to have a very clear understanding of how each voter affects the election and the whole point of introducing the construction lemma was to abstract away from such low-level details.

Analogously to the case of candidate control, the resistance proofs for deleting voters are reused within the resistance proofs for partitioning voters. For reasons of space and nonrepetitiveness, we again do not include all proofs. In particular, the proofs of Theorems 4.18 and 4.20 are not included here but can be found in the full TR version (Faliszewski et al., 2008b). The proofs of Theorems 4.19 and 4.21, however, will be presented here. We mention that the construction given in the proof of Theorem 4.19 will be used later in the proof of Theorem 5.1, and the construction given in the proof of Theorem 4.21 will be used later in the proof of Theorem 5.2.

Theorem 4.18 Copeland ${ }^{1}$ is resistant to constructive control via deleting voters $(C C D V)$ in the nonunique-winner model and to destructive control via deleting voters (DCDV) in the uniquewinner model, for both the rational and the irrational voter model.

Theorem 4.19 Let $\alpha$ be a rational number such that $0 \leq \alpha \leq 1$. Copeland ${ }^{\alpha}$ is resistant to constructive control via deleting voters $(C C D V)$ in the unique-winner model and to destructive control via deleting voters $(D C D V)$ in the nonunique-winner model, for both the rational and the irrational voter model.

Proof. Let $(B, \mathscr{S})$ be an instance of X3C, where $B=\left\{b_{1}, \ldots, b_{3 k}\right\}$ and $\mathscr{S}=\left\{S_{1}, \ldots, S_{n}\right\}$ is a finite family of three-element subsets of $B$. Without loss of generality, we assume that $n \geq k$ and that $k>2$ (if $n<k$ then $\mathscr{S}$ does not contain a cover of $B$, and if $k \leq 2$ then we can solve the problem by brute force). We build an election $E=(C, V)$ such that:

1. If $\mathscr{S}$ contains a $k$-element cover of $B$, then the preferred candidate $p$ can become the unique Copeland $^{\alpha}$ winner of $E$ by deleting at most $k$ voters, and

2. if $r$ can become a nonwinner by deleting at most $k$ voters, then $\mathscr{S}$ contains a $k$-element cover of $B$.

Let the candidate set $C$ be $\left\{p, r, b_{1}, \ldots, b_{3 k}\right\}$ and let $V$ be the following collection of $4 n-k+1$ voters:

1. We have $n-1$ voters with preference $B>p>r$,

2. we have $n-k+2$ voters with preference $p>r>B$, and

3. for each $S_{i} \in \mathscr{S}$ we have two voters, $v_{i}$ and $v_{i}^{\prime}$, such that

(a) $v_{i}$ has preference $r>B-S_{i}>p>S_{i}$, and

(b) $v_{i}^{\prime}$ has preference $r>S_{i}>p>B-S_{i}$.

It is easy to see that for all $b_{i} \in B, \operatorname{vs}_{E}\left(r, b_{i}\right)=2 n-k+3, \operatorname{vs}_{E}\left(b_{i}, p\right)=k-3$, and $\operatorname{vs}_{E}(r, p)=k-1$.

If $\mathscr{S}$ contains a $k$-element cover of $B$, say $\left\{S_{a_{1}}, \ldots, S_{a_{k}}\right\}$, then we delete voters $v_{a_{1}}, \ldots, v_{a_{k}}$. In the resulting election, $p$ defeats every other candidate in their head-to-head contests, and thus $p$ is the unique winner. 
To prove the second statement, suppose that there is a subset $W$ of at most $k$ voters such that $r$ is not a winner of $\widehat{E}=(C, V-W)$. Since $\operatorname{vs}_{E}\left(r, b_{i}\right)=2 n-k+3$ and $n \geq k$, it is immediate that $r$ defeats every $b_{i} \in B$ in their head-to-head contests in $\widehat{E}$. In order for $r$ not to be a winner of $\widehat{E}$, $p$ must certainly defeat $r$ and tie-or-defeat every $b_{i} \in B$ in their head-to-head contests. But $p$ can defeat $r$ in their head-to-head contest only if $\|W\|=k$ and every voter in $W$ prefers $r$ to $p$. It follows that $W$ is a size- $k$ subset of $\left\{v_{1}, v_{1}^{\prime}, \ldots, v_{n}, v_{n}^{\prime}\right\}$.

Let $b_{i} \in B$. Recall that $\mathrm{vs}_{E}\left(b_{i}, p\right)=k-3$ and that $p$ needs to at least tie $b_{i}$ in their head-to-head contest in $\widehat{E}$. Since $\|W\|=k$, it follows that $W$ can contain at most one voter that prefers $p$ to $b_{i}$. Since $k>2$, it follows that $W$ contains only voters from the set $\left\{v_{1}, \ldots, v_{n}\right\}$ and that the voters in $W$ correspond to a $k$-element cover of $B$.

Theorem 4.20 Let $\alpha$ be a rational number such that $0 \leq \alpha<1$. Copeland ${ }^{\alpha}$ is resistant to constructive control via deleting voters $(C C D V)$ in the nonunique-winner model and to destructive control via deleting voters $(D C D V)$ in the unique-winner model, for both the rational and the irrational voter model.

Theorem 4.21 Let $\alpha$ be a rational number such that $0 \leq \alpha \leq 1$. Copeland ${ }^{\alpha}$ is resistant to both constructive and destructive control via partitioning voters in the TP model (CCPV-TP and DCPV$T P)$, in both the nonunique-winner model and the unique-winner model, for both the rational and the irrational voter model.

Proof. Let $(B, \mathscr{S})$ be an instance of X3C, where $B=\left\{b_{1}, \ldots, b_{3 k}\right\}$ and $\mathscr{S}=\left\{S_{1}, \ldots, S_{n}\right\}$ is a finite family of three-element subsets of $B$. Without loss of generality, we assume that $n \geq k$ and that $k>2$ (if $n<k$ then $S$ does not contain a cover of $B$, and if $k \leq 2$ then we can solve the problem by brute force). We build an election $E=(C, V)$ such that:

1. If $\mathscr{S}$ contains a $k$-element cover of $B$, then the preferred candidate $p$ can become the unique Copeland $^{\alpha}$ winner of $E$ via partitioning voters in the TP model, and

2. if $r$ can be made to not uniquely win $E$ via partitioning voters in the TP model, then $\mathscr{S}$ contains a $k$-element cover of $B$.

Note that this implies that Copeland ${ }^{\alpha}$ is resistant to both constructive and destructive control via partitioning voters in the TP model, in both the nonunique-winner model and the unique-winner model.

Our construction is an extension of the construction from Theorem 4.19. We let the candidate set $C$ be $\left\{p, r, s, b_{1}, \ldots, b_{3 k}\right\}$ and we let $V$ be the following collection of voters:

1. We have $k+1$ voters with preference $s>r>B>p$,

2. we have $n-1$ voters with preference $B>p>r>s$,

3. we have $n-k+2$ voters with preference $p>r>B>s$, and

4. for each $S_{i} \in \mathscr{S}$ we have two voters, $v_{i}$ and $v_{i}^{\prime}$, such that

(a) $v_{i}$ has preference $r>B-S_{i}>p>S_{i}>s$, and 
(b) $v_{i}^{\prime}$ has preference $r>S_{i}>p>B-S_{i}>s$.

Let $\widehat{V} \subseteq V$ be the collection of all the voters in $V$ except for the $k+1$ voters with preference $s>r>$ $B>p$. Note that $\widehat{V}$ is exactly the voter collection used in the proof of Theorem 4.19 with candidate $s$ added as the least desirable candidate. Since $s$ does not influence the differences between the scores of the other candidates, the following claim follows immediately from the proof of Theorem 4.19.

Claim 4.22 If $r$ can become a nonwinner of $(C, \widehat{V})$ by deleting at most $k$ voters, then $\mathscr{S}$ contains a $k$-element cover of $B$.

Recall that we need to prove that if $\mathscr{S}$ contains a $k$-element cover of $B$, then $p$ can be made the unique Copeland ${ }^{\alpha}$ winner of $E$ via partitioning voters in the TP model, and that if $r$ can be made to not uniquely win $E$ via partitioning voters in the TP model, then $\mathscr{S}$ contains a $k$-element cover of $B$.

If $\mathscr{S}$ contains a $k$-element cover of $B$, say $\left\{S_{a_{1}}, \ldots, S_{a_{k}}\right\}$, then we let the second subelection consist of the $k+1$ voters with preference $s>r>B>p$ and voters $v_{a_{1}}, \ldots, v_{a_{k}}$. Then $p$ is the unique winner of the first subelection, $s$ is the unique winner of the second subelection, and $p$ uniquely wins the final run-off between $p$ and $s$.

To prove the second statement, suppose there is a partition of voters such that $r$ is not a unique winner of the resulting election in model TP. Note that in at least one of the subelections, without loss of generality say the second subelection, a majority of the voters prefers $r$ to all candidates in $\left\{p, b_{1}, \ldots, b_{3 k}\right\}$. Since $r$ is the unique winner of every run-off he or she participates in, $r$ cannot be a winner of either subelection. Since $r$ defeats every candidate in $\left\{p, b_{1}, \ldots, b_{3 k}\right\}$ in their head-tohead contests in the second subelection, in order for $r$ not to be a winner of the second subelection, it must certainly be the case that $s$ defeats $r$ in their head-to-head contest in the second subelection. This implies that at most $k$ voters from $\widehat{V}$ can be part of the second subelection.

Now consider the first subelection. Note that $r$ cannot be a winner of the first subelection. Then, clearly, $r$ cannot be a winner of the first subelection restricted to voters in $\widehat{V} .{ }^{16}$ By Claim 4.22 it follows that $\mathscr{S}$ contains a $k$-element cover of $B$.

We now turn to the TE variant of control via partitioning voters. None of the remaining proofs of Section 4.2 (i.e., none of the proofs of Theorems 4.23 through 4.26) is included here but they each can be found in the full TR version (Faliszewski et al., 2008b). In particular, the proof of Theorem 4.23 uses the exact same construction as in the proof of Theorem 4.21 and the proofs of Theorems 4.24 and 4.25 use modifications thereof. To stay in sync with the structure of Faliszewski et al. 2008b, the proof-providing full TR (where the proof structure, as mentioned above, depends on the value of $\alpha$ ), we state each of Theorems 4.23 through 4.26 separately.

Theorem 4.23 Let $\alpha$ be a rational number such that $0 \leq \alpha<1$. Copeland ${ }^{\alpha}$ is resistant to constructive control via partitioning voters in the TE model (CCPV-TE), in both the nonunique-winner model and the unique-winner model, for both the rational and the irrational voter model.

16. If $r$ were a winner of the first subelection restricted to voters in $\widehat{V}$ then $r$ would certainly be a winner of the first subelection without any restrictions: The voters in $V-\widehat{V}$ prefer $r$ to everyone except $s$, and (by the discussion in the proof) $s$ cannot be a winner of the first subelection. (Note that $s$ can be a winner of at most one of the two subelections and $s$ is a winner of the second subelection.) 
Theorem 4.24 Copeland ${ }^{1}$ is resistant to constructive control via partitioning voters in the TE model (CCPV-TE), in both the nonunique-winner model and the unique-winner model, for both the rational and the irrational voter model.

Theorem 4.25 Copeland ${ }^{1}$ is resistant to destructive control via partitioning voters in the TE model (DCPV-TE), in both the nonunique-winner model and the unique winner model, for both the rational and the irrational voter model.

Finally, Theorem 4.26 states the resistance of Copeland ${ }^{\alpha}$, where $\alpha$ is a rational number with $0 \leq \alpha<1$, to destructive control by partition of voters in the TE model. The proof of this result (see Faliszewski et al., 2008b) extends the construction from the proof of Theorem 4.20 (see also Faliszewski et al., 2008b) in the same way the proof of Theorem 4.21 extended the construction from the proof of Theorem 4.19.

Theorem 4.26 Let $\alpha$ be a rational number such that $0 \leq \alpha<1$. Copeland ${ }^{\alpha}$ is resistant to destructive control via partitioning voters in the TE model (DCPV-TE), in both the nonunique-winner model and the unique-winner model, for both the rational and the irrational voter model.

\subsection{FPT Algorithm Schemes for Bounded-Case Control}

Resistance to control is generally viewed as a desirable property in system design. However, suppose one is trying to solve resistant control problems. Is there any hope?

Bartholdi, Tovey, and Trick (1989b), in their seminal paper on NP-hard winner-determination problems, suggested considering hard election problems for the cases of a bounded number of candidates or a bounded number of voters, and they obtained efficient-algorithm results for such cases. Within the study of elections, this same approach—seeking efficient fixed-parameter algorithmshas, for example, also been used (although somewhat tacitly—see the coming discussion in the second paragraph of Footnote 17) within the study of bribery (Faliszewski et al., 2006a; Faliszewski, Hemaspaandra, \& Hemaspaandra, 2006b). To the best of our knowledge, this bounded-case approach to finding the limits of resistance results has not been previously used to study control problems. In this section we do precisely that.

In particular, we obtain for resistant-in-general control problems a broad range of efficient algorithms for the case when the number of candidates or voters is bounded. Our algorithms are not merely polynomial time. Rather, we give algorithms that prove membership in FPT (fixedparameter tractability, i.e., the problem is not merely individually in $\mathrm{P}$ for each fixed value of the parameter of interest (voters or candidates), but indeed has a single $\mathrm{P}$ algorithm having degree that is bounded independently of the value of the fixed number of voters or candidates) when the number of candidates is bounded, and also when the number of voters is bounded. And we prove that our FPT claims hold even under the succinct input model-in which the voters are input via "(preference-list, binary-integer-giving-frequency-of-that-preference-list)" pairs-and even in the case of irrational voters. (One can imagine the succinct-representation case holding after some initial preprocessing of an election's ballots to compute the number of people casting each preference that occurred.)

We obtain such algorithms for all the voter-control cases, both for bounded candidates and for bounded voters, and for all the candidate-control cases with bounded candidates. On the other hand, we show that for the resistant-in-general irrational-voter, candidate-control cases, resistance still holds even if the number of voters is limited to being at most two. 
We structure this section as follows. We first start by briefly stating our notions and notations. We next state, and then prove, our fixed-parameter tractability results. Regarding those, we first address FPT results for the (standard) constructive and destructive cases. We then show that in many cases we can assert FPT results that are more general still-in particular, we will look at "extended control": completely pinpointing whether under a given type of control we can ensure that at least one of a specified collection of "Copeland Outcome Tables" (to be defined later) can be obtained. Finally, we give our resistance results.

\subsubsection{NOTIONS AND NOTATIONS}

The study of fixed-parameter complexity (see, e.g., Niedermeier, 2006) has been expanding explosively since it was parented as a field by Downey, Fellows, and others in the late 1980s and the 1990s. Although the area has built a rich variety of complexity classes regarding parameterized problems, for the purpose of the current paper we need focus only on one very important class, namely, the class FPT. Briefly put, a problem parameterized by some value $j$ is said to be fixed-parameter tractable (equivalently, to belong to the class FPT) if there is an algorithm for the problem whose running time is $f(j) n^{O(1)}$. (Note in particular that there is some particular constant for the "big-oh" that holds for all inputs, regardless of what $j$ value the particular input has.)

In our context, we will consider two parameterizations: bounding the number of candidates and bounding the number of voters. We will use the same notations used throughout this paper to describe problems, except we will postpend a "- $\mathrm{BV}_{j}$ " to a problem name to state that the number of voters may be at most $j$, and we will postpend a "- $\mathrm{BC}_{j}$ " to a problem name to state that the number of candidates may be at most $j$. In each case, the bound applies to the full number of such items involved in the problem. For example, in the case of control by adding voters, the $j$ must bound the total of the number of voters in the election added together with the number of voters in the pool of voters available for adding.

Typically, we have been viewing input votes as coming in each on a ballot. However, one can also consider the case of succinct inputs, in which our algorithm is given the votes as "(preferencelist, binary-integer-giving-frequency-of-that-preference-list)" pairs. (We mention in passing that for the "adding voter" cases, when we speak of succinctness we require that not just the always-voting voters be specified succinctly but also that the pool of voters-available-to-be-added be specified succinctly.) Succinct inputs have been studied extensively in the case of bribery (Faliszewski et al., 2006a, 2006b), and speaking more broadly, succinctness-of-input issues are often very germane to complexity classification (see, e.g., Wagner, 1986). Note that proving an FPT result for the succinct case of a problem immediately implies an FPT result for the same problem (without the requirement of succinct inputs being in place), and indeed is a stronger result, since succinctness can potentially exponentially compress the input.

Finally, we would like to be able to concisely express many results in a single statement. To do so, we borrow a notational approach from transformational grammar, and use square brackets as an "independent choice" notation. So, for example, the claim $\left[\begin{array}{c}\text { It } \\ \text { She } \\ \mathrm{He}\end{array}\right]\left[\begin{array}{c}\text { runs } \\ \text { walks }\end{array}\right]$ is a shorthand for six assertions: It runs; She runs; He runs; It walks; She walks; and He walks. A special case is the symbol " $\emptyset$ " which, when it appears in such a bracket, means that when unwound it should be viewed as no text at all. For example, "[ $\underset{\emptyset}{\operatorname{Succinct}}]$ Copeland is fun" asserts both "Succinct Copeland is fun" and "Copeland is fun." 


\subsubsection{Fixed-Parameter Tractability Results}

We immediately state our main results, which show that for all the voter-control cases FPT schemes hold for both the bounded-voter and bounded-candidate cases, and for all the candidate-control cases FPT schemes hold for the bounded-candidate cases.

Theorem 4.27 For each rational $\alpha, 0 \leq \alpha \leq 1$, and each choice from the independent choice brackets below, the specified parameterized (as $j$ varies over $\mathbb{N}$ ) problem is in FPT:

$$
\left[\begin{array}{c}
\text { succinct }^{\natural} \\
\emptyset
\end{array}\right]-\left[\begin{array}{c}
\text { Copeland }^{\alpha} \\
\text { Copeland }_{\text {Irrational }}^{\alpha}
\end{array}\right]-\left[\begin{array}{l}
\mathrm{C} \\
\mathrm{D}
\end{array}\right] \mathrm{C}\left[\begin{array}{c}
\mathrm{AV} \\
\mathrm{DV} \\
\mathrm{PV}-\mathrm{TE} \\
\mathrm{PV}-\mathrm{TP}
\end{array}\right]-\left[\begin{array}{c}
\mathrm{BV}_{j} \\
\mathrm{BC}_{j}
\end{array}\right] .
$$

Theorem 4.28 For each rational $\alpha, 0 \leq \alpha \leq 1$, and each choice from the independent choice brackets below, the specified parameterized (as $j$ varies over $\mathbb{N}$ ) problem is in FPT:

$$
\left[\begin{array}{c}
\text { succinct } \\
\emptyset
\end{array}\right]-\left[\begin{array}{c}
\text { Copeland }^{\alpha} \\
\text { Copeland }_{\text {Irrational }}^{\alpha}
\end{array}\right]-\left[\begin{array}{c}
\mathrm{C} \\
\mathrm{D}
\end{array}\right] \mathrm{C}\left[\begin{array}{c}
\mathrm{AC}_{\mathrm{u}} \\
\mathrm{AC} \\
\mathrm{DC} \\
\mathrm{PC}-\mathrm{TE} \\
\mathrm{PC}-\mathrm{TP} \\
\mathrm{RPC}-\mathrm{TE} \\
\mathrm{RPC}-\mathrm{TP}
\end{array}\right]-\mathrm{BC}_{j} .
$$

Readers not interested in a discussion of those results and their proofs can at this point safely skip to the next labeled section header.

Before proving the above theorems, let us first make a few observations about them. First, for cases where under a particular set of choices that same case is known (e.g., due to the results of Sections 4.1 and 4.2) to be in P even for the unbounded case, the above results are uninteresting as they follow from the earlier results (such cases do not include any of the "succinct" cases, since those were not treated earlier). However, that is a small minority of the cases. Also, for clarity as to what cases are covered, we have included some items that are not formally needed. For example, since FPT for the succinct case implies FPT for the no-succinctness-restriction case, and since FPT for the irrationality-allowed case implies FPT for the rational-only case, the first two choice brackets in each of the theorems could, without decreasing the results' strength, be removed by eliminating their " $\emptyset$ " and "Copeland ${ }^{\alpha}$ " choices.

We now turn to the proofs. Since proving every case would be uninterestingly repetitive, we will at times (after carefully warning the reader) prove the cases of one or two control types when that is enough to make clear how the omitted cases' proofs go.

Let us start with those cases that can be done simply by appropriately applied brute force.

We first prove Theorem 4.28.

Proof of Theorem 4.28. If we are limited to having at most $j$ candidates, then for each of the cases mentioned, the total number of ways of adding/deleting/partitioning candidates is simply a (large) constant. For example, there will be at most ("at most" rather than "exactly" since $j$ is merely an upper bound on the number of candidates) $2^{j}$ possible run-off partitions and there will be at most $2^{j-1}$ relevant ways of deleting candidates (since we can't (destructive case) or would 
never (constructive case) delete the distinguished candidate). So we can brute-force try all ways of adding/deleting/partitioning candidates, and for each such way can see whether we get the desired outcome. This works in polynomial time (with a fixed degree independent of $j$ and $\alpha$ ) even in the succinct case, and even with irrationality allowed.

- Theorem 4.28

A brute-force approach similarly works for the case of voter control when the number of voters is fixed. In particular, we prove the following subcase of Theorem 4.27.

Lemma 4.29 For each rational $\alpha, 0 \leq \alpha \leq 1$, and each choice from the independent choice brackets below, the specified parameterized (as $j$ varies over $\mathbb{N}$ ) problem is in $\mathrm{FPT}$ :

$$
\left[\begin{array}{c}
\text { succinct } \\
\emptyset
\end{array}\right]-\left[\begin{array}{c}
\text { Copeland }^{\alpha} \\
\text { Copeland }_{\text {Irrational }}^{\alpha}
\end{array}\right]-\left[\begin{array}{c}
\mathrm{C} \\
\mathrm{D}
\end{array}\right] \mathrm{C}\left[\begin{array}{c}
\mathrm{AV} \\
\mathrm{DV} \\
\mathrm{PV}-\mathrm{TE} \\
\mathrm{PV}-\mathrm{TP}
\end{array}\right]-\mathrm{BV}_{j} .
$$

When considering " $\mathrm{BV}_{j}$ " cases - namely in this proof and in the resistance section starting on page 334-we will not even discuss succinctness. The reason is that if the number of voters is bounded, say by $j$, then succinctness doesn't asymptotically change the input sizes interestingly, since succinctness at very best would compress the vote description by a factor of about $j$-which in this case is a fixed constant (relative to the value of the parameterization, which itself is $j$ ).

Proof of Lemma 4.29. If we are limited to having at most $j$ voters, note that we can, for each of these four types of control, brute-force check all possible approaches to that type of control. For example, for the case of control by deleting voters, we clearly have no more than $2^{j}$ possible vote deletion choices, and for the case of control by partitioning of voters, we again have at most $2^{j}$ partitions (into $V_{1}$ and $V-V_{1}$ ) to consider. And $2^{j}$ is just a (large) constant. So a direct brute-force check yields a polynomial-time algorithm, and by inspection one can see that its run-time's degree is bounded above independently of $j$.

Lemma 4.29

We now come to the interesting cluster of FPT cases: the voter-control cases when the number of candidates is bounded. Now, at first, one might think that we can handle this, just as the above cases, via a brute-force approach. And that is almost correct: One can get polynomial-time algorithms for these cases via a brute-force approach. However, for the succinct cases, the degrees of these algorithms will be huge, and will not be independent of the bound, $j$, on the number of candidates. For example, even in the rational case, one would from this approach obtain run-times with terms such as $n^{\|C\| !}$. That is, one would obtain a family of P-time algorithms, but one would not have an FPT algorithm.

To overcome this obstacle, we will employ Lenstra's (1983) algorithm for bounded-variablecardinality integer programming. Although Lenstra's algorithm is truly amazing in its power, even it will not be enough to accomplish our goal. Rather, we will use a scheme that involves a fixed (though very large) number of Lenstra-type programs each being focused on a different resolution path regarding the given problem.

What we need to prove, to complete the proof of Theorem 4.27, is the following lemma.

Lemma 4.30 For each rational $\alpha, 0 \leq \alpha \leq 1$, and each choice from the independent choice brackets below, the specified parameterized (as $j$ varies over $\mathbb{N}$ ) problem is in $\mathrm{FPT}$ : 


$$
\left[\begin{array}{c}
\text { succinct } \\
\emptyset
\end{array}\right]-\left[\begin{array}{c}
\text { Copeland }^{\alpha} \\
\text { Copeland }_{\text {Irrational }}^{\alpha}
\end{array}\right]-\left[\begin{array}{c}
\mathrm{C} \\
\mathrm{D}
\end{array}\right] \mathrm{C}\left[\begin{array}{c}
\mathrm{AV} \\
\mathrm{DV} \\
\mathrm{PV}-\mathrm{TE} \\
\mathrm{PV}-\mathrm{TP}
\end{array}\right]-\mathrm{BC}_{j}
$$

Let us start by recalling that, regarding the first choice bracket, the "succinct" case implies the " $\emptyset "$ " case, so we need only address the succinct case. Recall also that, regarding the second choice bracket, for each rational $\alpha, 0 \leq \alpha \leq 1$, the "Copeland Irrational " case implies the "Copeland $^{\alpha}$ " case, so we need only address the Copeland $\mathrm{Irrational}^{\alpha}$ case.

So all that remains is to handle each pair of choices from the third and forth choice brackets. To prove every case would be very repetitive. So we will simply prove in detail a difficult, relatively representative case, and then will for the other cases either mention the type of adjustment needed to obtain their proofs, or will simply leave it as a simple but tedious exercise that will be clear, as to how to do, to anyone who reads this section.

So, in particular, let us prove the following result.

Lemma 4.31 For each rational $\alpha, 0 \leq \alpha \leq 1$, the following parameterized (as $j$ varies over $\mathbb{N}$ ) problem is in FPT: succinct-Copeland Irrational $^{\alpha}$ CCPV-TP-BC ${ }_{j}$.

Proof. Let $\alpha, 0 \leq \alpha \leq 1$, be some arbitrary, fixed rational number. In particular, suppose that $\alpha$ can be expressed as $b / d$, where $b \in \mathbb{N}, d \in \mathbb{N}^{+}, b$ and $d$ share no common integer divisor greater than 1 , and if $b=0$ then $d=1$. We won't explicitly invoke $b$ and $d$ in our algorithm, but each time we speak of evaluating a certain set of pairwise outcomes "with respect to $\alpha$," one can think of it as evaluating that with respect to a strict pairwise win giving $d$ points, a pairwise tie giving $b$ points, and a strict pairwise loss giving 0 points.

We need a method of specifying the pairwise outcomes among a set of candidates. To do this, we will use the notion of a Copeland outcome table over a set of candidates. This will not actually be a table, but rather will be a function (a symmetric one-it will not be affected by the order of its two arguments) that, when given a pair of distinct candidates as inputs, will say which of the three possible outcomes allegedly happened: Either there is a tie, or one candidate won, or the other candidate won. Note that a COT is simply a representation of an election graph (see Section 4.1.2). So, in a $j$-candidate election, there are exactly $3^{\left(\begin{array}{l}j \\ 2\end{array}\right)}$ such functions. (We will not care about the names of the candidates, and so will assume that the tables simply use the names 1 through $j$, and that we match the names of the actual candidates with those integers by linking them lexicographically, i.e., the lexicographically first candidate will be associated with the integer 1 and so on.) Let us call a $j$-candidates Copeland outcome table a $j$-COT.

We need to build our algorithm that shows that the problem succinct-Copeland $_{\text {Irrational }}^{\alpha}$-CCPV-TP-BC $j, j \in \mathbb{N}$, is in FPT. So, let $j$ be some fixed integer bound on the number of candidates. ${ }^{17}$

17. We will now seem to specify the algorithm merely for this bound. However, it is important to note that we do enough to establish that there exists a single algorithm that fulfills the requirements of the definition of FPT. In particular, the specification we are about to give is sufficiently uniform that one can simply consider a single algorithm that, on a given input, notes the value of $j$, the number of candidates, and then does what the " $j$ " algorithm we are about to specify does.

We take this moment to mention in passing that our earlier work, Faliszewski et al. 2006a and (this is an expanded, full version of that) Faliszewski et al. 2006b, that gives P-time algorithms for the fixed parameter (fixed 
For each $j^{\prime}$-COT, $T_{1}$, For each $j^{\prime}$-COT, $T_{2}$,

Do

If

when we have a Copeland $\mathrm{d}_{\text {Irrational }}^{\alpha}$ election (involving all the input voters), with respect to $\alpha$, between all the candidates who win under $T_{1}$ with respect to $\alpha$, and all the candidates who win under $T_{2}$ with respect to $\alpha$, the preferred candidate of the input problem is a winner,

then

create and run the integer linear program constraint feasibility problem that checks whether there exists a partition of the voters such that the first subelection has $j^{\prime}$ COT $T_{1}$ and the second subelection has $j^{\prime}$-COT $T_{2}$, and if so, then accept.

Figure 5: The top-level code for the case succinct-Copeland Irrational $^{\alpha}$ CCPV-TP-BC ${ }_{j}$.

Let us suppose we are given an input instance. Let $j^{\prime} \leq j$ be the number of candidates in this instance (recall that $j$ is not the number of candidates, but rather is an upper bound on the number of candidates).

The top level of our algorithm is specified by the pseudocode in Figure 5. (Although this algorithm seemingly is just trying to tell whether the given control is possible for the given case, rather than telling how to partition to achieve that control, note that which iteration through the double loop accepts and the precise values of the variables inside the integer linear program constraint feasibility problem that made that iteration be satisfied will in fact tell us precisely what the partition is that makes the preferred candidate win.)

Now, note that the total number of $j^{\prime}$-COTs that exist (we do not need to care whether all can

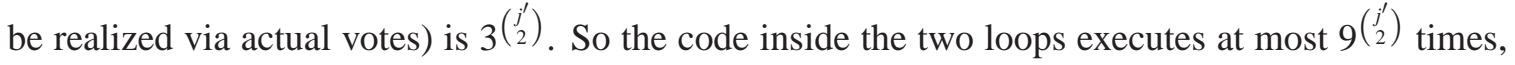
which is constant-bounded since $j^{\prime} \leq j$, and we have fixed $j$.

So all that remains is to give the integer linear program constraint feasibility problem mentioned inside the inner loop. The setting here can sometimes be confusing, e.g., when we speak of constants that can grow without limit. It is important to keep in mind that in this integer linear program constraint feasibility problem, the number of variables and constraints is constant (over all inputs), and the integer linear program constraint feasibility problem's "constants" (one may prefer the word

candidate and fixed voters) cases in fact, in all such claims we have in that work, implicitly is giving FPT algorithms, even though those papers don't explicitly note that. The reason is generally the same as why that is true in this paper-namely, the Lenstra technique is not just powerful but is also ideally suited for FPT algorithms and for being used inside algorithms that are FPT algorithms. Most interestingly, the Lenstra approach tends to work even on succinct inputs, and so the FPT comment we made applies even to those results in our abovementioned earlier papers that are about the succinct-inputs case of fixed-number-of-candidates and fixed-number-of-voters claims. (The fixednumber-of-candidates and fixed-number-of-voters Dodgson winner/score work of Bartholdi et al., 1989b, is known to be about FPT algorithms — due to the proof of Bartholdi et al., 1989b, itself, see the discussion in Faliszewski et al., 2006a, see also Betzler, Guo, and Niedermeier, 2008. Although the paper of Bartholdi et al., 1989b, doesn't address the succinct input model, Faliszewski et al., 2006a, notes that their approach works fine even in the succinct cases of the winner problem. That is true not just for the P-ness of their algorithms even in the succinct case, but also for the FPT-ness of their algorithms even in the succinct case.) 
coefficients, if that makes things clearer) are the only things that change with respect to the input. This is the framework that allows us to invoke Lenstra's powerful algorithm.

We first specify the set of constants of the integer linear program constraint feasibility problem.

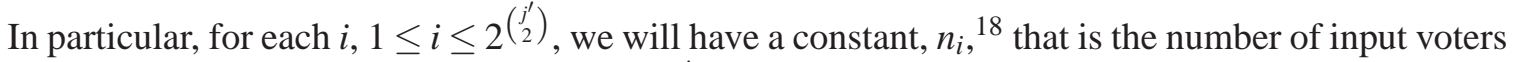

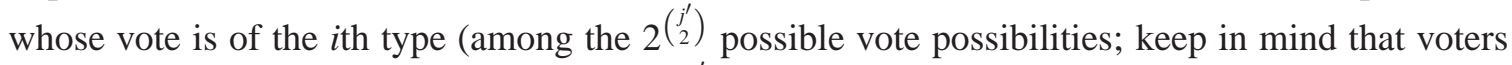
are allowed to be irrational, thus the value $2^{\left(\begin{array}{c}j^{\prime} \\ 2\end{array}\right)}$ is correct). Note that the number of these constants that we have is itself constant-bounded (for fixed $j$ ), though of course the values that these constants (of the integer linear program constraint feasibility problem) take on can grow without limit.

In addition, let us define some constants that will not vary with the input but rather are simply a notational shorthand that we will use to describe how the integer linear program constraint feasibility problem is defined (what constraints occur in it). In particular, for each $i$ and $\ell$ such that $1 \leq i \leq j^{\prime}$, $1 \leq \ell \leq j^{\prime}$, and $i \neq \ell$, let $v a l 1_{i, \ell}$ be 1 if $T_{1}$ asserts that (in their head-to-head contest) $i$ ties or defeats $\ell$, and let it be 0 if $T_{1}$ asserts that (in their head-to-head contest) $i$ loses to $\ell$. Let $v a l 2_{i, \ell}$ be identically defined, except with respect to $T_{2}$. Informally put, these values will be used to let our integer linear program constraint feasibility problem seek to enforce such a win/loss/tie pattern with respect to the given input vote numbers and the given type of allowed control action.

The integer linear program constraint feasibility problem's variables, which of course are all

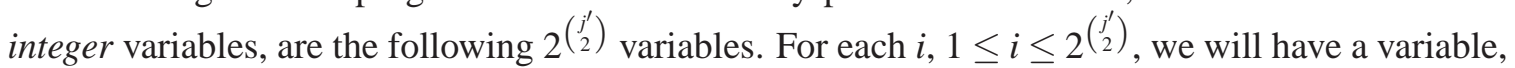
$m_{i}$, that represents how many of the $n_{i}$ voters having the $i$ th among the $2^{\left(\begin{array}{c}j^{\prime} \\ 2\end{array}\right)}$ possible vote types go into the first subelection.

Finally, we must specify the constraints of our integer linear program constraint feasibility problem. We will have three groups of constraints.

The first constraint group is enforcing that plausible numbers are put in the first partition. In particular, for each $i, 1 \leq i \leq 2^{\left(\begin{array}{c}j^{\prime} \\ 2\end{array}\right)}$, we have the constraints $0 \leq m_{i}$ and $m_{i} \leq n_{i}$.

The second constraint group is enforcing that after the partitioning we really do have in the first subelection a situation in which all the pairwise contests come out exactly as specified by $T_{1}$. In particular, for each $i$ and $\ell$ such that $1 \leq i \leq j^{\prime}, 1 \leq \ell \leq j^{\prime}$, and $i \neq \ell$, we do the following. Consider the equation

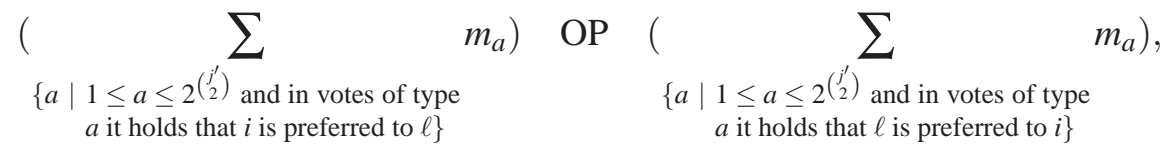

where $a$ in each sum varies over the $2^{\left(\begin{array}{c}j^{\prime} \\ 2\end{array}\right)}$ possible preferences. If $\operatorname{val} 1(i, \ell)=1$ we will have a constraint of the above form with OP set to " $\geq$ ". If $\operatorname{val} 1(\ell, i)=1$ we will have a constraint of the above form with OP set to " $\leq$ ". Note that this means that if $\operatorname{val} 1(i, \ell)=\operatorname{val} 1(\ell, i)=1$, i.e., those two voters are purported to tie, we will add two constraints.

The third constraint group has the same function as the second constraint group, except it regards the second subelection rather than the first subelection. In particular, for each $i$ and $\ell$ such that

18. Again, as discussed in the immediately previous paragraph, when we say that, for example, the $n_{i}$ are constants of the integer linear program constraint feasibility problem, we do not mean that they are constants in any complexity sense, but rather that they are the constants - in the sense of being the coefficients - of the integer linear program constraint feasibility problem. By saying that, we do not mean to imply that the number of voters is bounded by some global value over all cases. 
$1 \leq i \leq j^{\prime}, 1 \leq \ell \leq j^{\prime}$, and $i \neq \ell$, we do the following. Consider again equation (4.a) from above, except with each of the two occurrences of $m_{a}$ replaced by $n_{a}-m_{a}$. If val2 $(i, \ell)=1$ we will have a constraint of that form with OP set to " $\geq$ ". If $\operatorname{val} 2(\ell, i)=1$ we will have a constraint of that form with OP set to " $\leq$ ". As above, this means that if $\operatorname{val} 2(i, \ell)=\operatorname{val} 2(\ell, i)=1$, we will add two constraints.

This completes the specification of the integer linear programming constraint feasibility problem.

Note that our top-level code, from Figure 5, clearly runs within polynomial time relative to even the succinct-case input to the original CCPV-TP problem, and that that polynomial's degree is bounded above independently of $j$. Note in particular that our algorithm constructs at most a large constant (for $j$ fixed) number of integer linear programming constraint feasibility problems, and each of those is itself polynomial-sized relative to even the succinct-case input to the original CCPV-TP problem, and that polynomial size's degree is bounded above independently of $j$. Further, note that the integer linear programming constraint feasibility problems clearly do test what they are supposed to test-most importantly, they test that the subelections match the pairwise outcomes specified by $j^{\prime}$-COTs $T_{1}$ and $T_{2}$. Finally and crucially, by Lenstra's (1983) algorithm (see also Downey, 2003, and Niedermeier, 2002, which are very clear regarding the "linear"s later in this sentence), since this integer linear programming constraint feasibility problem has a fixed number of constraints (and in our case in fact also has a fixed number of variables), it can be solved-relative to its size (which includes the filled-in constants, such as our $n_{i}$ for example, which are in effect inputs to the integer program's specification) — via a linear number of arithmetic operations on linear-sized integers. So, overall, we are in polynomial time even relative to succinctly specified input, and the polynomial's degree is bounded above independently of $j$. Thus we have established membership in the class FPT.

We now describe very briefly how the above proof of Lemma 4.31 can be adjusted to handle all the partition cases from Lemma 4.30, namely, the cases $\left[\begin{array}{c}\text { succinct } \\ \emptyset\end{array}\right]-\left[\begin{array}{c}\text { Copeland }^{\alpha} \\ \text { Copeland }_{\text {Irrational }}^{\alpha}\end{array}\right]-\left[\begin{array}{l}\mathrm{C} \\ \mathrm{D}\end{array}\right] \mathrm{C}\left[\begin{array}{l}\mathrm{PV}-\mathrm{TE} \\ \mathrm{PV}-\mathrm{TP}\end{array}\right]-\mathrm{BC}_{j}$. As noted before, the first two brackets can be ignored, as we have chosen the more demanding choice for each. Let us discuss the other variations. Regarding changing from constructive to destructive, in Figure 5 change "is a winner" to "is not a winner." Regarding changing from PV-TP to PV-TE, in the "if" block in Figure 5 change each "all the candidates who win" to "the candidate who wins (if there is a unique candidate who wins)."

The only remaining cases are the cases $\left[\begin{array}{c}\text { succinct } \\ \emptyset\end{array}\right]-\left[\begin{array}{c}\text { Copeland }^{\alpha} \\ \text { Copeland }_{\text {Irrational }}^{\alpha}\end{array}\right]-\left[\begin{array}{l}\mathrm{C} \\ \mathrm{D}\end{array}\right] \mathrm{C}\left[\begin{array}{l}\mathrm{AV} \\ \mathrm{DV}\end{array}\right]-\mathrm{BC}_{j}$. However, these cases are even more straightforward than the partition cases we just covered, so for space reasons we will not write them out, but rather will briefly comment on these cases. Basically,

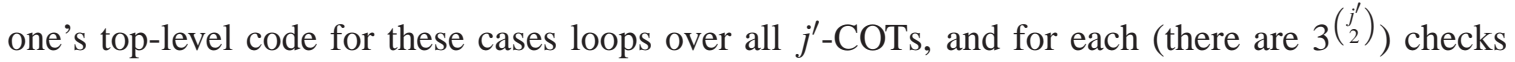
whether the right outcome happens under that $j^{\prime}$-COT (i.e., the distinguished candidate either is (constructive case) or is not (destructive case) a winner), and if so, it runs Lenstra's algorithm on an integer linear programming constraint feasibility problem to see whether we can by the allowed action (adding/deleting) get to a state where that particular $j^{\prime}$-COT matches our (after addition or deletion of voters) election. In the integer program, the variables will be the obvious ones, namely, for each $i, 1 \leq i \leq 2^{\left(\begin{array}{l}j^{\prime} \\ 2\end{array}\right)}$, we will have a variable, $m_{i}$, that describes how many voters of type $i$ to 
add/delete. As our key constants (of the integer linear program constraint feasibility problem), we will have, for each $i, 1 \leq i \leq 2^{\left(\begin{array}{l}i^{\prime} \\ 2\end{array}\right)}$, a value, $n_{i}$, for the number of type $i$ voters in the input. Also,

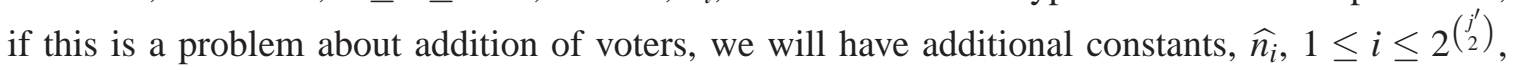
representing the number of type $i$ voters among the pool, $W$, of voters available for addition. And if our problem has an internal " $k$ " (a limit on the number of additions or deletions), we enforce that with the natural constraints, as do we also with the natural constraints enforce the obvious relationships between the $m_{i}, n_{i}, \widehat{n_{i}}$, and so on. Most critically, we have constraints ensuring that after the additions/deletions specified by the $m_{i}$, each pairwise outcome specified by the $j^{\prime}$-COT is realized.

Finally, although everything in Section 4.3 (both the part so far and the part to come) is written for the case of the nonunique-winner model, all the results hold analogously in the unique-winner model, with the natural, minor proof modifications. (Also, we mention in passing that due to the connection, found in Footnote 5 of Hemaspaandra et al., 2007a, between unique-winner destructive control and nonunique-winner constructive control, one could use some of our nonunique-winner constructive-case results to indirectly prove some of the unique-winner destructive-case results.)

\subsubsection{FPT AND EXTENDED CONTROL}

In this section, we look at extended control. By that we do not mean changing the ten standard control notions of adding/deleting/partitioning candidates/voters. Rather, we mean generalizing past merely looking at the constructive (make a distinguished candidate a winner) and the destructive (prevent a distinguished candidate from being a winner) cases. In particular, we are interested in control where the goal can be far more flexibly specified, for example (though in the partition cases we will be even more flexible than this), we will allow as our goal region any (reasonable - there are some time-related conditions) subcollection of "Copeland outcome tables" (specifications of who won/lost/tied each head-to-head contest). Since from a Copeland outcome table, in concert with the current $\alpha$, one can read off the Copeland $\mathrm{Irrational}^{\alpha}$ scores of the candidates, this allows us a tremendous range of descriptive flexibility in specifying our control goals, e.g., we can specify a linear order desired for the candidates with respect to their Copeland ${ }_{\text {Irrational }}^{\alpha}$ scores, we can specify a linear-order-with-ties desired for the candidates with respect to their Copeland ${ }_{\text {Irrational }}^{\alpha}$ scores, we can specify the exact desired Copeland $\alpha_{\text {Irrational }}^{\alpha}$ scores for one or more candidates, we can specify that we want to ensure that no candidate from a certain subgroup has a Copeland ${ }_{\text {Irrational }}^{\alpha}$ score that ties or defeats the Copeland $\mathrm{Irrational}^{\alpha}$ score of any candidate from a certain other subgroup, etc. ${ }^{19}$ Later in this section we will give a list repeating some of these examples and adding some new examples.

All the FPT algorithms given in the previous section regard, on their surface, the standard control problem, which tests whether a given candidate can be made a winner (constructive case) or can be precluded from being a winner (destructive case). We now note that the general approaches used in that section in fact yield FPT schemes even for the far more flexible notions of control mentioned

19. We mention up front that that initial example list applies with some additional minor technical caveats. Those examples were speaking as if in the final election we have all the candidates receiving Copeland ${ }_{\text {Irrational }}^{\alpha}$ scores in the final election. But in fact in the partition cases this is not (necessarily) so, and so in those cases we will focus on the Copeland outcome tables most natural to the given case. For example, in control by partition of voters, we will focus on subcollections of pairs of Copeland outcome tables for the two subelections. Also, though our Copeland outcome tables as defined below are not explicitly labeled with candidate names, but rather use a lexicographical correspondence with the involved candidates, in some cases we would - though we don't repeat this in the discussion below-need to allow the inclusion in the goal specification of the names of the candidates who are in play in a given table or tables, most particularly, in the cases of addition and deletion of candidates, and in some partition cases. 
above. In fact, one gets, for all the FPT cases covered in the previous section, FPT algorithms for the extended-control problem for those cases-very loosely put, FPT algorithms that test, for virtually any natural collection of outcome tables (as long as that collection itself can be recognized in a way that doesn't take too much running time, i.e., the checking time is polynomial and of a degree that is bounded independently of $j$ ), whether by the given type of control one can reach one of those outcome tables.

Let us discuss this in a bit more detail. A key concept used inside the proof of Lemma 4.31 was that of a Copeland outcome table - a function that for each distinct pair of candidates specifies either a tie or specifies who is the (not tied) winner in their pairwise contest. Let us consider the control algorithm given in the proof of that lemma, and in particular let us consider the top-level code specified in Figure 5. That code double-loops over size $j^{\prime}$ Copeland outcome tables (a.k.a. $j^{\prime}$ COTs), regarding the subpartitions, and for each case when the outcome tables' subelection cases, followed by the final election that they imply, correspond to the desired type of constructive (the distinguished person wins) or destructive (the distinguished person does not win) outcome, we check whether those two $j^{\prime}$-COTs can be made to hold via the current type of control (for the case being discussed, PV-TP).

However, note that simply by easily varying that top-level code we can obtain a natural FPT algorithm (a single algorithm, see Footnote 17 the analogue of which applies here) for any question of whether via the allowed type of control one can reach any run-time-quick-to-recognize collection of pairs of $j^{\prime}$-COTs (in the subelection), or even whether a given candidate collection and one of a given (run-time-quick-to-recognize) $j^{\prime \prime}$-COT collection over that candidate collection $\left(j^{\prime \prime}\right.$ being the size of that final-round candidate collection) can be reached in the final election. This is true not just for the partition cases (where, informally put, we would do this by, in Figure 5, changing the condition inside the "if" to instead look for membership in that collection of $j^{\prime}$-COTs ${ }^{20}$ ) but also for all the cases we attacked via Lenstra's method (though for the nonpartition cases we will typically single-loop over Copeland outcome tables that may represent the outcome after control is exerted; also, for some of these cases, the caveat at the end of Footnote 19 will apply). And it is even easier to notice that for those cases we attacked by direct brute force this also holds.

So, as just a few examples (some echoing the start of this section, and some new), all the following have (with the caveats mentioned above about needed names attached, e.g., in cases of candidate addition/deletion/partition, and regarding the partition cases focusing not necessarily directly on the

20. Let us discuss this a bit more formally, again using PV-TP as an example. Consider any family of boolean functions $F_{j}, j \in \mathbb{N}$, such that each $F_{j}$ is computable, even when its first argument is succinctly specified, in polynomial time with the polynomial degree bounded independently of $j$. Now, consider changing Figure 5's code to:

For each $j^{\prime}$-COT, $T_{1}$,

For each $j^{\prime}$-COT, $T_{2}$,

If $\left(F_{j^{\prime}}\left(\right.\right.$ input $\left.\left., T_{1}, T_{2}\right)\right)$

then $\cdots$.

Note that this change gives an FPT control scheme for a certain extended control problem. In particular, it does so for the extended control problem whose goal is to ensure that we can realize at least one of the set of $\left(T_{1}, T_{2}\right)$ such that $F_{j^{\prime}}\left(j^{\prime}\right.$ being the number of candidates in the particular input), given as its inputs the problem's input, $T_{1}$, and $T_{2}$ evaluates to true. That is, the $F_{j}$ functions are recognizing (viewed a bit differently, are defining) the goal set of the extended control problem.

From the input, $T_{1}$, and $T_{2}$ we can easily tell the scores in the final election. So this approach can be used to choose as our extended-control goals natural features of the final election. 
final table) FPT extended control algorithms for all the types of control and boundedness cases for which the FPT results of the previous section are stated.

1. Asking whether under the stated action one can obtain in the final election (simply in the election in the case when there is no partitioning) the outcome that all the Copeland Irrational $^{-}$ system scores of the candidates precisely match the relations of the lexicographic names of the candidates.

2. More generally than that, asking whether under the stated action one can obtain in the final election (simply in the election in the case when there is no partitioning) a certain linearorder-without-ties regarding the Copeland Irrational $^{\alpha}$-system scores of the candidates.

3. More generally still, asking whether under the stated action one can obtain in the final election (simply in the election in the case when there is no partitioning) a certain linear-order-with-

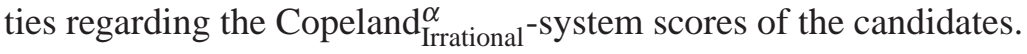

4. Asking whether under the stated action one can obtain in the final election (simply in the election in the case when there is no partitioning) the situation that exactly 1492 candidates tie as winner regarding their Copeland Irrational $^{\alpha}{ }^{\text {-system scores. }}$

5. Asking whether under the stated action one can obtain in the final election (simply in the election in the case when there is no partitioning) the situation that no two candidates have

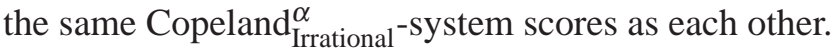

Again, these are just a very few examples. Our point is that the previous section is flexible enough to address not just constructive/destructive control, but also to address far more general control issues.

\subsubsection{RESistance RESUlts}

Theorems 4.27 and 4.28 give FPT schemes for all voter-control cases with bounded voters, for all voter-control cases with bounded candidates, and for all candidate-control cases with bounded candidates. This might lead one to hope that all the cases admit FPT schemes. However, the remaining type of case, the candidate-control cases with bounded voters, does not follow this pattern. In fact, we note that for Copeland ${ }_{\text {Irrational }}^{\alpha}$ all the candidate-control cases that we showed earlier in this paper (i.e., without bounds on the number of voters) to be resistant remain resistant even for the case of bounded voters. This resistance holds even when the input is not in succinct format, and so it certainly also holds when the input is in succinct format.

The reason for this is that, for the case of irrational voters, with just two voters (with preferences over $j$ candidates) any given $j$-COT can be achieved. To do this, for each distinct pair of candidates $i$ and $\ell$, to have $i$ preferred in their pairwise contest have both voters prefer $i$ to $\ell$, to have $\ell$ preferred in their pairwise contest have both voters prefer $\ell$ to $i$, and to have a tie in the pairwise contest have one voter prefer $\ell$ to $i$ and one voter prefer $i$ to $\ell$. Since in the proofs of resistance for candidate control, we identified elections with their election graphs, i.e., with their COTs, it is not hard to see that all these resistance proofs carry over even to the case of two irrational voters.

The only open cases remaining regard the rational-voter, candidate-control, bounded-voter cases. We note that Betzler and Uhlmann (2008) have recently resolved some of these open issues. 


\section{Control in Condorcet Elections}

In this section we show that Condorcet elections are resistant to constructive control via deleting voters (CCDV) and via partition of voters (CCPV). These results were originally claimed in the seminal paper of Bartholdi et al. (1992), but the proofs there were based on the assumption that a voter can be indifferent between several candidates. Their model of elections did not allow that (and neither does ours). Here we show how one can obtain these results in the case when the voters' preference lists are linear orders-which is both their model and ours.

Recall that a candidate $c$ of election $E=(C, V)$ is a Condorcet winner of $E$ if he or she defeats all other candidates in their head-to-head contests. Alternatively, one could say that a candidate $c$ is a Condorcet winner of election $E$ if and only if he or she has Copeland ${ }^{0}$ score of $\|C\|-1$. Since each election can have at most one Condorcet winner, it doesn't make sense here to differentiate between the unique-winner and the nonunique-winner models. (We pass on to the reader a referee's comment that in the very different system known as weak Condorcet elections, whose winners are all candidates who beat or tie each other candidate in head-to-head elections, one can have more than one winner.)

Theorem 5.1 Condorcet elections are resistant to constructive control via deleting voters.

Proof. This follows immediately from the proof of Theorem 4.19. Note that a Condorcet winner is always a unique Copeland ${ }^{\alpha}$ winner, for each rational $\alpha$ with $0 \leq \alpha \leq 1$, and note that in the proof of Theorem 4.19, if $\mathscr{S}$ contains a $k$-element cover of $B$, then we can delete $k$ voters such that in the resulting election $p$ defeats every other candidate in their head-to-head contest, i.e., $p$ is a Condorcet winner in the resulting election.

Before we proceed with our proof of resistance for the case of constructive control via partition of voters (CCPV), we have to mention a slight quirk of Bartholdi, Tovey, and Trick's model of voter partition. If one reads their paper carefully, it becomes apparent that they have a quiet assumption that each given set of voters can only be partitioned into subelections that each elect exactly one winner, thus severely restricting the chair's partitioning possibilities. That was why Hemaspaandra et al. (2007a) replaced Bartholdi, Tovey, and Trick's convention with the more natural ties-promote and ties-eliminate rules (see the discussion in Hemaspaandra et al., 2007a), but for this current section of our paper we go back to Bartholdi, Tovey, and Trick's model, since our goal here is to reprove their results without breaking their model.

Theorem 5.2 Condorcet elections are resistant to constructive control via partitioning voters $(C C P V)$ in Bartholdi, Tovey, and Trick's model (see the paragraph above).

Proof. The proof follows via a reduction from the $\mathrm{X} 3 \mathrm{C}$ problem. In fact, we use exactly the construction from the proof of Theorem 4.21. Let $E=(C, V)$ be the election constructed in that proof.

Since $s$ is the only candidate that $p$ defeats in a head-to-head contest, the only way for $p$ to become a winner via partitioning voters is to guarantee that $p$ wins within his or her subelection and that $s$ wins within the other one. (Note that since $p$ is not a Condorcet winner, $p$ cannot win in both subelections.)

If $\mathscr{S}$ contains a $k$-element cover, say, $\left\{S_{a_{1}}, \ldots, S_{a_{k}}\right\}$, then letting $V_{p}=\widehat{V}-\left\{v_{a_{1}}, \ldots, v_{a_{k}}\right\}$ and $V_{s}=V-V_{p}$ will make $p$ the Condorcet winner in this CCPV scenario. 
For the converse, let $\left(V_{p}, V_{s}\right)$ be a partition of the collection of voters such that $p$ is the global Condorcet winner in the CCPV scenario where we use two subelections, one with voters $V_{p}$ and one with voters $V_{s}$. Via the above paragraph we can assume, without loss of generality, that $p$ is the Condorcet winner in $\left(C, V_{p}\right)$ and that $s$ is the Condorcet winner in $\left(C, V_{s}\right)$. Since the $k+1$ voters in $V-\widehat{V}$ rank $s$ first and rank $p$ last, we can assume that $V_{s}$ contains these $k+1$ voters (i.e., the voters with preference $s>r>B>p$ ). Also, $V_{s}$ contains at most $k$ voters from $\widehat{V}$, as otherwise $s$ would certainly not be a Condorcet winner in $\left(C, V_{s}\right)$.

As a result, $p$ can be made the Condorcet winner of $(C, \widehat{V})$ by deleting at most $k$ voters. It follows from Claim 4.22 that $\mathscr{S}$ contains a $k$-element cover of $B$.

\section{Conclusions}

We have shown that from the computational point of view the election systems of Llull and Copeland (i.e., Copeland ${ }^{0.5}$ ) are broadly resistant to bribery and constructive procedural control, regardless of whether the voters are required to have rational preferences. It is rather charming that Llull's 700year-old system shows perfect resistance to bribery and more resistances to (constructive) control than any other natural system (even far more modern ones) with an easy winner-determination procedure-other than Copeland ${ }^{\alpha}, 0<\alpha<1$-is known to possess, and this is even more remarkable when one considers that Llull's system was defined long before control of elections was even explicitly studied. Copeland ${ }^{0.5}$ voting matches Llull's perfect resistance to bribery and in addition has perfect resistance to (constructive) control.

A natural open direction would be to study the complexity of control for additional election systems. It would be particularly interesting to find existing, natural voting systems that have polynomial-time winner determination procedures but that are resistant to all standard types of both constructive and destructive control. It would also be extremely interesting to find single results that classify, for broad families of election systems, precisely what it is that makes control easy or hard, i.e., to obtain dichotomy meta-results for control (see Hemaspaandra and Hemaspaandra, 2007, for some discussion regarding work of that flavor for manipulation).

\section{Acknowledgments}

We thank Nadja Betzler, Felix Brandt, Preetjot Singh, Frieder Stolzenburg, Dietrich Stoyan, the anonymous AAAI-07, AAIM-08, COMSOC-08, and JAIR referees, and JAIR handling editor Jeff Rosenschein for helpful comments, suggestions, and guidance. This work was supported in part by AGH-UST grant 11.11.120.777, DFG grants RO-1202/\{9-3, 11-1, 12-1\}, NSF grants CCR0311021, CCF-0426761, and IIS-0713061, the Alexander von Humboldt Foundation's TransCoop program, the European Science Foundation's EUROCORES program LogICCC, and Friedrich Wilhelm Bessel Research Awards to Edith Hemaspaandra and Lane A. Hemaspaandra. This work was done in part during visits by Piotr Faliszewski, Edith Hemaspaandra, and Lane A. Hemaspaandra to Heinrich-Heine-Universität Düsseldorf, during visits by Jörg Rothe to the University of Rochester, and while Piotr Faliszewski was at the University of Rochester. This paper combines and extends University of Rochester Computer Science Department Technical Reports TR-913 and TR-923, and some of this paper's results have been presented at the 22nd AAAI Conference on Artificial Intelligence (AAAI-07) in Faliszewski et al. 2007, at the October 2007 Dagstuhl Seminar on Computational Issues in Social Choice, at the 4th International Conference on Algorithmic Aspects 
in Information and Management (AAIM-08) in Faliszewski, Hemaspaandra, Hemaspaandra, and Rothe 2008a, and at the 2nd International Workshop on Computational Social Choice (COMSOC08).

\section{References}

Ahuja, R., Magnanti, T., \& Orlin, J. (1993). Network Flows: Theory, Algorithms, and Applications. Prentice-Hall.

Altman, A., \& Tennenholtz, M. (2007). An axiomatic approach to personalized ranking systems. In Proceedings of the 20th International Joint Conference on Artificial Intelligence, pp. 11871192. AAAI Press.

Arrow, K. (1951 (revised editon, 1963)). Social Choice and Individual Values. John Wiley and Sons.

Austen-Smith, D., \& Banks, J. (2000). Positive Political Theory I: Collective Preference. University of Michigan Press.

Bartholdi, III, J., \& Orlin, J. (1991). Single transferable vote resists strategic voting. Social Choice and Welfare, 8(4), 341-354.

Bartholdi, III, J., Tovey, C., \& Trick, M. (1989a). The computational difficulty of manipulating an election. Social Choice and Welfare, 6(3), 227-241.

Bartholdi, III, J., Tovey, C., \& Trick, M. (1989b). Voting schemes for which it can be difficult to tell who won the election. Social Choice and Welfare, 6(2), 157-165.

Bartholdi, III, J., Tovey, C., \& Trick, M. (1992). How hard is it to control an election? Mathematical and Computer Modeling, 16(8/9), 27-40.

Betzler, N., Guo, J., \& Niedermeier, R. (2008). Parameterized computational complexity of Dodgson and Young elections. In Proceedings of the 11th Scandinavian Workshop on Algorithm Theory, pp. 402-413. Springer-Verlag Lecture Notes in Computer Science \#5124.

Betzler, N., \& Uhlmann, J. (2008). Parameterized complexity of candidate control in elections and related digraph problems. In Proceedings of the 2nd Annual International Conference on Combinatorial Optimization and Applications, pp. 43-53. Springer-Verlag Lecture Notes in Computer Science \#5156.

Brams, S., \& Sanver, R. (2006). Critical strategies under approval voting: Who gets ruled in and ruled out. Electoral Studies, 25(2), 287-305.

Condorcet, J. (1785). Essai sur l'Application de L'Analyse à la Probabilité des Décisions Rendues à la Pluralité des Voix. Facsimile reprint of original published in Paris, 1972, by the Imprimerie Royale.

Conitzer, V., \& Sandholm, T. (2003). Universal voting protocol tweaks to make manipulation hard. In Proceedings of the 18th International Joint Conference on Artificial Intelligence, pp. 781788. Morgan Kaufmann.

Conitzer, V., \& Sandholm, T. (2006). Nonexistence of voting rules that are usually hard to manipulate. In Proceedings of the 21 st National Conference on Artificial Intelligence, pp. 627-634. AAAI Press. 
Conitzer, V., Sandholm, T., \& Lang, J. (2007). When are elections with few candidates hard to manipulate? Journal of the ACM, 54(3), Article 14.

Copeland, A. (1951). A "reasonable" social welfare function. Mimeographed notes from a Seminar on Applications of Mathematics to the Social Sciences, University of Michigan.

Cormen, T., Leiserson, C., Rivest, R., \& Stein, C. (2001). Introduction to Algorithms (second edition). MIT Press/McGraw Hill.

Downey, R. (2003). Parameterized complexity for the skeptic. In Proceedings of the 18th Annual IEEE Conference on Computational Complexity, pp. 147-168. IEEE Computer Society Press.

Duggan, J., \& Schwartz, T. (2000). Strategic manipulability without resoluteness or shared beliefs: Gibbard-Satterthwaite generalized. Social Choice and Welfare, 17(1), 85-93.

Dwork, C., Kumar, R., Naor, M., \& Sivakumar, D. (2001). Rank aggregation methods for the web. In Proceedings of the 10th International World Wide Web Conference, pp. 613-622. ACM Press.

Elkind, E., \& Lipmaa, H. (2005). Small coalitions cannot manipulate voting. In Proceedings of the 9th International Conference on Financial Cryptography and Data Security, pp. 285-297. Springer-Verlag Lecture Notes in Computer Science \#3570.

Ephrati, E., \& Rosenschein, J. (1997). A heuristic technique for multi-agent planning. Annals of Mathematics and Artificial Intelligence, 20(1-4), 13-67.

Erdélyi, G., Hemaspaandra, L., Rothe, J., \& Spakowski, H. (2007). On approximating optimal weighted lobbying, and frequency of correctness versus average-case polynomial time. In Proceedings of the 16th International Symposium on Fundamentals of Computation Theory, pp. 300-311. Springer-Verlag Lecture Notes in Computer Science \#4639.

Erdélyi, G., Nowak, M., \& Rothe, J. (2008a). Sincere-strategy preference-based approval voting broadly resists control. In Proceedings of the 33rd International Symposium on Mathematical Foundations of Computer Science, pp. 311-322. Springer-Verlag Lecture Notes in Computer Science \#5162.

Erdélyi, G., Nowak, M., \& Rothe, J. (2008b). Sincere-strategy preference-based approval voting fully resists constructive control and broadly resists destructive control. Tech. rep. arXiv:0806.0535 [cs.GT], arXiv.org. A precursor appears as (Erdélyi, Nowak, \& Rothe, 2008a). Journal version to appear in Mathematical Logic Quarterly.

Faliszewski, P. (2008). Nonuniform bribery (short paper). In Proceedings of the 7th International Conference on Autonomous Agents and Multiagent Systems, pp. 1569-1572. International Foundation for Autonomous Agents and Multiagent Systems.

Faliszewski, P., Hemaspaandra, E., \& Hemaspaandra, L. The complexity of bribery in elections. Journal of Artificial Intelligence Research. To appear.

Faliszewski, P., Hemaspaandra, E., \& Hemaspaandra, L. (2006a). The complexity of bribery in elections. In Proceedings of the 21st National Conference on Artificial Intelligence, pp. 641-646. AAAI Press. Journal version to appear as (Faliszewski, Hemaspaandra, \& Hemaspaandra, to appear). 
Faliszewski, P., Hemaspaandra, E., \& Hemaspaandra, L. (2006b). How hard is bribery in elections? Tech. rep. TR-895, Department of Computer Science, University of Rochester, Rochester, NY. Revised, September 2006.

Faliszewski, P., Hemaspaandra, E., Hemaspaandra, L., \& Rothe, J. (2007). Llull and Copeland voting broadly resist bribery and control. In Proceedings of the 22nd AAAI Conference on Artificial Intelligence, pp. 724-730. AAAI Press.

Faliszewski, P., Hemaspaandra, E., Hemaspaandra, L., \& Rothe, J. (2008a). Copeland voting fully resists constructive control. In Proceedings of the 4th International Conference on Algorithmic Aspects in Information and Management, pp. 165-176. Springer-Verlag Lecture Notes in Computer Science \#5034.

Faliszewski, P., Hemaspaandra, E., Hemaspaandra, L., \& Rothe, J. (2008b). Llull and Copeland voting computationally resist bribery and control. Tech. rep. arXiv:0809.4484 [cs.GT], Computing Research Repository, http://www.acm.org/repository/.

Faliszewski, P., Hemaspaandra, E., Hemaspaandra, L., \& Rothe, J. (2009). A richer understanding of the complexity of election systems. In Ravi, S., \& Shukla, S. (Eds.), Fundamental Problems in Computing: Essays in Honor of Professor Daniel J. Rosenkrantz, pp. 375-406. Springer.

Faliszewski, P., Hemaspaandra, E., \& Schnoor, H. (2008). Copeland voting: Ties matter. In Proceedings of the 7th International Conference on Autonomous Agents and Multiagent Systems, pp. 983-990. International Foundation for Autonomous Agents and Multiagent Systems.

Friedgut, E., Kalai, G., \& Nisan, N. (2008). Elections can be manipulated often. In Proceedings of the 49rd IEEE Symposium on Foundations of Computer Science, pp. 243-249. IEEE Computer Society.

Garey, M., \& Johnson, D. (1979). Computers and Intractability: A Guide to the Theory of NPCompleteness. W. H. Freeman and Company.

Ghosh, S., Mundhe, M., Hernandez, K., \& Sen, S. (1999). Voting for movies: The anatomy of recommender systems. In Proceedings of the 3rd Annual Conference on Autonomous Agents, pp. 434-435. ACM Press.

Gibbard, A. (1973). Manipulation of voting schemes. Econometrica, 41(4), 587-601.

Hägele, G., \& Pukelsheim, F. (2001). The electoral writings of Ramon Llull. Studia Lulliana, 41(97), 3-38.

Hemaspaandra, E., \& Hemaspaandra, L. (2007). Dichotomy for voting systems. Journal of Computer and System Sciences, 73(1), 73-83.

Hemaspaandra, E., Hemaspaandra, L., \& Rothe, J. (2007a). Anyone but him: The complexity of precluding an alternative. Artificial Intelligence, 171(5-6), 255-285.

Hemaspaandra, E., Hemaspaandra, L., \& Rothe, J. (2007b). Hybrid elections broaden complexitytheoretic resistance to control. In Proceedings of the 20th International Joint Conference on Artificial Intelligence, pp. 1308-1314. AAAI Press. Journal version to appear in Mathematical Logic Quarterly.

Homan, C., \& Hemaspaandra, L. Guarantees for the success frequency of an algorithm for finding Dodgson-election winners. Journal of Heuristics. To appear. Full version available as (Homan \& Hemaspaandra, 2005). 
Homan, C., \& Hemaspaandra, L. (2005). Guarantees for the success frequency of an algorithm for finding Dodgson-election winners. Tech. rep. TR-881, Department of Computer Science, University of Rochester, Rochester, NY. Revised, June 2007.

Kern, W., \& Paulusma, D. (2001). The new FIFA rules are hard: Complexity aspects of sports competitions. Discrete Applied Mathematics, 108(3), 317-323.

Lenstra, Jr., H. (1983). Integer programming with a fixed number of variables. Mathematics of Operations Research, 8(4), 538-548.

Levin, J., \& Nalebuff, B. (1995). An introduction to vote-counting schemes. The Journal of Economic Perspectives, 9(1), 3-26.

McCabe-Dansted, J., Pritchard, G., \& Slinko, A. (2008). Approximability of Dodgson's rule. Social Choice and Welfare, 31(2), 311-330.

McGarvey, D. (1953). A theorem on the construction of voting paradoxes. Econometrica, 21(4), 608-610.

McLean, I., \& Urken, A. (1995). Classics of Social Choice. University of Michigan Press.

Meir, R., Procaccia, A., Rosenschein, J., \& Zohar, A. (2008). The complexity of strategic behavior in multi-winner elections. Journal of Artificial Intelligence Research, 33, 149-178.

Merlin, V., \& Saari, D. (1997). Copeland method II: Manipulation, monotonicity, and paradoxes. Journal of Economic Theory, 72(1), 148-172.

Niedermeier, R. (2002). Invitation to fixed-parameter algorithms. Habilitation thesis, University of Tübingen.

Niedermeier, R. (2006). Invitation to Fixed-Parameter Algorithms. Oxford University Press.

Procaccia, A., \& Rosenschein, J. (2007). Junta distributions and the average-case complexity of manipulating elections. Journal of Artificial Intelligence Research, 28, 157-181.

Procaccia, A., Rosenschein, J., \& Kaminka, G. (2007). On the robustness of preference aggregation in noisy environments. In Proceedings of the 6th International Joint Conference on Autonomous Agents and Multiagent Systems, pp. 416-422. ACM Press.

Procaccia, A., Rosenschein, J., \& Zohar, A. (2008). On the complexity of achieving proportional representation. Social Choice and Welfare, 30(3), 353-362.

Saari, D., \& Merlin, V. (1996). The Copeland method I: Relationships and the dictionary. Economic Theory, 8(1), 51-76.

Satterthwaite, M. (1975). Strategy-proofness and Arrow's conditions: Existence and correspondence theorems for voting procedures and social welfare functions. Journal of Economic Theory, 10(2), 187-217.

Stearns, R. (1959). The voting problem. The American Mathematical Monthly, 66(9), 761-763.

Wagner, K. (1986). The complexity of combinatorial problems with succinct input representations. Acta Informatica, 23(3), 325-356.

Xia, L., \& Conitzer, V. (2008a). Generalized scoring rules and the frequency of coalitional manipulability. In Proceedings of the 9th ACM Conference on Electronic Commerce, pp. 109-118. ACM Press. 
Llull and Copeland Voting Resist Bribery And CONSTRUCTIVE Control

Xia, L., \& Conitzer, V. (2008b). A sufficient condition for voting rules to be frequently manipulable. In Proceedings of the 9th ACM Conference on Electronic Commerce, pp. 99-108. ACM Press.

Zermelo, E. (1929). Die Berechnung der Turnier-Ergebnisse als ein Maximumproblem der Wahrscheinlichkeitsrechnung. Mathematische Zeitschrift, 29(1), 436-460. 